\title{
Air quality and atmospheric deposition in the eastern US: 20 years of change
}

\author{
J. E. Sickles II ${ }^{1}$ and D. S. Shadwick ${ }^{2, \dagger}$ \\ ${ }^{1}$ Landscape Characterization Branch, Environmental Sciences Division, National Exposure \\ Research Laboratory, United States Environmental Protection Agency, Research Triangle Park, \\ North Carolina 27711, USA \\ ${ }^{2}$ Computer Sciences Corporation, Durham, North Carolina 27713, USA \\ $\dagger$ deceased \\ Correspondence to: J. E. Sickles II (sickles.joseph@epa.gov)
}

Received: 14 May 2014 - Published in Atmos. Chem. Phys. Discuss.: 3 July 2014

Revised: 17 October 2014 - Accepted: 22 October 2014 - Published: 9 January 2015

\begin{abstract}
Data collected in the eastern US between 1990 and 2009 at 34 paired dry and wet monitoring sites are examined. A goal is to evaluate the air quality impacts occurring between 1990 and 2009 that are associated with concurrent legislatively mandated changes in emissions. Four 5 -year periods, 1990-1994 (P1), 1995-1999 (P2), 2000-2004 (P3), and 2005-2009 (P4) are considered, with a primary focus on P1-to-P4 changes. Results suggest that legislatively mandated air pollution mitigation strategies have been successful in improving air quality and reducing atmospheric deposition in the eastern US.

Respective P1-to-P4 reductions of estimated sulfur dioxide $\left(\mathrm{SO}_{2}\right)$ and nitrogen oxides $\left(\mathrm{NO}_{\mathrm{x}}\right)$ emissions in the eastern US are 50 and $42 \%$. Corresponding behavior of the following metrics associated with these emissions reductions is examined: monitored atmospheric concentrations of $\mathrm{SO}_{2}$, aerosol sulfate $\left(\mathrm{SO}_{4}\right)$, and oxidized sulfur (S); dry, wet, and total deposition of $\mathrm{S}$; monitored atmospheric concentrations of nitric acid $\left(\mathrm{HNO}_{3}\right)$, aerosol nitrate $\left(\mathrm{NO}_{3}\right)$, and their sum, oxidized nitrogen $(\mathrm{OxN})$; dry, wet, and total deposition of $\mathrm{OxN}$; monitored atmospheric concentration of aerosol ammonium $\left(\mathrm{NH}_{4}\right)$; dry, wet, and total deposition of $\mathrm{NH}_{4}$; summed monitored atmospheric concentration of oxidized and reduced nitrogen $(\mathrm{N})$; dry, wet, and total deposition of $\mathrm{N}$; wet deposition of hydrogen ion $\left(\mathrm{H}^{+}\right)$; monitored atmospheric concentration of ozone $\left(\mathrm{O}_{3}\right)$; dry deposition of $\mathrm{O}_{3}$; and the summed monitored atmospheric concentration of aerosol $\mathrm{NO}_{3}, \mathrm{SO}_{4}$, and $\mathrm{NH}_{4}$ (Clean Air Status and Trends
\end{abstract}

Network particulate matter - CASTNET PM). Other metrics (e.g., ratios of dry to total deposition) are also considered.

Selected period-to-period changes of air quality and deposition metrics at site, regional, and seasonal scales are discussed. As an example, despite P1-to-P3 reductions in estimated emissions of both $\mathrm{SO}_{2}$ and $\mathrm{NO}_{\mathrm{x}}$, aerosol $\mathrm{NO}_{3}$ concentration increased in the east, with widespread wintertime numerical increases in both aerosol $\mathrm{NO}_{3}$ concentration and CASTNET PM. However, a reversal of this behavior is associated with continuing $\mathrm{P} 3$-to-P4 reductions of $\mathrm{SO}_{2}$ and $\mathrm{NO}_{\mathrm{x}}$ emissions. Thus, additional P3-to-P4 reductions of these emissions, especially $\mathrm{NO}_{\mathrm{x}}$, appear to have made progress in altering the chemical regime of the wintertime eastern US atmosphere so that future emissions reductions and their resulting reductions in aerosol concentrations may no longer be accompanied by sub-linear changes (or actual increases) in CASTNET PM.

\section{Introduction}

In the United States (US), pollution control legislation has been established to protect the public health and welfare by reducing emissions of selected pollutants. Both sulfur dioxide $\left(\mathrm{SO}_{2}\right)$ and nitrogen dioxide $\left(\mathrm{NO}_{2}\right)$ are designated by the US Environmental Protection Agency (EPA) as criteria pollutants, and they contribute to acidic deposition as well as airborne particulate matter (PM). In addition, nitrogen ox- 
Annual US $\mathrm{SO}_{2}$ and $\mathrm{NO}_{\mathrm{x}}$ Emissions

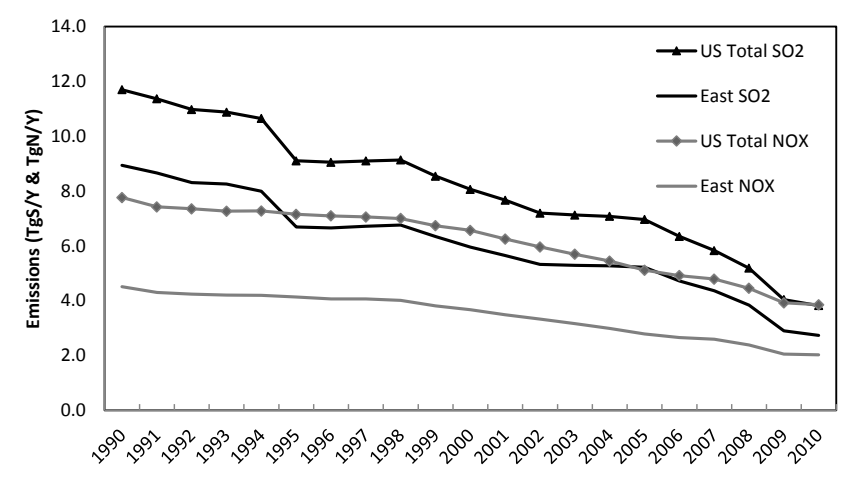

Figure 1. Annual US SO $2(\mathrm{TgS})$ and $\mathrm{NO}_{\mathrm{x}}(\mathrm{Tg} \mathrm{N})$ emissions estimates between 1990 and 2010 (Xing et al., 2013).

ides $\left(\mathrm{NO}_{\mathrm{x}}\right)$, the sum of nitric oxide $(\mathrm{NO})$ and $\mathrm{NO}_{2}$, act as precursors of another criteria pollutant, ozone $\left(\mathrm{O}_{3}\right)$.

Nationwide, in $1990,69 \%$ of $\mathrm{SO}_{2}$ emissions came from electric generating units (EGUs), whereas $82 \%$ of $\mathrm{NO}_{\mathrm{x}}$ emissions came from the sum of EGUs $(25 \%)$ and mobile sources (57\%) (Xing et al., 2013). Sources in the eastern states contributed 76 and $58 \%$ of the US total $1990 \mathrm{SO}_{2}$ and $\mathrm{NO}_{\mathrm{x}}$ emissions, respectively. The Clean Air Act Amendments of 1990 (CAAA) established phased year-round controls for $\mathrm{SO}_{2}$ and $\mathrm{NO}_{\mathrm{x}}$ emissions from EGUs that became effective in 1995 for $\mathrm{SO}_{2}$ and 1996 for $\mathrm{NO}_{\mathrm{x}}$ (Phase I), while additional controls became effective under Phase II in 2000. Over half of the EGUs targeted by the CAAA are located in six states along the Ohio River (source region - SR; Table 1). Beginning in 1999, $\mathrm{O}_{3}$ season (i.e., summertime) $\mathrm{NO}_{\mathrm{x}}$ controls focusing on EGUs became effective in selected eastern states under the Ozone Transport Commission (OTC) and were superceded in 2003 by the $\mathrm{NO}_{\mathrm{x}}$ State Implementation Plan (SIP) Call. The affected states chose to meet their mandatory reductions by participating in the $\mathrm{NO}_{\mathrm{x}}$ Budget Trading Program (BTP), a market-based cap and trade program for EGUs and large industrial units. The BTP was replaced in 2009 by the Clean Air Interstate Rule (CAIR). Various mobile source $\mathrm{NO}_{\mathrm{x}}$ emissions control programs also began in the mid- to late 1990s. Fully implemented in 2006, the Tier 2 Gasoline Sulfur Program targeted both $\mathrm{SO}_{2}$ and $\mathrm{NO}_{\mathrm{x}}$ emissions from mobile sources. These mobile source programs have a cumulative effect of reducing emissions over time as the mobile fleet is replaced.

Figure 1 shows estimates of emissions from 1990 to 2010 for both the total US and the eastern states (Xing et al., 2013). Both $\mathrm{SO}_{2}$ and $\mathrm{NO}_{\mathrm{x}}$ emissions show weak reductions between 1990 and 1994. There is a sharp reduction in $\mathrm{SO}_{2}$ emissions in 1995, when Phase I controls became effective. Although additional reductions of both $\mathrm{SO}_{2}$ and $\mathrm{NO}_{\mathrm{x}}$ emissions begin to appear in 1999, they are apparent in later years (e.g., after 2005 for $\mathrm{SO}_{2}$ ). Although a substantial portion of the long-term emissions reductions since 1990 has resulted

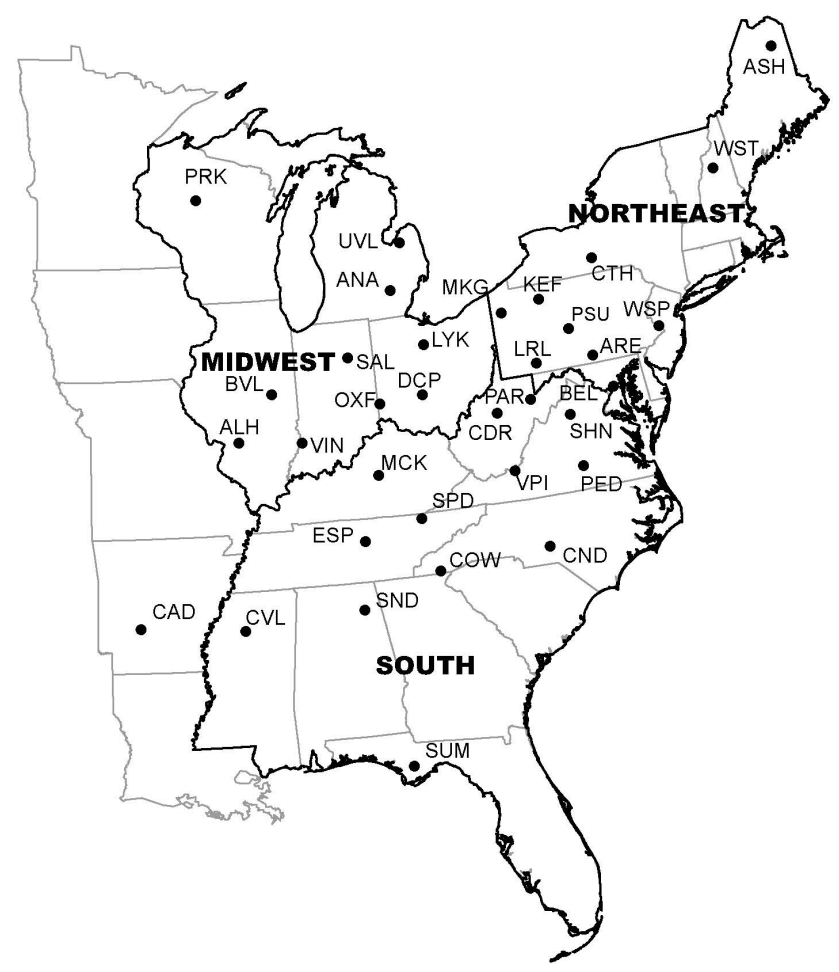

Figure 2. CASTNET monitoring sites and geographical regions considered in the current study (see Table 1).

from legislatively driven emissions controls, economic factors (e.g., coal-to-natural gas fuel switching and recessioninduced reduction in demand for electricity and transportation fuel) played a sizeable role between 2008 and 2009 (Lu et al., 2012).

A major objective of the current study is to examine the monitored and estimated air quality and deposition behaviors in the eastern US (E) between 1990 and 2009 associated with changes in emissions. The time period of interest is divided into four 5-year periods: 1990-1994 (period one, P1), 1995-1999 (period two, P2), 2000-2004 (period three, P3), and 2005-2009 (period four, P4). Focusing on these 5-year periods, average $\mathrm{P} 1$ US $\mathrm{SO}_{2}$ and $\mathrm{NO}_{\mathrm{x}}$ emissions have declined by 19 and $6 \%$ by P2, by 33 and $19 \%$ by P3, and by 49 and $37 \%$ by P4 (Xing et al., 2013). In the current study, the east is taken to represent all states east of the Mississippi River and is divided into three geographical regions: midwest (MW), northeast (NE), and south (SO) (Fig. 2). Mean period-specific emissions in these regions are illustrated for each 5-year period relative to P1 in Fig. 3.

This paper builds on the previous work (Sickles and Shadwick, 2007a and b; hereafter known as SS07a and SS07b). In SS07a seasonal and regional distributions of concentration and deposition of selected pollutants were examined for the 1990s (i.e., P1 and P2) at eastern rural US Clean Air Status and Trends Network (CASTNET) and paired National Atmospheric Deposition Program (NADP) monitoring sites. 
Table 1. Summary of characteristics of 34 CASTNET monitoring sites providing data in current study (see Fig. 2) listed by region.

\begin{tabular}{|c|c|c|c|c|c|c|c|c|}
\hline Site & Name & Land use $^{\mathrm{a}}$ & Terrain $^{b}$ & Elevation (m) & Region $^{\mathrm{d}}$ & $\begin{array}{l}\text { Special } \\
\text { category }\end{array}$ & $\begin{array}{l}\text { Latitude } \\
\left({ }^{\circ} \mathrm{N}\right)\end{array}$ & $\begin{array}{l}\text { Longitude } \\
\left({ }^{\circ} \mathrm{W}\right)\end{array}$ \\
\hline ALH & Alhambra, IL & A & FL & 164 & MW & SR & 38.9 & 89.6 \\
\hline ANA & Ann Arbor, MI & $\mathrm{F}$ & FL & 267 & MW & & 42.6 & 83.9 \\
\hline BVL & Bondville, IL & A & FL & 212 & MW & SR & 40.1 & 88.4 \\
\hline DCP & Deer Creek State Park, OH & A & $\mathrm{R}$ & 265 & MW & SR & 39.6 & 83.3 \\
\hline LYK & Lykens, OH & A & FL & 303 & MW & SR & 40.9 & 83.0 \\
\hline OXF & Oxford, $\mathrm{OH}$ & A & $\mathrm{R}^{\mathrm{c}}$ & 284 & MW & SR & 39.5 & 84.7 \\
\hline PRK & Perkinstown, WI & A & $\mathrm{R}$ & 472 & MW & $\mathrm{BKG}$ & 45.2 & 90.6 \\
\hline SAL & Salamonie Reservoir, IN & A & FL & 249 & MW & SR & 40.8 & 85.7 \\
\hline UVL & Unionville, MI & A & FL & 210 & MW & & 43.6 & 83.4 \\
\hline VIN & Vincennes, IN & A & $\mathrm{R}$ & 134 & MW & SR & 38.7 & 87.5 \\
\hline ARE & Arendtsville, PA & A & $\mathrm{R}$ & 269 & NE & SR & 39.9 & 77.3 \\
\hline ASH & Ashland, ME & A & FL & 235 & $\mathrm{NE}$ & BKG & 46.6 & 68.4 \\
\hline BEL & Beltsville, MD & S/A & FL & 46 & $\mathrm{NE}$ & & 39.0 & 76.8 \\
\hline CTH & Connecticut Hill, NY & $\mathrm{F}$ & $\mathrm{R}^{\mathrm{c}}$ & 515 & $\mathrm{NE}$ & HE & 42.4 & 76.7 \\
\hline KEF & Kane Experimental Forest, PA & $\mathrm{F}$ & $\mathrm{R}$ & 622 & $\mathrm{NE}$ & SR & 41.6 & 78.8 \\
\hline LRL & Laurel Hill State Park, PA & $\mathrm{F}$ & $\mathrm{C}^{\mathrm{c}}$ & 615 & $\mathrm{NE}$ & SR & 40.0 & 79.2 \\
\hline MKG & M.K. Goddard State Park, PA & $\mathrm{F}$ & $\mathrm{R}^{\mathrm{c}}$ & 384 & $\mathrm{NE}$ & SR & 41.4 & 80.2 \\
\hline PSU & Penn. State University, PA & A & $\mathrm{R}$ & 378 & $\mathrm{NE}$ & SR & 40.7 & 77.9 \\
\hline WSP & Washington's Crossing, NJ & $\mathrm{A} / \mathrm{S}$ & $\mathrm{R}$ & 58 & $\mathrm{NE}$ & & 40.3 & 74.9 \\
\hline WST & Woodstock, NH & $\mathrm{F}$ & $\mathrm{C}^{\mathrm{c}}$ & 258 & $\mathrm{NE}$ & & 43.9 & 71.7 \\
\hline CAD & Caddo Valley, AR & $\mathrm{F}$ & $\mathrm{R}^{\mathrm{c}}$ & 71 & SO & $\mathrm{BKG}$ & 34.2 & 93.1 \\
\hline CDR & Cedar Creek State Park, WV & $\mathrm{F}$ & $\mathrm{C}^{\mathrm{c}}$ & 234 & SO & SR & 38.9 & 80.9 \\
\hline CND & Candor, NC & $\mathrm{F}$ & $\mathrm{R}$ & 198 & SO & & 35.3 & 79.8 \\
\hline COW & Coweeta, NC & $\mathrm{F}$ & $\mathrm{C}^{\mathrm{c}}$ & 686 & SO & & 35.1 & 83.4 \\
\hline CVL & Coffeeville, MS & $\mathrm{F}$ & $\mathrm{R}$ & 134 & $\mathrm{SO}$ & & 34.0 & 89.8 \\
\hline ESP & Edgar Evins State Park, TN & $\mathrm{F}$ & $\mathrm{R}^{\mathrm{c}}$ & 302 & $\mathrm{SO}$ & & 36.0 & 85.7 \\
\hline MCK & Mackville, KY & A & $\mathrm{R}$ & 353 & SO & SR & 37.7 & 85.0 \\
\hline PAR & Parsons, WV & $\mathrm{F}$ & $\mathrm{C}^{\mathrm{c}}$ & 510 & SO & SR & 39.1 & 79.7 \\
\hline PED & Prince Edward, VA & $\mathrm{F}$ & $\mathrm{R}$ & 146 & SO & & 37.2 & 78.3 \\
\hline SHN & Big Meadows, VA & $\mathrm{F}$ & M & 1073 & $\mathrm{SO}$ & $\mathrm{HE}$ & 38.5 & 78.4 \\
\hline SND & Sand Mountain, AL & A & $\mathrm{R}$ & 352 & SO & & 34.3 & 86.0 \\
\hline SPD & Speedwell, TN & A & $\mathrm{R}$ & 361 & SO & & 36.5 & 83.8 \\
\hline SUM & Sumatra, FL & $\mathrm{F}$ & FL & 14 & SO & $\mathrm{BKG}$ & 30.1 & 85.0 \\
\hline VPI & Horton Station, VA & A & $M^{c}$ & 920 & $\mathrm{SO}$ & $\mathrm{HE}$ & 37.3 & 80.6 \\
\hline
\end{tabular}

In SS07b temporal changes in air quality and deposition in the eastern states between the early 1990s and early 2000s (i.e., P1-to-P3) were described and discussed. The record is expanded in the current paper to include a fourth 5-year period. Here, emphasis is on temporal changes occurring between the early 1990 s and late 2000 s (i.e., P1-to-P4) as new and continuing pollution controls have become mature and their impacts apparent. Results are displayed in a consistent manner to previous work (SS07a and b) to facilitate comparison. See Table 2 for definitions of terms and pollutant metrics used in the text, tables, and figures.

\section{Approach}

Monitoring data collected in the east between 1990 and 2009 at 34 CASTNET http://epa.gov/castnet/javaweb/index.html dry monitoring sites and paired NADP http://nadp.sws.uiuc. edu/ wet monitoring sites provide a sufficiently complete 20 -year record to permit examination. Weekly concentrations of gaseous $\mathrm{SO}_{2}$ and nitric acid $\left(\mathrm{HNO}_{3}\right)$, and aerosol sulfate $\left(\mathrm{SO}_{4}\right)$, nitrate $\left(\mathrm{NO}_{3}\right)$, and ammonium $\left(\mathrm{NH}_{4}\right)$ (using 3 -stage filter packs) and hourly concentrations of $\mathrm{O}_{3}$ (using continuous monitors) are determined at $10 \mathrm{~m}$. Dry deposition of airborne species is estimated using the infer- 
Table 2. Notation

\begin{tabular}{|c|c|}
\hline BKG & background \\
\hline $\mathrm{C}$ & concentration \\
\hline $\mathrm{CV}$ & coefficient of variation $(100 \mathrm{SD} /(\bar{x}))$ \\
\hline CASTNET PM & sum of aerosol $\mathrm{SO}_{4}, \mathrm{NO}_{3}$, and $\mathrm{NH}_{4}$ concentrations in total mass units \\
\hline $\mathrm{D}$ & deposition \\
\hline Dry $\mathrm{HNO}_{3}$ & dry deposition of monitored oxidized nitrogen from $\mathrm{HNO}_{3}$, computed as $\left(14 \times \mathrm{HNO}_{3}\right.$ deposition/63), in units of mass of nitrogen \\
\hline Dry $\mathrm{N}$ & dry deposition of nitrogen from $\mathrm{HNO}_{3}$, aerosol $\mathrm{NO}_{3}$, and aerosol $\mathrm{NH}_{4}$, computed as (Dry OxN + Dry $\left.\mathrm{NH}_{4}\right)$ \\
\hline Dry $\mathrm{NH}_{4}$ & dry deposition of monitored reduced nitrogen from aerosol $\mathrm{NH}_{4}$, computed as $\left(14 \times\right.$ dry $\mathrm{NH}_{4}^{+}$ion deposition/18), in units of mass of nitrogen \\
\hline Dry $\mathrm{NO}_{3}$ & dry deposition of monitored oxidized nitrogen from aerosol $\mathrm{NO}_{3}$, computed as $\left(14 \times\right.$ dry $\mathrm{NO}_{3}^{-}$ion deposition/62), in units of mass of nitrogen \\
\hline Dry $\mathrm{O}_{3}$ & dry deposition of ozone, in units of mass of $\mathrm{O}_{3}$ \\
\hline Dry OxN & dry deposition of monitored oxidized nitrogen from $\mathrm{HNO}_{3}$ and aerosol $\mathrm{NO}_{3}$, computed as $\left(\right.$ Dry $\left.\mathrm{HNO}_{3}+\mathrm{Dry}_{3}\right)$ \\
\hline Dry $S$ & dry deposition of oxidized sulfur from $\mathrm{SO}_{2}$ and aerosol $\mathrm{SO}_{4}$, computed as $\left(\right.$ Dry $\mathrm{SO}_{2}+$ Dry $\left.\mathrm{SO}_{4}\right)$ \\
\hline Dry $\mathrm{SO}_{2}$ & dry deposition of oxidized sulfur from $\mathrm{SO}_{2}$, computed as $\left(32 \times \mathrm{SO}_{2}\right.$ deposition/64), in units of mass of sulfur \\
\hline Dry $\mathrm{SO}_{4}$ & dry deposition of oxidized sulfur from aerosol $\mathrm{SO}_{4}$, computed as $\left(32 \times\right.$ dry $\mathrm{SO}_{4}^{2-}$ ion deposition/96), in units of mass of sulfur \\
\hline$(\mathrm{D} / \mathrm{T}) \mathrm{N}$ & ratio of dry $\mathrm{N}$ deposition to total $\mathrm{N}$ deposition, computed as Dry $\mathrm{N} /$ Total $\mathrm{N}$ \\
\hline$(\mathrm{D} / \mathrm{T}) \mathrm{NH}_{4}$ & ratio of dry $\mathrm{NH}_{4}$ deposition to total (dry plus wet) $\mathrm{NH}_{4}$ deposition, computed as Dry $\mathrm{NH}_{4} /$ Total $\mathrm{NH}_{4}$ \\
\hline$(\mathrm{D} / \mathrm{T}) \mathrm{OxN}$ & ratio of dry OxN deposition to total (dry plus wet) OxN deposition, computed as Dry OxN/Total OxN \\
\hline$(\mathrm{D} / \mathrm{T}) \mathrm{S}$ & ratio of dry $\mathrm{S}$ deposition to total (dry plus wet) $\mathrm{S}$ deposition, computed as Dry $\mathrm{S} /$ Total $\mathrm{S}$ \\
\hline $\mathrm{E}$ & eastern US \\
\hline $\mathrm{F}$ & fall (Sep, Oct, Nov) \\
\hline $\mathrm{HE}$ & high elevation \\
\hline $\mathrm{HNO}_{3}$ & nitric acid $\left(\mathrm{HNO}_{3}\right)$ concentration \\
\hline MW & midwest \\
\hline$n$ & number of site-specific period values comprising the mean \\
\hline $\mathrm{N}$ & monitored oxidized plus reduced nitrogen, present as $\mathrm{HNO}_{3}$, aerosol $\mathrm{NO}_{3}$ and aerosol $\mathrm{NH}_{4}$, in units of mass of nitrogen \\
\hline NE & northeast \\
\hline $\mathrm{NH}_{4}$ & ammonium $\left(\mathrm{NH}_{4}^{+}\right)$ion concentration \\
\hline NI & neutralization index, computed as $\left[\left(\mathrm{NH}_{4} / 18\right)-\left(\mathrm{NO}_{3} / 62\right)\right] / 2\left(\mathrm{SO}_{4} / 96\right)$, using airborne concentrations in molar units \\
\hline $\mathrm{NO}_{3}$ & nitrate $\left(\mathrm{NO}_{3}^{-}\right)$ion concentration \\
\hline $\mathrm{O}_{3}$ & ozone $\left(\mathrm{O}_{3}\right)$ concentration, in ppb \\
\hline OA & over all, i.e., period-site means averaged over sites $\approx$ annual average for $5 \mathrm{yr}$ period \\
\hline $\mathrm{OxN}$ & monitored oxidized nitrogen present as $\mathrm{HNO}_{3}$ and aerosol $\mathrm{NO}_{3}$, computed as $\left(14 \times \mathrm{HNO}_{3} / 63\right)+\left(14 \times \mathrm{NO}_{3} / 62\right)$, in units of mass of nitrogen \\
\hline PR & precipitation rate, in units of $\mathrm{cm} \mathrm{yr}^{-1}$ or $\mathrm{cm} \mathrm{season}^{-1}$ \\
\hline $\mathrm{RFHNO}_{3}$ & ratio of dry $\mathrm{HNO}_{3}$ deposition to dry OxN deposition, computed as Dry $\mathrm{HNO}_{3} /$ Dry OxN \\
\hline $\mathrm{RFSO}_{2}$ & ratio of dry $\mathrm{SO}_{2}$ deposition to dry $\mathrm{S}$ deposition, computed as Dry $\mathrm{SO}_{2} /$ Dry $\mathrm{S}$ \\
\hline $\mathrm{RHNO}_{3}$ & $\begin{array}{l}\text { ratio of oxidized nitrogen concentration from } \mathrm{HNO}_{3} \text { to oxidized nitrogen present as OxN, computed as } \\
\left(14 \times \mathrm{HNO}_{3} / 63\right) / \mathrm{OxN}\end{array}$ \\
\hline $\mathrm{RNFHNO}_{3}$ & ratio of dry $\mathrm{HNO}_{3}$ deposition to dry $\mathrm{N}$ deposition, computed as Dry $\mathrm{HNO}_{3} /$ Dry $\mathrm{N}$ \\
\hline $\mathrm{RNFNH}_{4}$ & ratio of dry $\mathrm{NH}_{4}$ deposition to dry $\mathrm{N}$ deposition, computed as Dry $\mathrm{NH}_{4} /$ Dry $\mathrm{N}$ \\
\hline $\mathrm{RNHNO}_{3}$ & $\begin{array}{l}\text { ratio of oxidized nitrogen concentration from } \mathrm{HNO}_{3} \text { to nitrogen present as } \mathrm{N} \text {, computed as } \\
\left(14 \times \mathrm{HNO}_{3} / 63\right) / \mathrm{N}\end{array}$ \\
\hline $\mathrm{RNNH}_{4}$ & $\begin{array}{l}\text { ratio of reduced nitrogen concentration from aerosol } \mathrm{NH}_{4} \text { to nitrogen present as } \mathrm{N} \text {, computed as } \\
\left(14 \times \mathrm{NH}_{4} / 18\right) / \mathrm{N}\end{array}$ \\
\hline $\mathrm{RNO}_{3}$ & $\begin{array}{l}\text { ratio of oxidized nitrogen concentration from aerosol } \mathrm{NO}_{3} \text { to oxidized nitrogen present as OxN, computed as } \\
\left(14 \times \mathrm{NO}_{3} / 62\right) / \mathrm{OxN} \text {, also as } 1-\mathrm{RHNO}_{3}\end{array}$ \\
\hline $\mathrm{RSO}_{2}$ & $\begin{array}{l}\text { ratio of airborne oxidized sulfur concentration from } \mathrm{SO}_{2} \text { to airborne oxidized sulfur present } \mathrm{S} \text {, computed as } \\
\left(32 \times \mathrm{SO}_{2} / 64\right) / \mathrm{S}\end{array}$ \\
\hline S & monitored oxidized sulfur present as $\mathrm{SO}_{2}$ and aerosol $\mathrm{SO}_{4}$, computed as $\left(32 \times \mathrm{SO}_{2} / 64\right)+\left(32 \times \mathrm{SO}_{4} / 96\right)$, in units of mass of sulfur \\
\hline SO & south \\
\hline $\mathrm{SO}_{2}$ & sulfur dioxide $\left(\mathrm{SO}_{2}\right)$ concentration \\
\hline $\mathrm{SO}_{4}$ & sulfate $\left(\mathrm{SO}_{4}^{2-}\right)$ ion concentration \\
\hline $\mathrm{Sp}$ & spring (Mar, Apr, May) \\
\hline SR & source region \\
\hline $\mathrm{Su}$ & summer (Jun, Jul, Aug) \\
\hline Total N & sum of total dry plus wet deposition of monitored nitrogen, computed as (Dry N + Wet N) \\
\hline Total $\mathrm{NH}_{4}$ & sum of dry plus wet deposition of monitored reduced nitrogen, computed as (Dry $\mathrm{NH}_{4}+$ Wet $\left.\mathrm{NH}_{4}\right)$, in units of mass of nitrogen \\
\hline Total OxN & sum of dry plus wet deposition of monitored oxidized nitrogen, computed as (Dry OxN + Wet OxN), in units of mass of nitrogen \\
\hline Total S & total dry plus wet oxidized sulfur deposition, computed as (Dry $S+$ Wet $S$ ), in units of mass of sulfur \\
\hline W & winter (Dec, Jan, Feb) \\
\hline Wet $\mathrm{H}^{+}$ & wet deposition of aqueous $\mathrm{H}^{+}$ion, in units of mass of hydrogen \\
\hline Wet $\mathrm{N}$ & wet deposition of nitrogen from aqueous $\mathrm{NO}_{3}^{-}$ion and aqueous $\mathrm{NH}_{4}^{+}$ion, computed as $\left(\right.$Wet $\left.\mathrm{OxN}+\mathrm{Wet} \mathrm{NH}_{4}\right)$ \\
\hline Wet $\mathrm{NH}_{4}$ & wet deposition of reduced nitrogen from aqueous $\mathrm{NH}_{4}^{+}$ion, computed as $\left(14 \times\right.$ aqueous $\mathrm{NH}_{4}^{+}$ion deposition/18), in units of mass of nitrogen \\
\hline Wet OxN & wet deposition of oxidized nitrogen from aqueous $\mathrm{NO}_{3}^{-}$ion, computed as $\left(14 \times\right.$ aqueous $\mathrm{NO}_{3}^{-}$ion deposition/62), in units of mass of nitrogen \\
\hline Wet $S$ & wet deposition of oxidized sulfur from aqueous $\mathrm{SO}_{4}^{2-}$ ion, computed as $\left(32 \times\right.$ aqueous $\mathrm{SO}_{4}^{2-}$ ion deposition/96), in units of mass of sulfur \\
\hline
\end{tabular}




\section{\% 1990-1994 Regional 5-Y Emissions}

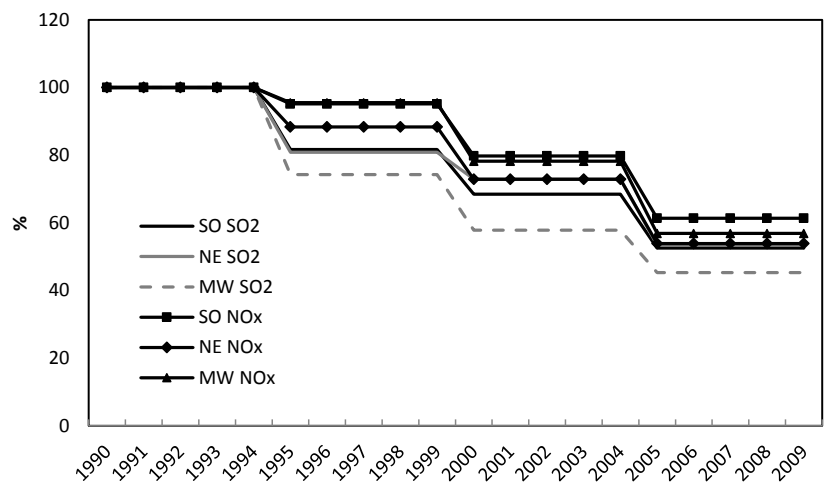

Figure 3. Estimates of regional mean annual $\mathrm{SO}_{2}$ and $\mathrm{NO}_{\mathrm{x}}$ emissions for four 5-year periods (P1, P2, P3, and P4) between 1990 and 2009, expressed as percentages of the 1990-1994 (P1) regional means. In the $\mathrm{SO}, \mathrm{NE}$, and $\mathrm{MW}$ mean $\mathrm{P} 1 \mathrm{SO}_{2}$ emissions are 3.48, 1.68, and $3.27 \mathrm{TgSyr}^{-1}$, and mean $\mathrm{P} 1 \mathrm{NO}_{\mathrm{x}}$ emissions are 1.89 , 1.06, and $1.35 \mathrm{Tg} \mathrm{Nyr}^{-1}$ (Xing et al., 2013).

ential model by multiplying modeled deposition velocities (Vd) by the monitored atmospheric concentration for the gas or aerosol species of interest. Deposition velocities for gases and aerosols are estimated using the multilayer model (MLM) (Meyers et al., 1998; Wesely et al., 1985). Monitoring data are composed from reported weekly (hourly for $\mathrm{O}_{3}$ ) measurements into estimates of means for year, site, and season. These year-site-season means, computed for atmospheric concentration, $\mathrm{Vd}$, precipitation rate (PR), and dry, wet, and total deposition, are averaged to period-site-season means and used to examine differences between 5-year periods for seasons, sites, and predefined groupings of sites. The data, methodologies, data quality (i.e., precision and accuracy), covariance of concentration and $\mathrm{Vd}$, impacts of nonideal landscape features, adjustments for sampling artifacts, and data completeness criteria for accepting mean estimates of measurement data are described in SS07a.

Monitoring site locations, 10 in the MW and NE, and 14 in the SO, are identified in Fig. 2 and Table 1. These 34 sites provide sparse coverage, especially in the southern part of the SO and the northeastern part of the NE. Three additional environmental groupings of monitoring sites are also defined as subsets of the eastern sites: background (BKG), high elevation (HE), and SR. These sites are chosen to reveal distinctions in pollutant behavior characteristic of their environments (Table 1 and SS07a).

CASTNET monitoring locations were initially sited to avoid the influence of major cities, highways, local activities, and point and area sources of pollutants and to be regionally representative (i.e., similar to a characteristic 80 by $80 \mathrm{~km}$ area surrounding the site) (Clarke et al., 1997; Baumgardner et al., 2002). In the current study, most of the CASTNET sites are located within $50 \mathrm{~km}$ of a paired NADP-NTN (National Trends Network) wet deposition site.

Collocated precision estimates (as CVs) of weekly CASTNET concentration, $\mathrm{Vd}$, and dry deposition at eastern sites are within 5 to $10 \%$ for the major species (Clarke et al., 1997; Sickles and Shadwick, 2002). Corresponding precision estimates are $5 \%$ for weekly NADP PR, wet S (i.e., wet deposition of sulfate; see Table 2), and wet $\mathrm{OxN} ; 10 \%$ for wet $\mathrm{H}^{+}$; and $15 \%$ for wet $\mathrm{NH}_{4}$ (Nilles et al., 1994). These estimates for weekly network results are likely to be conservative for corresponding longer-duration 5-year period results.

Total oxidized nitrogen $\left(\mathrm{NO}_{\mathrm{y}}\right)$ includes the primary $\mathrm{NO}_{\mathrm{x}}$ species ( $\mathrm{NO}$ and $\mathrm{NO}_{2}$ ) plus the secondary species $\left(\mathrm{HNO}_{3}\right.$, nitrous acid $\left(\mathrm{HNO}_{2}\right)$, aerosol $\mathrm{NO}_{3}$, peroxyacetylnitrate (PAN), as well as other oxidized organic nitrogen species). Total reduced nitrogen $\left(\mathrm{NH}_{\mathrm{x}}\right)$ includes both gaseous ammonia $\left(\mathrm{NH}_{3}\right)$ and aerosol $\mathrm{NH}_{4}$. In the current study, only two of the $\mathrm{NO}_{\mathrm{y}}$ species, $\mathrm{HNO}_{3}$ and aerosol $\mathrm{NO}_{3}$, (their sum is $\mathrm{OxN}$ ), and only one of the $\mathrm{NH}_{\mathrm{x}}$ species, aerosol $\mathrm{NH}_{4}$, are monitored. As a result, reported estimates of dry deposition of $\mathrm{OxN}$ and $\mathrm{NH}_{4}$ underestimate corresponding values for $\mathrm{NO}_{\mathrm{y}}$ and $\mathrm{NO}_{\mathrm{x}}$.

The contribution of coarse (i.e., $>2.5 \mu \mathrm{m}$ ) particles to total particulate deposition likely depends on location and proximity to sources of crustal dusts, road salt, and marine aerosols. Since the contribution of coarse particles is ignored in the MLM, where all particles are modeled as fine aerosol $\mathrm{SO}_{4}$ particles, current estimates of particulate dry deposition are biased low. However, the non-size selective CASTNET filter pack sampler likely over-samples the fine tail of coarse particle distribution. Although this may not be too important for aerosol $\mathrm{SO}_{4}$ and $\mathrm{NH}_{4}$, which occur mainly as fine particles, it would underestimate the contribution of dry deposition of aerosol $\mathrm{NO}_{3}$, where appreciable coarse aerosol $\mathrm{NO}_{3}$ can occur (Wolff, 1984; Lefer and Talbot, 2001).

As noted in SS07a (and references therein), use of the CASTNET filter pack sampler introduces sampling artifacts, especially to nitrogen-containing species. Findings from Sickles and Shadwick (2008), as well as wind tunnel and modeling studies (unpublished) of particle sampling characteristics of the non-size selective open-faced CASTNET filter pack inside its weather shield over a range of wind speeds, suggest appreciable collection of both fine $(<2.5 \mu \mathrm{m})$ and coarse particles on the first filter (Teflon) of the filter pack. Although both aerosol $\mathrm{SO}_{4}$ and $\mathrm{NH}_{4}$ exist mainly in the fine fraction $(<1 \mu \mathrm{m})$ year round, aerosol $\mathrm{NO}_{3}$ is multi-modal, and in non-marine environments exists mainly $(>75 \%)$ in the fine fraction in cool seasons and mainly as larger $(>4 \mu \mathrm{m})$ particles during warm seasons (Wolff, 1984; Lefer and Talbot, 2001; Zhang et al., 2008; Lee et al., 2008). Sampling artifacts in opposing directions may arise through possible loss of aerosol $\mathrm{NH}_{4} \mathrm{NO}_{3}$ during sampling and by aspiration of the fine tail of coarse aerosol $\mathrm{NO}_{3}$. Aerosol $\mathrm{NH}_{4} \mathrm{NO}_{3}$ (nominally $<2.5 \mu \mathrm{m}$ ) collected on the Teflon filter may be lost during sampling by volatilization into gaseous $\mathrm{HNO}_{3}$ and $\mathrm{NH}_{3}$, 
driven by departures from equilibrium (exaggerated at higher temperatures) and/or by reaction with strong acids. Results from comparison studies (Sickles and Shadwick, 2002, 2008; Lee et al., 2008; Zhang et al., 2009) are consistent with loss of $\mathrm{NH}_{4} \mathrm{NO}_{3}$ from the Teflon filter followed by collection of volatilized $\mathrm{HNO}_{3}$ on the downstream nylon filter of the CASTNET filter pack during warm season sampling and with minimal volatilization loss during cool season sampling. Coarse alkaline particles (e.g., sea/road salt or soil/mineral dust) may contain aerosol $\mathrm{NO}_{3}$ (possibly formed by scavenging $\mathrm{HNO}_{3}$ while airborne), and to the extent that they are collected, these larger particles may provide a high bias to CASTNET aerosol $\mathrm{NO}_{3}$ relative to its fine $\left(\mathrm{PM}_{2.5}\right)$ contribution. Some of these larger particles (if unreacted and retained on the Teflon filter) may scavenge airborne $\mathrm{HNO}_{3}$ from sampled air during week-long sampling periods, also providing a high bias to aerosol $\mathrm{NO}_{3}$ and a low bias to $\mathrm{HNO}_{3}$.

Some of these concepts are illustrated by the performance of Interagency Monitoring of Protected Visual Environments (IMPROVE) samplers with $2.5 \mu \mathrm{m}$ size selective inlet and CASTNET samplers collocated at 10 eastern monitoring sites (Sickles and Shadwick, 2008). At the eight non-marine sites good agreement was found for aerosol $\mathrm{SO}_{4}$ (CASTNET was biased high by 4 to $7 \%$ ), and more variability was found for aerosol $\mathrm{NO}_{3}$ (CASTNET ranged between $25 \%$ higher and $39 \%$ lower). Seasonally, aerosol $\mathrm{NO}_{3}$ showed median relative biases ranging between 5 and $-10 \%$ for all seasons, except summer, where the IMPROVE aerosol $\mathrm{NO}_{3}$ exceeded the corresponding CASTNET values by $>40 \%$ (this was at very low concentrations where the median bias was $0.07 \mu \mathrm{g} \mathrm{m}^{-3}$ ). Finally, although CASTNET and IMPROVE aerosol $\mathrm{NO}_{3}$ displayed mixed comparisons at eight inland sites, CASTNET sites showed consistently higher aerosol $\mathrm{NO}_{3}(40-100 \%)$ at the two marine-influenced sites, which was attributed to over-sampling the fine tail of coarse sea salt aerosols by the CASTNET sampler.

Thus, in the current study, minimal CASTNET sampling bias is expected for aerosol $\mathrm{SO}_{4}$ and $\mathrm{NH}_{4}$, which occur mainly as fine particles. However, $\mathrm{HNO}_{3}$ and aerosol $\mathrm{NO}_{3}$ as well as other metrics derived from them are subject to sampling biases that act in opposing directions. Volatilization of collected $\mathrm{NH}_{4} \mathrm{NO}_{3}$ during sampling could lead to a high bias of $\mathrm{HNO}_{3}$ and a low bias of aerosol $\mathrm{NO}_{3}$. In contrast, the nonsize selective sampler inlet could lead to an over-sampling of the fine tail of coarse aerosol $\mathrm{NO}_{3}$ with a high bias of aerosol $\mathrm{NO}_{3}$ and a potentially low bias of $\mathrm{HNO}_{3}$. The impacts of these biases are expected to be minimal during cool season sampling at non-marine sites. As a result, in the current study $\mathrm{HNO}_{3}$, aerosol $\mathrm{NO}_{3}$, and metrics derived from them (e.g., CASTNET PM, Dry $\mathrm{HNO}_{3}$, Dry $\mathrm{NO}_{3}, \mathrm{RNO}_{3}$ and other ratios of concentration and deposition) should be viewed with caution, especially during summer.

Uncertainties in Vd, rather than concentration, likely limit the accuracy of dry deposition estimates (Hicks et al., 1991; Lovett and Lindberg, 1993). Accuracy of Vd estimates has not been well established. Based on comparisons with field measurements, the MLM may provide fairly accurate estimates of $\mathrm{Vd}$ for $\mathrm{SO}_{2}, \mathrm{O}_{3}$, and $\mathrm{HNO}_{3}$ to many surfaces (e.g., crops; Meyers et al., 1998); however, $\mathrm{Vd}$ for $\mathrm{SO}_{2}$ to forested canopies may be underestimated by $\approx 35 \%$ (Finkelstein et al., 2000). Recent comparison at the same site of weekly CASTNET MLM Vd estimates with those from a similar model used by the Canadian Air and Precipitation Monitoring Network (CAPMoN) shows CASTNET Vd to be low (relative to CAPMoN) by approximately $50 \%$ for $\mathrm{SO}_{2}$ and $35 \%$ for $\mathrm{HNO}_{3}$; differences of hourly estimates between the two models could be as large as a factor of 2-3 (Schwede et al., 2011). Annual average small-scale spatial variability $(0.5 \mathrm{~km})$ of model-estimated $\mathrm{Vd}$ was estimated at $10 \%$ for $\mathrm{SO}_{2}$ and $\mathrm{O}_{3}, 30 \%$ for aerosol $\mathrm{SO}_{4}$, and $40 \%$ for $\mathrm{HNO}_{3}$ (Brook et al., 1997). Uncertainty of the inferential model has been estimated at $25 \%$ for $\mathrm{O}_{3}, 30 \%$ for $\mathrm{SO}_{2}$ and $\geq 40 \%$ for $\mathrm{HNO}_{3}$ and particles (Clarke et al., 1997). These estimates may be appropriate for areas with ideal terrain and uniform land use; however, non-ideal landscape features (e.g., irregular terrain and/or forested canopies) likely contribute low biases to concentration, estimated $\mathrm{Vd}$, and dry deposition estimates, and increase their resulting uncertainty (Clarke et al., 1997; Hicks, 2006). The distribution of influential landscape features across sites suggests that their impacts result in the greatest underestimates at sites in the south and northeast geographical regions, and that their impacts should be smaller at sites in the midwestern grouping. Much of the above evidence suggests that CASTNET Vd estimates, while uncertain, likely provide conservative lower bound estimates of dry deposition.

Internetwork comparisons of annual wet deposition have been conducted for the periods 1986-1993 (Sirois et al., 2000) and 1995-2004 (Wetherbee et al., 2010) at collocated NADP and CAPMoN sites. NADP results for precipitation depth and wet deposition of $\mathrm{H}^{+}, \mathrm{NH}_{4}, \mathrm{~S}$, and $\mathrm{OxN}$ appear to be biased low relative to CAPMoN by less than $30 \%$. Although there are no standards for determining accuracy, current NADP results are possibly biased low and likely accurate within $25 \%$.

The east has large heterogeneities in its spatial distribution of land cover (i.e., crops, forests, and suburban), of terrain (i.e., flat, rolling, complex, and montane) and of pollution sources (e.g., EGU sources in the Ohio Valley and mobile sources in urban centers). As a result, it is unlikely that the sparse coverage of CASTNET (i.e., 34 sites) can provide truly accurate regional representations. This is supported by a recent modeling study of wet deposition (Sickles et al., 2009) where current study site locations were found to over represent geographical areas subject to both high emissions and wet deposition, yielding over-estimations of corresponding regional values by 5 to $25 \%$. Nevertheless, for the current study, values representing areas with common geographical and environmental characteristics are approximated as the means of values from sites associated with each classifica- 
tion. It is assumed that these estimates are useful as rough indices of their respective geographical regions and environmental groupings.

As indicated above, the airborne concentration, estimated $\mathrm{Vd}$, and dry and wet deposition estimates may be subject to sizeable uncertainties. However, assuming constant bias, their precision to a first approximation is expected to control the uncertainty associated with the current examination of period-to-period (e.g., P1-to-P4) temporal changes.

Data have been composed from reported measurements into estimates of means for year, site, and season. Yearsite-season means were computed for atmospheric concentration, Vd, PR, and dry, wet, and total deposition. Consistent with previous work (SS07a and b) these values, averaged to period-site-season means, are used to examine differences between 5-year emission periods for seasons, sites, and predefined site groupings. Seasons (e.g., data for winter, W, 1990 include data from December 1989 through February 1990; spring, Sp; summer, Su; and fall, F) are used to define the years in periods. Five-year periods have been chosen to coincide with emission patterns (noted earlier), to minimize the impacts of missing data on seasonal means, to reduce the impact of meteorological variability, and to maintain consistency with earlier studies. For example, Table 3 presents averages of year-site-season means averaged over all the years in $\mathrm{P} 4$ and all the sites in each specified site grouping. Analogous tabulations for P1, P2, and P3 may be found in SS07a and $b$.

The approach to data analysis, described in detail in SS07b, uses mean squared error derived from analysis of variance of mean estimates for each pollutant metric to examine period-to-period differences in the monitoring data and metrics derived from them. Use of the term significant implies comparison where a statistical test is satisfied at the $p=0.05$ significance level, and NS implies a non-significant test result $(p>0.05)$. Comparison with no mention of significance, using the term numerically implies nominal comparison where one comparator is numerically different from another, without statistical testing. The statistical tests that were performed apply only to absolute differences. Relative differences (changes) are used as a convenient construct to enable discussion of the absolute differences.

\section{Results}

Means of each species-specific value of $\mathrm{P} 4$ atmospheric concentration, Vd, PR, dry, wet, and total deposition, and selected ratios are presented in Table 3 for each site grouping. Corresponding across-site variabilities are given as coefficients of variation (CV). Results are displayed for each grouping of monitoring sites over all seasons and for the east by specific season. P1-to-P4 absolute changes in both native units and relative differences (\%) are displayed in Fig. 4 for each geographical region. To facilitate identification of pat- terns of P1-to-P4 changes, significant $(p \leq 0.05)$ decreases are colored blue, and increases are colored pink. Six sets of period-to-period differences are considered (e.g., P1-to-P2, P1-to-P3); however, they are not tabulated due to space limitations. Although not shown comprehensively, P3-to-P4 differences are discussed in a few instances to contrast with P1to-P4 differences. Graphical illustrations of period-to-period comparisons of concentration, deposition, and related metrics as well as relative changes by site and region have been created for each of the entries shown in Table 3. Example illustrations for selected airborne concentration (i.e., S, OxN, $\mathrm{N}$ and CASTNET PM) and deposition (i.e., total S, total OxN, total $\mathrm{N}$ and wet $\mathrm{H}^{+}$) are shown for all sites in Figs. 5 and 6.

\subsection{Deposition velocity and precipitation rate}

Deposition velocity and its analog, PR, may be viewed as velocities at which pollutants are delivered (i.e., deposited via dry and wet deposition) to the Earth's surface. Both Vd and PR are strongly influenced by meteorology. One of the reasons for selecting 5-year averaging periods in the current study is to reduce the confounding influences of yearto-year meteorological variability in assessing the impacts of changes in pollutant emissions on resulting ambient atmospheric concentrations, dry deposition, and wet deposition.

The variabilities of $\mathrm{Vd}$ and $\mathrm{PR}$ are fairly low. In the case of $\mathrm{Vd}$, this is indicated by the 2010 CASTNET decision (http://epa.gov/castnet/javaweb/docs/ CASTNET_Factsheet_2013.pdf) to discontinue meteorological monitoring and use site-specific historical average $\mathrm{Vd}$ at all but five monitoring sites (Bowker et al., 2011). In the current study, period-site-season-specific CVs, averaged across all periods, sites, and seasons for $\mathrm{Vd}$ for aerosol, $\mathrm{O}_{3}, \mathrm{SO}_{2}$, and $\mathrm{HNO}_{3}$ are each $<5 \%$, and the corresponding value for $\mathrm{PR}$ is $<9 \%$. For the east and for each of the three geographic regions, entity-specific relative differences were determined for each of the six possible period-to-period comparisons (i.e., $4 \times 6=24$ relative differences per entity), yielding median absolute relative differences $\leq 6 \%$ for $\mathrm{Vd}$ of each of the four species and PR. Similar comparisons at the site-specific level ( $34 \times 6=204$ relative differences per entity) yield median absolute relative differences $\leq 7 \%$ for $\mathrm{Vd}$ of each of the four species and PR.

Since period-to-period changes in $\mathrm{Vd}$ are usually small, the behavior of P1-to-P4 changes in species atmospheric concentration and dry deposition are generally similar, and the relative behavior of the dry deposition of a species is often mirrored by that of its airborne concentration. However, there are instances where opposing changes in Vd are sufficient to cause the resulting change in species deposition to differ appreciably and sometimes significantly from its corresponding atmospheric concentration. For example, wintertime increases in aerosol $\mathrm{Vd}$ yield changes in dry deposition that tend to moderate P1-to-P4 reductions apparent in atmo- 
Table 3. Mean (CV) atmospheric concentration, deposition velocity, precipitation rate, dry, wet, and total deposition, and concentration and deposition metrics at 34 eastern US CASTNET and selected subsets of sites for the 5-year period 2005-2009 (see Table 2 for definitions).

\begin{tabular}{|c|c|c|c|c|c|c|c|c|c|c|c|}
\hline \multirow{4}{*}{$\begin{array}{l}\text { Region } \\
\text { season } \\
n\end{array}$} & \multicolumn{11}{|c|}{ Site grouping } \\
\hline & \multicolumn{4}{|c|}{ E } & \multicolumn{2}{|r|}{$\mathrm{NE}$} & \multirow{2}{*}{$\begin{array}{r}\text { MW } \\
\text { OA }\end{array}$} & \multirow{2}{*}{$\begin{array}{l}\text { SO } \\
\text { OA }\end{array}$} & \multirow{2}{*}{$\begin{array}{l}\text { SR } \\
\text { OA }\end{array}$} & \multirow{2}{*}{$\begin{array}{l}\mathrm{HE} \\
\mathrm{OA}\end{array}$} & \multirow{2}{*}{$\begin{array}{r}\mathrm{BKG} \\
\mathrm{OA}\end{array}$} \\
\hline & $\mathrm{OA}$ & W & $\mathrm{Sp}$ & $\mathrm{Su}$ & $\mathrm{F}$ & $\mathrm{OA}$ & & & & & \\
\hline & 34 & 34 & 34 & 34 & 34 & 10 & 10 & 14 & 15 & 3 & 4 \\
\hline & \multicolumn{11}{|c|}{ 2005-2009 Atmospheric concentration, $\mu \mathrm{g} \mathrm{m}^{-3}$} \\
\hline $\mathrm{SO}_{2}$ & $5.63(51)$ & $8.83(55)$ & $5.00(47)$ & $3.75(54)$ & $4.98(52)$ & $7.22(49)$ & $5.87(41)$ & $4.33(48)$ & $7.61(28)$ & $6.45(27)$ & $1.54(16)$ \\
\hline $\mathrm{SO}_{4}$ & $3.52(22)$ & $2.49(18)$ & $3.33(21)$ & $5.13(28)$ & $3.12(21)$ & $3.32(32)$ & $3.41(23)$ & $3.74(12)$ & $3.94(9)$ & $3.49(15)$ & $2.32(39)$ \\
\hline$S($ as $S$ ) & $4.00(40)$ & $5.25(48)$ & $3.61(37)$ & 3.59 (39) & $3.53(41)$ & $4.72(45)$ & $4.08(35)$ & $3.42(34)$ & $5.12(22)$ & $4.39(23)$ & $1.55(25)$ \\
\hline $\mathrm{HNO}_{3}$ & $1.32(32)$ & $1.26(35)$ & $1.56(33)$ & $1.35(40)$ & $1.12(35)$ & $1.33(42)$ & $1.46(24)$ & $1.22(28)$ & $1.51(20)$ & $1.55(10)$ & $0.68(43)$ \\
\hline $\mathrm{NO}_{3}$ & $1.04(64)$ & $1.85(72)$ & $0.92(68)$ & $0.55(36)$ & $0.85(69)$ & $0.78(50)$ & $1.92(17)$ & $0.60(48)$ & $1.38(52)$ & $0.56(25)$ & $0.73(83)$ \\
\hline $\mathrm{OxN}$ (as N) & $0.53(40)$ & $0.70(41)$ & $0.55(41)$ & $0.42(35)$ & 0.44 (44) & $0.47(43)$ & $0.76(15)$ & $0.41(30)$ & $0.65(31)$ & $0.47(1)$ & $0.32(49)$ \\
\hline $\mathrm{NH}_{4}$ & $1.35(26)$ & $1.20(39)$ & $1.20(26)$ & $1.80(28)$ & $1.19(25)$ & $1.23(36)$ & $1.56(17)$ & $1.28(22)$ & $1.58(13)$ & $1.22(6)$ & 0.78 (37) \\
\hline $\mathrm{N}($ as $\mathrm{N})$ & $1.58(29)$ & $1.63(40)$ & $1.49(30)$ & $1.82(26)$ & $1.36(30)$ & $1.43(37)$ & $1.97(16)$ & $1.40(23)$ & $1.88(19)$ & $1.42(4)$ & $0.92(40)$ \\
\hline \multirow[t]{2}{*}{$\mathrm{O}_{3}(\mathrm{ppb})$} & 30.8 (14) & $24.6(16)$ & $38.4(11)$ & $34.5(21)$ & $25.6(18)$ & $30.3(10)$ & $30.6(6)$ & $31.4(20)$ & $30.4(11)$ & $39.4(10)$ & 28.0 (14) \\
\hline & \multicolumn{11}{|c|}{$\mathrm{Vd}, \mathrm{cm} \mathrm{s}^{-1}$} \\
\hline $\mathrm{HNO}_{3}$ & $1.16(21)$ & $0.96(27)$ & $1.31(19)$ & $1.35(19)$ & $1.04(24)$ & $1.13(28)$ & $1.23(11)$ & $1.13(21)$ & $1.19(21)$ & $1.29(7)$ & 1.14 (24) \\
\hline $\mathrm{SO}_{2}$ & $0.31(29)$ & $0.31(40)$ & $0.32(30)$ & $0.33(22)$ & $0.29(32)$ & $0.30(30)$ & $0.36(21)$ & $0.28(32)$ & $0.36(22)$ & $0.30(9)$ & $0.25(27)$ \\
\hline $\mathrm{O}_{3}$ & 0.17 (17) & 0.09 (18) & 0.17 (24) & $0.26(20)$ & 0.15 (18) & $0.16(18)$ & $0.15(15)$ & 0.17 (17) & 0.17 (17) & $0.19(9)$ & 0.16 (19) \\
\hline Aerosol & $0.10(21)$ & $0.08(28)$ & 0.13 (19) & $0.12(22)$ & 0.09 (24) & $0.10(28)$ & $0.11(12)$ & $0.10(22)$ & $0.10(22)$ & $0.11(15)$ & $0.10(18)$ \\
\hline $\mathrm{PR}$ (cm/time) & 119 (19) & $25.7(27)$ & $30.3(24)$ & $32.7(20)$ & $29.6(24)$ & $119(12)$ & $100(20)$ & $132(15)$ & $115(14)$ & $126(17)$ & $124(29)$ \\
\hline
\end{tabular}

\begin{tabular}{|c|c|c|c|c|c|c|c|c|c|c|c|}
\hline \multirow[b]{2}{*}{ Dry $\mathrm{SO}_{2}$ (as $\mathrm{S}$ ) } & \multicolumn{11}{|c|}{ Deposition, $\mathrm{kg}$ (ha yr) $^{-1}$} \\
\hline & $3.13(62)$ & $4.84(70)$ & $2.83(61)$ & $2.20(58)$ & $2.58(62)$ & $3.91(60)$ & $3.68(51)$ & $2.18(61)$ & $4.61(35)$ & $2.96(14)$ & $0.67(12)$ \\
\hline Dry $\mathrm{SO}_{4}$ (as S) & $0.40(33)$ & $0.21(35)$ & $0.46(30)$ & $0.63(37)$ & $0.29(34)$ & $0.36(45)$ & $0.40(31)$ & $0.42(27)$ & $0.44(25)$ & $0.43(23)$ & $0.23(28)$ \\
\hline Dry S (as S) & $3.53(58)$ & $5.06(68)$ & $3.29(55)$ & $2.83(51)$ & $2.87(58)$ & $4.27(58)$ & $4.09(48)$ & $2.60(55)$ & $5.05(33)$ & $3.40(14)$ & $0.91(13)$ \\
\hline Wet S (as S) & $5.22(28)$ & $3.59(35)$ & $5.65(29)$ & $7.53(38)$ & $3.97(26)$ & $5.96(31)$ & $4.97(30)$ & $4.86(20)$ & $6.25(18)$ & $5.13(11)$ & $3.16(31)$ \\
\hline Total S (as S) & $8.75(36)$ & $8.65(47)$ & $8.93(35)$ & $10.4(38)$ & $6.84(35)$ & $10.2(38)$ & $9.06(37)$ & $7.47(25)$ & $11.3(19)$ & $8.53(5)$ & $4.06(27)$ \\
\hline Dry $\mathrm{HNO}_{3}$ (as $\mathrm{N}$ ) & $1.28(41)$ & $0.89(45)$ & $1.65(41)$ & $1.60(45)$ & $0.98(45)$ & $1.24(54)$ & $1.48(35)$ & $1.16(36)$ & $1.48(34)$ & $1.46(18)$ & $0.62(41)$ \\
\hline Dry $\mathrm{NO}_{3}$ (as $\mathrm{N}$ ) & $0.07(66)$ & $0.11(73)$ & $0.09(68)$ & $0.05(48)$ & $0.05(75)$ & $0.06(67)$ & $0.13(21)$ & $0.04(62)$ & $0.10(56)$ & $0.04(20)$ & $0.05(86)$ \\
\hline Dry OxN (as N) & $1.35(41)$ & $1.00(42)$ & $1.74(42)$ & $1.65(44)$ & $1.03(45)$ & $1.30(54)$ & $1.61(33)$ & $1.21(36)$ & $1.58(34)$ & $1.50(18)$ & $0.66(36)$ \\
\hline Wet $\mathrm{OxN}$ (as N) & $2.35(19)$ & $2.06(28)$ & $2.76(22)$ & $2.86(22)$ & $1.73(23)$ & $2.56(24)$ & $2.45(15)$ & $2.14(11)$ & $2.67(12)$ & 2.17 (17) & $1.78(26)$ \\
\hline Total OxN (as N) & $3.71(22)$ & $3.06(25)$ & $4.50(25)$ & $4.51(25)$ & $2.76(24)$ & $3.86(29)$ & $4.06(19)$ & $3.35(13)$ & $4.25(14)$ & $3.67(5)$ & $2.45(28)$ \\
\hline Dry $\mathrm{NH}_{4}$ (as $\mathrm{N}$ ) & $0.35(38)$ & $0.24(49)$ & $0.38(35)$ & $0.52(38)$ & $0.26(39)$ & $0.31(51)$ & $0.41(26)$ & $0.34(37)$ & $0.41(30)$ & $0.35(19)$ & $0.18(32)$ \\
\hline Wet $\mathrm{NH}_{4}$ (as $\mathrm{N}$ ) & $2.30(27)$ & $1.29(33)$ & $3.14(35)$ & $3.10(31)$ & $1.65(34)$ & $2.23(29)$ & $2.87(21)$ & $1.94(14)$ & $2.64(22)$ & $2.33(25)$ & $1.79(54)$ \\
\hline Total $\mathrm{NH}_{4}$ (as N) & $2.65(26)$ & $1.53(32)$ & $3.53(33)$ & $3.62(28)$ & $1.91(31)$ & $2.54(29)$ & $3.28(19)$ & $2.28(15)$ & $3.05(21)$ & $2.68(20)$ & $1.97(52)$ \\
\hline Dry N (as N) & $1.70(40)$ & $1.24(40)$ & $2.12(40)$ & $2.17(41)$ & $1.29(43)$ & $1.61(53)$ & $2.02(31)$ & $1.54(35)$ & $1.98(33)$ & $1.85(18)$ & $0.85(32)$ \\
\hline Wet N (as N) & $4.66(21)$ & $3.36(26)$ & $5.90(27)$ & $5.96(24)$ & $3.38(26)$ & $4.79(25)$ & $5.32(17)$ & $4.08(11)$ & $5.32(14)$ & $4.50(21)$ & $3.58(37)$ \\
\hline Total N (as N) & $6.36(22)$ & $4.59(24)$ & $8.02(26)$ & $8.13(25)$ & $4.67(24)$ & $6.41(28)$ & $7.34(16)$ & $5.63(13)$ & $7.30(15)$ & $6.35(11)$ & $4.42(34)$ \\
\hline Wet $\mathrm{H}^{+}$ & $0.26(37)$ & $0.23(34)$ & $0.23(38)$ & $0.37(49)$ & $0.21(34)$ & $0.34(31)$ & $0.20(41)$ & $0.25(25)$ & $0.31(33)$ & $0.26(8)$ & $0.15(50)$ \\
\hline \multirow[t]{2}{*}{ Dry $\mathrm{O}_{3}$} & $39.5(27)$ & $16.3(28)$ & $48.3(29)$ & $66.1(33)$ & $28.5(31)$ & $38.3(28)$ & $36.6(18)$ & $42.3(30)$ & $40.4(25)$ & $56.4(10)$ & $32.3(18)$ \\
\hline & \multicolumn{11}{|c|}{ Concentration and deposition metrics } \\
\hline $\mathrm{RSO}_{2}$ & $0.67(16)$ & $0.81(11)$ & $0.66(16)$ & $0.49(23)$ & $0.66(17)$ & $0.73(12)$ & $0.71(7)$ & $0.60(18)$ & $0.73(7)$ & $0.73(5)$ & $0.51(17)$ \\
\hline $\mathrm{RFSO}_{2}$ & $0.85(9)$ & $0.93(5)$ & $0.82(12)$ & $0.74(14)$ & $0.86(9)$ & $0.89(6)$ & $0.88(7)$ & $0.80(11)$ & $0.91(3)$ & $0.87(3)$ & $0.75(7)$ \\
\hline$(\mathrm{D} / \mathrm{T}) \mathrm{S}$ & $0.37(33)$ & $0.53(33)$ & $0.35(32)$ & $0.27(36)$ & $0.39(34)$ & $0.38(37)$ & $0.43(20)$ & $0.33(38)$ & $0.44(20)$ & $0.40(14)$ & $0.23(19)$ \\
\hline NI & $0.79(10)$ & $0.70(13)$ & $0.75(12)$ & $0.85(10)$ & $0.80(9)$ & $0.80(8)$ & $0.78(6)$ & $0.78(13)$ & $0.80(7)$ & $0.81(5)$ & $0.67(22)$ \\
\hline $\mathrm{RHNO}_{3}$ & $0.59(22)$ & $0.47(43)$ & 0.65 (19) & $0.69(12)$ & $0.60(23)$ & $0.62(12)$ & $0.42(18)$ & $0.68(12)$ & $0.55(23)$ & $0.73(9)$ & $0.51(33)$ \\
\hline $\mathrm{RNO}_{3}$ & $0.41(32)$ & $0.53(37)$ & $0.35(36)$ & $0.31(28)$ & $0.40(35)$ & $0.38(21)$ & $0.58(13)$ & $0.32(24)$ & $0.45(29)$ & $0.27(26)$ & $0.49(34)$ \\
\hline $\mathrm{RNHNO}_{3}$ & $0.19(18)$ & $0.20(44)$ & $0.23(17)$ & $0.16(27)$ & $0.18(22)$ & $0.20(18)$ & $0.16(15)$ & $0.19(17)$ & $0.18(15)$ & $0.24(6)$ & $0.17(26)$ \\
\hline $\mathrm{RNNH}_{4}$ & $0.67(8)$ & $0.58(6)$ & $0.64(9)$ & $0.76(8)$ & $0.69(10)$ & $0.68(6)$ & $0.61(2)$ & $0.71(6)$ & $0.66(8)$ & $0.67(2)$ & $0.66(6)$ \\
\hline $\mathrm{RFHNO}_{3}$ & $0.95(3)$ & $0.89(11)$ & $0.95(3)$ & $0.97(2)$ & $0.95(3)$ & $0.96(2)$ & $0.91(4)$ & $0.96(1)$ & $0.94(3)$ & $0.97(1)$ & $0.92(7)$ \\
\hline $\mathrm{RNFHNO}_{3}$ & $0.75(6)$ & $0.72(18)$ & $0.77(7)$ & $0.73(8)$ & $0.75(7)$ & $0.76(5)$ & $0.72(7)$ & $0.75(5)$ & $0.75(4)$ & $0.79(1)$ & $0.72(14)$ \\
\hline $\mathrm{RNFNH}_{4}$ & $0.21(15)$ & $0.19(35)$ & $0.19(21)$ & $0.25(22)$ & $0.21(22)$ & $0.20(17)$ & $0.21(15)$ & $0.22(15)$ & $0.21(10)$ & $0.19(2)$ & $0.22(25)$ \\
\hline (D/T) OxN & $0.35(28)$ & $0.32(31)$ & $0.37(27)$ & $0.35(30)$ & $0.36(32)$ & $0.32(38)$ & $0.39(18)$ & $0.35(27)$ & $0.36(25)$ & $0.41(19)$ & $0.27(12)$ \\
\hline$(\mathrm{D} / \mathrm{T}) \mathrm{NH}_{4}$ & $0.13(32)$ & $0.16(39)$ & $0.11(34)$ & $0.15(39)$ & $0.14(38)$ & $0.12(39)$ & $0.13(29)$ & $0.15(30)$ & $0.13(28)$ & $0.14(31)$ & $0.10(18)$ \\
\hline$(\mathrm{D} / \mathrm{T}) \mathrm{N}$ & $0.26(29)$ & $0.27(32)$ & $0.26(30)$ & $0.26(31)$ & $0.27(34)$ & $0.24(38)$ & $0.27(25)$ & $0.27(27)$ & $0.27(26)$ & $0.30(25)$ & 0.19 (17) \\
\hline CASTNET PM & $5.91(24)$ & $5.55(36)$ & $5.45(24)$ & $7.49(26)$ & $5.16(25)$ & $5.33(34)$ & 6.89 (17) & $5.62(16)$ & $6.90(13)$ & $5.28(9)$ & $3.83(32)$ \\
\hline
\end{tabular}




\begin{tabular}{|c|c|c|c|c|c|c|c|c|c|c|c|c|c|c|c|}
\hline Region & eason & C & D & C & $\mathrm{D}$ & $\mathrm{C}$ & D & $\mathrm{D}$ & $\mathrm{D}$ & $\mathrm{C}$ & D & $\mathrm{C}$ & $\mathrm{D}$ & D & D \\
\hline & & $\mathrm{SO} 2$ & Dry SO & SO4 & Dry SO${ }_{4}$ & $S$ & Dry S & Wet S & Total S & 03 & $\mathrm{O}_{3}$ & $\mathrm{NH} 4$ & Dry $\mathrm{NH}_{4}$ & Wet $\mathrm{NH}_{4}$ & Total $\mathrm{NH}_{4}$ \\
\hline $\mathrm{E}$ & $\mathrm{OA}$ & $-5.28(-48)$ & $-3.29(-51)$ & $-1.73(-33)$ & $-0.17(-29)$ & $-3.22(-45)$ & $-3.45(-49)$ & $-2.45(-32)$ & $-5.90(-40)$ & $-1.13(-4)$ & $-1.59(-4)$ & $-0.48(-26)$ & $-0.10(-22)$ & $-0.22(-9)$ & $-0.32(-11)$ \\
\hline$E$ & W & $-8.03(-48)$ & $4.38(-48)$ & $-0.78(-24)$ & $-0.03(-13)$ & $-4.28(-45)$ & $-4.41(-47)$ & $-0.76(-17)$ & $-5.17(-37)$ & 2.04 (9) & 2.65 (19) & $-0.20(-14)$ & $0.00(0)$ & $0.05(4)$ & $0.05(3)$ \\
\hline$E$ & Sp & $-4.45(-47)$ & $-3.24(-53)$ & $-1.50(-31)$ & $-0.16(-26)$ & $-2.73(-43)$ & $-3.40(-51)$ & $-2.79(-33)$ & $-6.19(-41)$ & $-0.16(0)$ & $0.74(2)$ & $-0.48(-29)$ & $-0.11(-23)$ & $-0.29(-8)$ & $-0.40(-10)$ \\
\hline E & Su & $-3.47(-48)$ & $-2.31(-51)$ & $-3.42(-40)$ & $-0.38(-37)$ & $-2.88(-45)$ & $-2.69(-49)$ & $-4.19(-36)$ & $-6.88(-40)$ & $-4.85(-12)$ & $-9.10(-12)$ & $-0.85(-32)$ & $-0.22(-30)$ & $-0.27(-8)$ & $-0.49(-12)$ \\
\hline E & $\mathrm{F}$ & $-5.15(-51)$ & $-2.99(-54)$ & $-1.24(-28)$ & $-0.12(-29)$ & $-2.99(-46)$ & $-3.11(-52)$ & $-2.17(-35)$ & $-5.28(-44)$ & $-1.66(-6)$ & $-3.56(-11)$ & $-0.41(-26)$ & $-0.09(-26)$ & $-0.25(-13)$ & $-0.34(-15)$ \\
\hline MW & $O A$ & $-6.00(-51)$ & $-3.67(-50)$ & $-1.68(-33)$ & $-0.14(-25)$ & $-3.56(-47)$ & $-3.81(-48)$ & $-2.76(-36)$ & $-6.57(-42)$ & $-0.16(-1)$ & $0.34(1)$ & $-0.63(-29)$ & $-0.11(-21)$ & $-0.39(-12)$ & $-0.50(-13)$ \\
\hline MW & w & $-8.55(-50)$ & $-4.90(-47)$ & $-0.90(-26)$ & $-0.02(-7)$ & $-4.58(-47)$ & $-4.92(-46)$ & $0.10(3)$ & $-4.82(-34)$ & $1.47(7)$ & $2.89(25)$ & $-0.24(-12)$ & 0.03 (9) & 0.19 & (13) \\
\hline MW & $\mathrm{Sp}$ & $-4.85(-47)$ & $-3.67(-52)$ & $-1.53(-32)$ & $-0.11(-20)$ & $-2.94(-44)$ & $-3.79(-50)$ & $-3.01(-34)$ & $-6.80(-41)$ & $0.93(3)$ & 4.27 (12) & $-0.68(-32)$ & $-0.11(-20)$ & $-0.45(-10)$ & $-0.56(-11)$ \\
\hline MW & Su & $-4.68(-54)$ & $-2.99(-55)$ & $-3.38(-42)$ & $-0.33(-35)$ & $-3.47(-49)$ & $-3.32(-52)$ & $-4.68(-43)$ & $-7.99(-46)$ & $-2.44(-6)$ & $-3.11(-5)$ & $-1.14(-40)$ & $-0.26(-33)$ & $-0.38(-9)$ & $-0.64(-13)$ \\
\hline MW & $\mathrm{F}$ & $-5.87(-52)$ & $-3.74(-53)$ & $-0.94(-23)$ & $-0.06(-15)$ & $-3.25(-46)$ & $-3.80(-51)$ & $-2.69(-39)$ & $-6.49(-45)$ & $-0.91(-4)$ & $-1.14(-4)$ & $-0.46(-25)$ & $-0.06(-17)$ & $-0.59(-22)$ & $-0.65(-21)$ \\
\hline $\mathrm{NE}$ & $\mathrm{OA}$ & $-6.61(-48)$ & $-4.18(-52)$ & $-1.74(-34)$ & $-0.18(-33)$ & $-3.89(-45)$ & $-4.36(-51)$ & $-2.55(-30)$ & $-6.91(-40)$ & $-1.12(-4)$ & $-3.43(-8)$ & $-0.45(-27)$ & $-0.11(-26)$ & $-0.18(-7)$ & $-0.29(-10)$ \\
\hline NE & w & $-10.3(-45)$ & $-6.25(-49)$ & $-0.801-$ & $-0.05(-19)$ & $-5.43(-43)$ & $-6.30(-48)$ & $-0.76(-19)$ & $-7.05(-41)$ & 2.93 & $2.82(22)$ & $-0.18(-14)$ & $-0.02(-7)$ & -0.06 & -0.0 \\
\hline NE & Sp & $-5.41(-48)$ & $(-57)$ & $-1.50(-34)$ & $-0.17(-29)$ & $-3.21(-45)$ & $-4.24(-55)$ & -3.3 & $-7.57(-45)$ & $0.27(1)$ & $0.49(1)$ & $-0.43(-29)$ & $-0.11(-25)$ & -0.42 & -15) \\
\hline NE & Su & $-4.30(-48)$ & -3.01 & $-3.45(-41)$ & $-0.43(-42)$ & $-3.31(-45)$ & $-3.44(-51)$ & $(-30)$ & $-7.71(-37)$ & $-6.65(-17)$ & $-11.9(-15)$ & $-0.82(-32)$ & $-0.25(-34)$ & -0.28 & $(-13)$ \\
\hline NE & $\mathrm{F}$ & $-6.39(-51)$ & $-4.10(-58)$ & $-1.29(-32)$ & $-0.13(-37)$ & $-3.63(-48)$ & $-4.23(-56)$ & $(-37)$ & $-6.79(-47)$ & $-0.87(-3)$ & -3.11 & $-0.39(-28)$ & $-0.10(-33)$ & -0.08 & -0.1 \\
\hline so & $\mathrm{OA}$ & $-3.81(-47)$ & -2.37 & -1.75 & $-0.17(-29)$ & $-2.49(-$ & -2.55( & -2.1 & -4.71( & $-1.82(-5)$ & -1.65 & $-0.40(-24)$ & $-0.09(-21)$ & -0.1 & \\
\hline so & w & $-6.04(-48)$ & $-3.28(-52)$ & $-0.69(-22)$ & $-0.03(-12)$ & $-3.25(-45)$ & $-3.31(-50)$ & $-1.44(-28)$ & $-4.75(-40)$ & $1.81(7)$ & $2.36(15)$ & $-0.18(-16)$ & $-0.01(-4)$ & $-0.15(-10)$ & $-0.16(-10)$ \\
\hline so & Sp & $-3.49(-46)$ & $-2.61(-54)$ & $-1.48(-29)$ & $-0.19(-28)$ & $-2.24(-41)$ & $-2.80(-51)$ & $-2.12(-28)$ & $-4.92(-37)$ & $-1.24(-3)$ & $-1.60(-3)$ & $-0.38(-25)$ & $-0.11(-23)$ & -0.06 & -0.1 \\
\hline so & Su & $-2.02(-41)$ & $(-43)$ & $-3.42(-38)$ & -0.38 & $-2.16(-39)$ & $-1.67(-41)$ & -3.3 & $-5.03(-36)$ & $-5.28(-14)$ & $-11.5(-15)$ & $-0.66(-26)$ & $-0.16(-24)$ & -0.07 & -0.2 \\
\hline \multirow[t]{2}{*}{ so } & $\mathrm{F}$ & $-3.74(-50)$ & $-2.17(-56)$ & $-1.43(-30)$ & $-0.16(-34)$ & $-2.35(-44)$ & $-2.33(-53)$ & $-1.66(-32)$ & $-3.99(-42)$ & $-2.75(-9)$ & $-5.62(-15)$ & $-0.39(-26)$ & $-0.10(-29)$ & $-0.18(-12)$ & $-0.28(-16)$ \\
\hline & & HNO3 & Dry $\mathrm{HNO}_{3}$ & NO3 & Dry $\mathrm{NO}_{3}$ & OxN & & & Total OxN & & & $\mathrm{N}$ & Dry N & & \\
\hline$E$ & $\mathrm{OA}$ & $-0.67(-34)$ & $-0.69(-35)$ & $-0.09(-8)$ & $0.00(-1)$ & $-0.17(-24)$ & $-0.69(-34)$ & $-0.97(-29)$ & $-1.66(-31)$ & $1.61(1)$ & $-0.24(-47)$ & $-0.55(-26)$ & $-0.79(-32)$ & $-1.19(-20)$ & $-1.98(-24)$ \\
\hline$E$ & w & $-0.41(-24)$ & $-0.26(-23)$ & $0.20(12)$ & $0.03(31)$ & $-0.04(-6)$ & $-0.24(-19)$ & $-0.32(-13)$ & $-0.56(-15)$ & $1.37(6)$ & -0.11( & $-0.20(-11)$ & $-0.24(-16)$ & $-0.27(-7)$ & $-0.51(-10)$ \\
\hline$E$ & Sp & $-0.66(-30)$ & $-0.72(-30)$ & $-0.21(-19)$ & $-0.01(-8)$ & $-0.19(-26)$ & $-0.73(-29)$ & $-1.22(-31)$ & $-1.94(-30)$ & $-0.07(0)$ & $-0.28(-55)$ & $-0.57(-28)$ & $-0.84(-28)$ & $-1.50(-20)$ & $-2.34(-23)$ \\
\hline $\mathrm{E}$ & Su & $-0.97(-42)$ & $-1.15(-42)$ & $-0.17(-24)$ & $-0.01(-22)$ & $-0.26(-38)$ & $-1.23(-43)$ & -1.4 & $-2.66(-37)$ & $0.98(3)$ & $-0.36(-50)$ & $-0.91(-33)$ & $-1.46(-40)$ & -1.70 & $(-28)$ \\
\hline$E$ & $\mathrm{~F}$ & $-0.64(-37)$ & $-0.60(-38)$ & $-0.19(-18)$ & $-0.01(-14)$ & $-0.19(-30)$ & $-0.60(-37)$ & -0.8 & $-1.45(-34)$ & $0.61(2)$ & $-0.20(-49)$ & $-0.51(-27)$ & $-0.69(-35)$ & -1.09 & $(-28)$ \\
\hline MW & $\mathrm{OA}$ & $-0.63(-30)$ & $-0.58(-28)$ & $-0.29(-13)$ & $0.00(-2)$ & $-0.21(-21)$ & $-0.59(-27)$ & $-0.96(-28)$ & $-1.55(-28)$ & 1.75 & $-0.24(-56)$ & $-0.70(-26)$ & $-0.70(-26)$ & $-1.35(-20)$ & $-2.04(-22)$ \\
\hline MW & w & $-0.44(-30)$ & $-0.22(-22)$ & $0.27(8)$ & $0.05(32)$ & $-0.04(-3)$ & $-0.17(-15)$ & $-0.08(-4)$ & $-0.25(-8)$ & $5.20(33)$ & $-0.06(-21)$ & $-0.23(-9)$ & $-0.14(-10)$ & $0.11(3)$ & $-0.04(-1)$ \\
\hline MW & Sp & $-0.51(-22)$ & $-0.47(-20)$ & $-0.64(-27)$ & $-0.03(-14)$ & $-0.26(-24)$ & $-0.49(-19)$ & $-1.12(-27)$ & $-1.62(-24)$ & $1.61(6)$ & $-0.29(-62)$ & $-0.78(-29)$ & $-0.60(-19)$ & $-1.57(-18)$ & $-2.18(-19)$ \\
\hline MW & Su & $-1.08(-38)$ & $-1.10(-34)$ & $-0.34(-30)$ & $-0.02(-21)$ & $-0.32(-36)$ & $-1.12(-34)$ & $-1.40(-32)$ & $-2.52(-33)$ & $-1.43(-5)$ & $-0.39(-65)$ & $-1.20(-39)$ & $-1.38(-34)$ & $-1.78(-$ & $-3.16(-25)$ \\
\hline MW & $\mathrm{F}$ & $-0.46(-27)$ & $-0.41(-26)$ & $-0.42(-21)$ & $-0.01(-13)$ & $-0.20(-23)$ & $-0.43(-26)$ & $-1.05(-36)$ & $-1.48(-32)$ & $-2.30(-9)$ & $-0.22(-54)$ & $-0.55(-24)$ & $-0.49(-24)$ & -1.63 & $-2.13(-28)$ \\
\hline $\mathrm{NE}$ & $\mathrm{OA}$ & $-0.84(-39)$ & $-0.88(-41)$ & $-0.01(-1)$ & $0.00(2)$ & $-0.19(-29)$ & $-0.88(-40)$ & $-1.31(-34)$ & $-2.19(-36)$ & $9.81(9)$ & $-0.26(-44)$ & $-0.54(-27)$ & $-0.98(-38)$ & $-1.49(-24)$ & $-2.48(-28)$ \\
\hline NE & w & $-0.48(-25)$ & $-0.43(-31)$ & 0.22 (19) & $0.02(29)$ & $-0.06(-8)$ & $-0.41(-29)$ & $-0.58(-20)$ & $-0.99(-23)$ & $2.95(13)$ & $-0.11(-30)$ & $-0.19(-12)$ & $-0.43(-26)$ & $-0.64(-16)$ & $-1.07(-18)$ \\
\hline NE & Sp & $-0.90(-38)$ & $-1.00(-39)$ & $-0.04(-6)$ & $0.00(3)$ & $-0.21(-30)$ & $-0.99(-38)$ & $-1.86(-41)$ & $-2.85(-40)$ & $-0.03(0)$ & $-0.33(-55)$ & $-0.54(-29)$ & $-1.11(-36)$ & $-2.28(-30)$ & $-3.39(-31)$ \\
\hline NE & Su & $-1.20(-48)$ & $-1.54(-50)$ & $-0.12(-21)$ & $-0.01(-23)$ & $-0.29(-43)$ & $-1.55(-50)$ & $-1.78(-36)$ & $-3.33(-42)$ & 3.21 (11) & $-0.39(-42)$ & $-0.93(-34)$ & $-1.81(-47)$ & $-2.06(-25)$ & $-3.87(-31)$ \\
\hline $\mathrm{NE}$ & $\mathrm{F}$ & $-0.80(-42)$ & $-0.81(-47)$ & $-0.09(-13)$ & $-0.01(-14)$ & $-0.20(-34)$ & $-0.81(-46)$ & $6(-35)$ & $-1.87(-39)$ & $2.87(10)$ & $-0.25(-50)$ & $-0.50(-30)$ & $-0.91(-44)$ & $-1.14(-23)$ & $-2.05(-30)$ \\
\hline so & $\mathrm{OA}$ & $-0.58(-32)$ & $-0.63(-35)$ & $-0.01(-2)$ & $0.00(-1)$ & $-0.13(-24)$ & $-0.63(-34)$ & $-0.70(-25)$ & $-1.33(-29)$ & $-3.12(-2)$ & $-0.22(-46)$ & $-0.44(-24)$ & $-0.72(-32)$ & $-0.85(-17)$ & $-1.57(-22)$ \\
\hline so & w & $-0.34(-20)$ & $-0.24(-21)$ & $0.15(18)$ & $0.02(32)$ & $-0.04(-7)$ & $-0.22(-19)$ & $-0.43(-19)$ & $-0.65(-19)$ & $-3.56(-11)$ & $-0.17(-43)$ & $-0.18(-13)$ & $-0.23(-16)$ & $-0.57(-16)$ & $-0.81(-16)$ \\
\hline so & Sp & $-0.59(-29)$ & $-0.78(-34)$ & $-0.03(-6)$ & $0.00(-3)$ & $-0.14(-24)$ & $-0.78(-33)$ & $-0.71(-21)$ & $-1.49(-26)$ & $-0.24(-1)$ & $-0.23(-50)$ & $-0.43(-25)$ & $-0.89(-31)$ & $-0.77(-12)$ & $-1.66(-18)$ \\
\hline so & Su & $-0.74(-40)$ & $-0.88(-41)$ & $-0.09(-16)$ & $-0.01(-20)$ & $-0.18(-35)$ & $-1.00(-43)$ & $-1.12(-30)$ & $-2.11(-35)$ & 0.78 & $-0.31(-46)$ & $-0.69(-28)$ & $-1.20(-39)$ & $-1.19(-19)$ & $-2.41(-26)$ \\
\hline so & $\mathrm{F}$ & $-0.66(-39)$ & $-0.69(-44)$ & $-0.09(-16)$ & $-0.01(-16)$ & $-0.17(-33)$ & $-0.70(-43)$ & $-0.56(-28)$ & $-1.25(-35)$ & $0.76(2)$ & $-0.16(-45)$ & $-0.47(-28)$ & $-0.80(-40)$ & $-0.73(-21)$ & $-1.53(-28)$ \\
\hline
\end{tabular}

Figure 4. P1-to-P4 changes expressed in Table 3 units and as relative differences (\%); see Table 2 for definitions of column headings.

spheric concentration (e.g., dry $\mathrm{SO}_{4}$; Fig. 4) and exaggerate increases (e.g., dry $\mathrm{NO}_{3}$ ).

In the east and in each geographic region, seasonal P1-toP4 changes in mean PR are not significant ( $p \leq 0.05$; Fig. 4), and they are usually small $(\leq 10 \%)$, except in winter. These wintertime changes contribute to a small, but significant, $1 \%$ increase in PR in the east, $9 \%$ increase in the northeast, and $2 \%$ decrease in the south. Reduced significance of wintertime changes in wet deposition (e.g., wet $\mathrm{S}$, wet OxN, wet $\mathrm{H}^{+}$, and wet N; Fig. 4) appears to be associated with these numerical wintertime P1-to-P4 changes in PR.

\subsection{Oxidized sulfur species}

As is illustrated in Fig. 4, significant P1-to-P4 reductions occur in atmospheric $\mathrm{SO}_{2}$ concentration and dry deposition, atmospheric aerosol $\mathrm{SO}_{4}$ concentration, atmospheric $\mathrm{S}$ concentration and dry deposition, and total $\mathrm{S}$ in each region in each season and over all seasons. For atmospheric $\mathrm{SO}_{2}$ concentration and dry deposition, the numerically largest significant seasonal absolute reductions usually occur in winter when $\mathrm{SO}_{2}$ concentration is at its seasonal maximum (largest in northeast); the smallest occur in summer when $\mathrm{SO}_{2}$ concentration is at its seasonal minimum (smallest in south). Both $\mathrm{SO}_{2}$ concentration and dry deposition show similar P1to-P4 relative reductions of approximately $50 \%$ across all seasons and regions.

As noted earlier, P1-to-P4 changes in atmospheric aerosol $\mathrm{SO}_{4}$ concentrations show significant reductions in each region both in each season and over all seasons. Seasonally, the numerically largest absolute and relative reductions tend to occur in summer when aerosol $\mathrm{SO}_{4}$ concentration is at its seasonal maximum, with regionally uniform summertime absolute reductions of $\approx 3.4 \mu \mathrm{g} \mathrm{SO}_{4} \mathrm{~m}^{-3}$ and relative reductions ranging regionally between 38 and $42 \%$. The regionality of aerosol $\mathrm{SO}_{4}$ concentration, a secondary pollutant, is also illustrated in Table 3 by contrasting its across-site variability $(\mathrm{CV})$ with that of its precursor, $\mathrm{SO}_{2}$ (e.g., $22 \%$ vs. $51 \%)$.

Dry deposition of aerosol $\mathrm{SO}_{4}$ also shows significant $\mathrm{P} 1$ to-P4 reductions regionally. Overall relative reductions in 
a

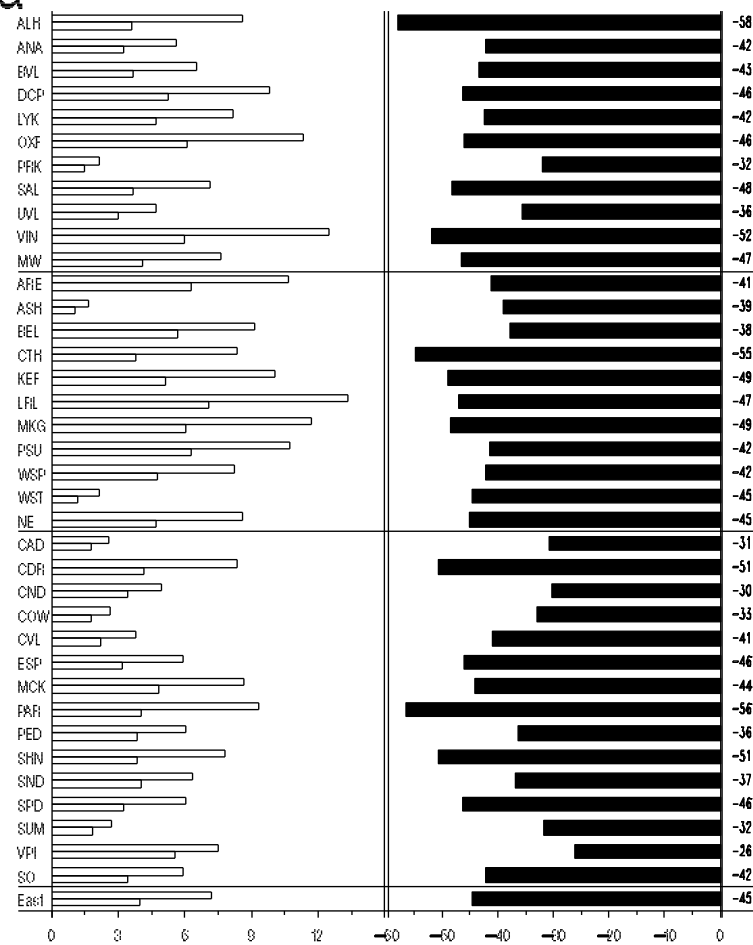

c

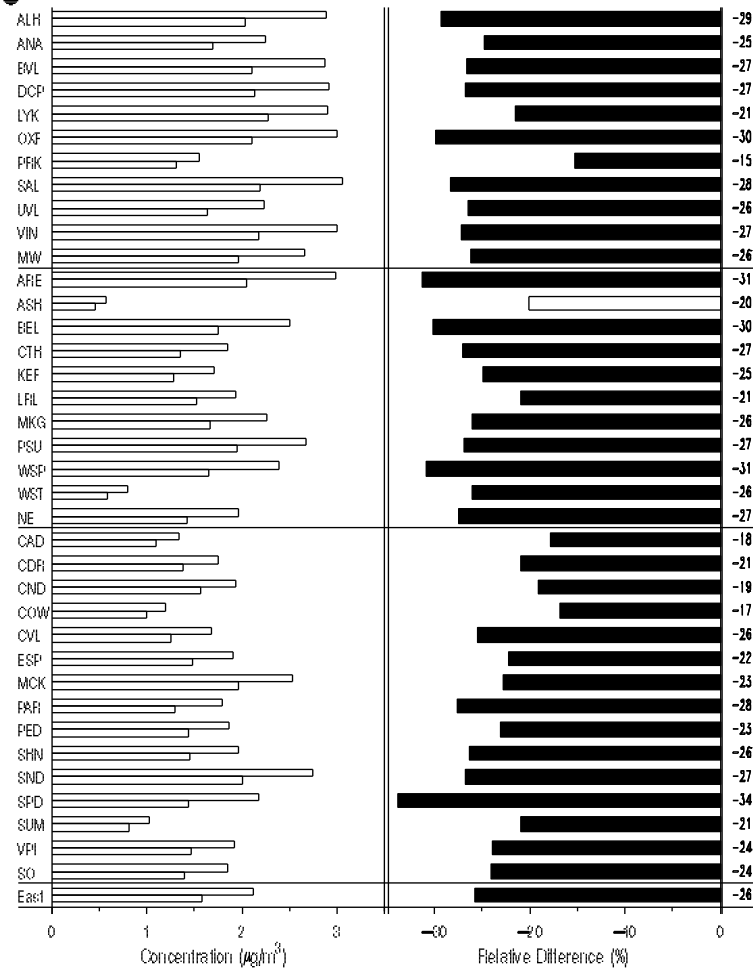

b

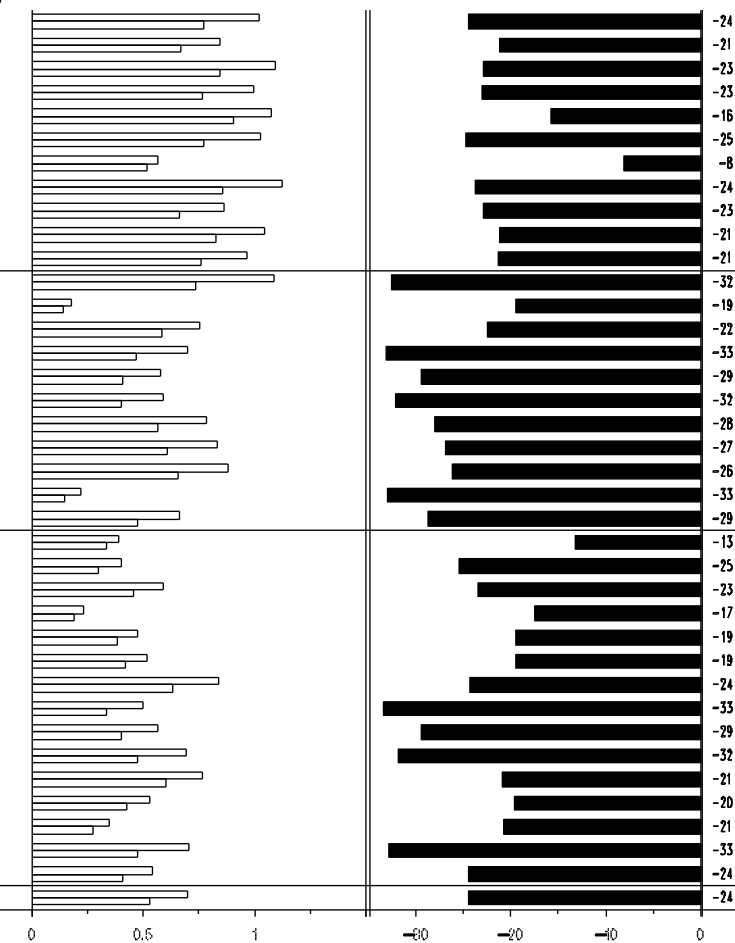

d

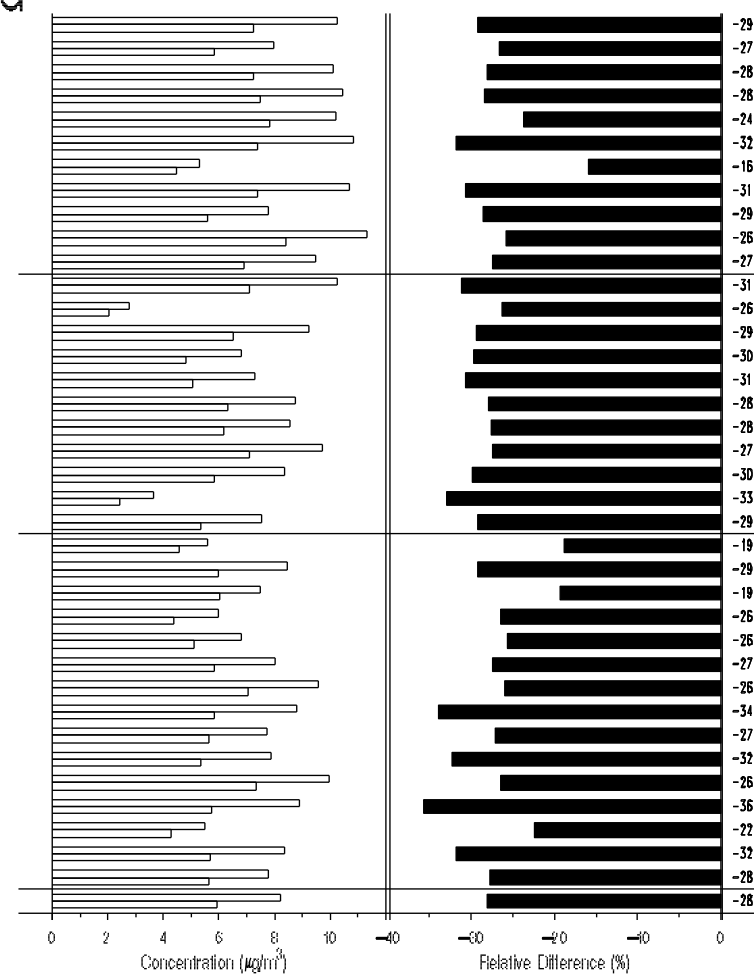

Figure 5. Site- and region-specific concentration $\left(\mu \mathrm{g} \mathrm{m}^{-3}\right.$, left - upper bar, $\mathrm{P} 1$; and lower bar, $\left.\mathrm{P} 4\right)$ and $\mathrm{P} 1$-to-P4 relative changes (\%, right and bottom) for (a) S: monitored oxidized sulfur $\left(\mu \mathrm{g} \mathrm{S} \mathrm{m}{ }^{-3}\right)$; (b) OxN: monitored oxidized nitrogen $\left(\mu \mathrm{g} \mathrm{N} \mathrm{m}{ }^{-3}\right.$ ); (c) $\mathrm{N}$ : monitored oxidized plus reduced nitrogen $\left(\mu \mathrm{g} \mathrm{N}^{-3}\right)$; and (d) CASTNET PM: sum of aerosol $\mathrm{SO}_{4}, \mathrm{NO}_{3}$, and $\mathrm{NH}_{4}\left(\mu \mathrm{g} \mathrm{m}^{-3}\right)$. Significance level of period-to-period relative changes indicated by bar fill: black, $p \leq 0.05$; and open, $p>0.05$ (NS). Sites are identified by region in the same order as shown in Table 1 . 
a

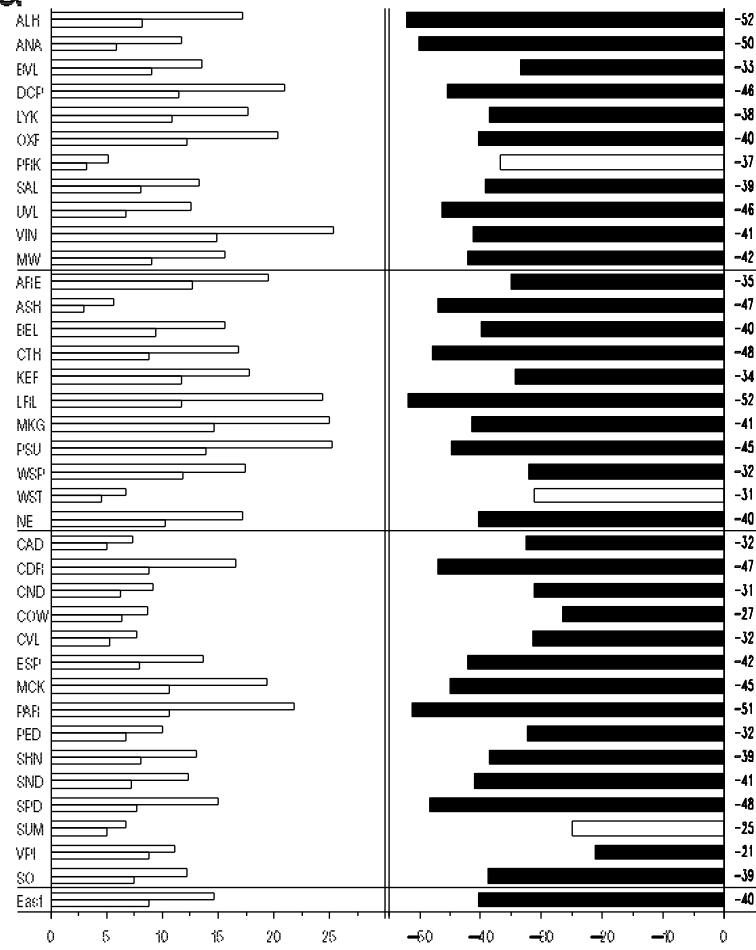

C

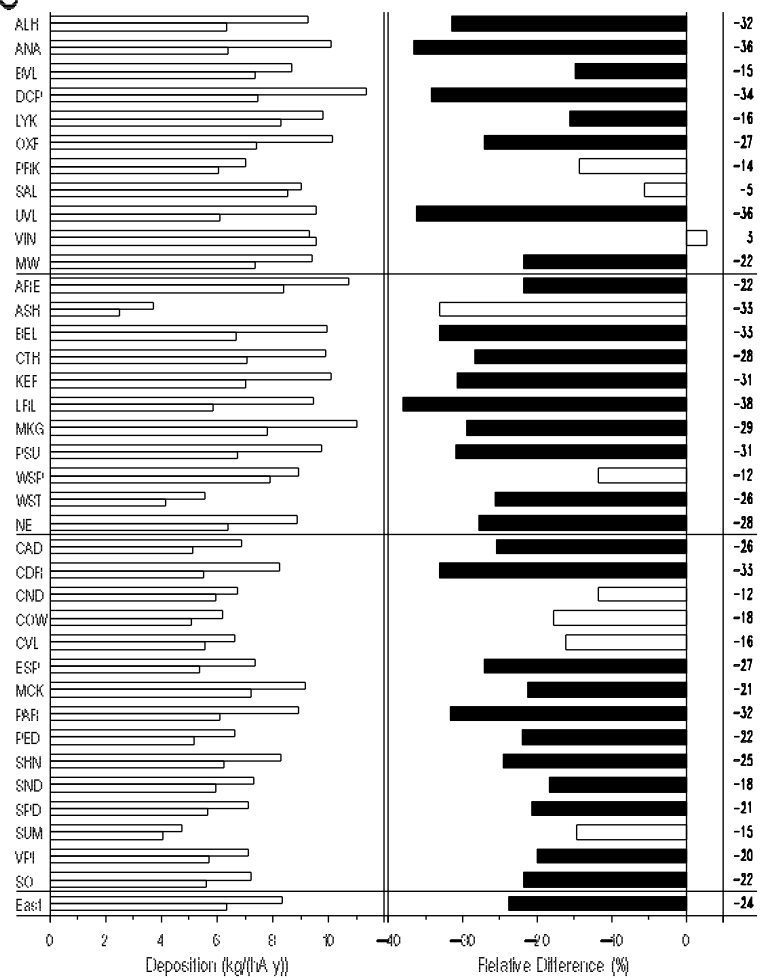

$b$

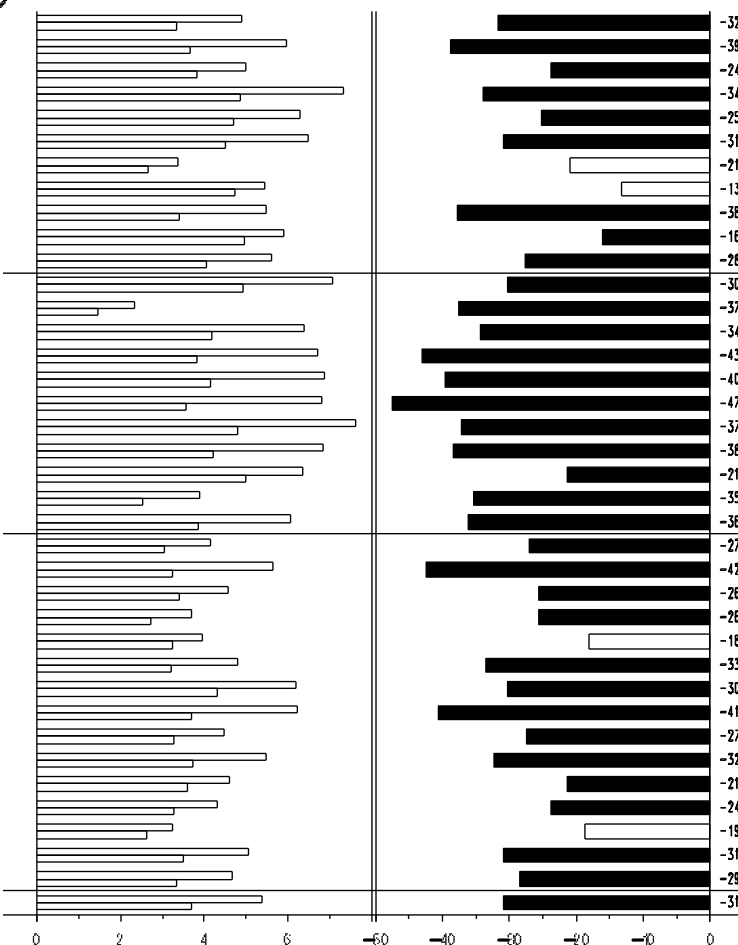

d

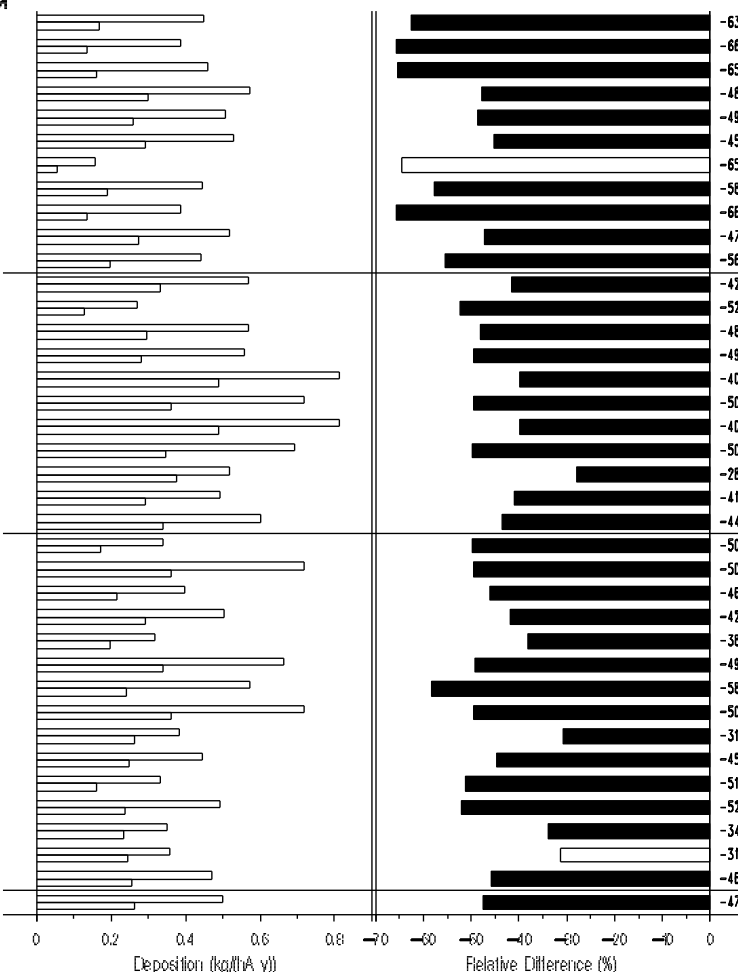

Figure 6. Site- and region-specific total deposition (kg (ha yr) $)^{-1}$, left - upper bar, P1; and lower bar, P4) and P1-to-P4 relative changes (\%, right and bottom) for (a) Total S: total dry plus wet oxidized sulfur deposition; (b) Total OxN: sum of dry plus wet deposition of monitored oxidized nitrogen; (c) Total N: sum of total dry plus wet deposition of monitored oxidized plus reduced nitrogen; and (d) Wet $\mathrm{H}^{+}$: wet deposition of $\mathrm{H}^{+}$. Significance level of period-to-period relative changes indicated by bar fill: black, $p \leq 0.05$; and open, $p>0.05$ (NS). Sites are identified by region in the same order as shown in Table 1. 
dry $\mathrm{SO}_{4}$ are numerically less than the corresponding values for concentration of aerosol $\mathrm{SO}_{4}$ (i.e., for the east, $29 \%$ vs. $33 \%)$. This pattern is likely due in part to significant period-to-period increases in aerosol Vd, noted earlier. Seasonally, the numerically largest absolute and relative reductions in dry $\mathrm{SO}_{4}$ tend to occur in summer when aerosol $\mathrm{SO}_{4}$ concentration is at its seasonal maximum, with an average relative reduction of $37 \%$ in summer in the east. Dry $\mathrm{SO}_{4}$ shows significant reductions in most seasons, except winter, when its concentration is at a seasonal minimum.

Since atmospheric $\mathrm{S}$ concentration is the sum of atmospheric $\mathrm{SO}_{2}$ and aerosol $\mathrm{SO}_{4}$ concentrations (expressed as mass of sulfur) and $\approx 67 \%$ of atmospheric $\mathrm{S}$ is present as $\mathrm{SO}_{2}\left(\mathrm{RSO}_{2}\right.$; Table 3), the behavior of atmospheric $\mathrm{S}$ concentration is dominated by that of $\mathrm{SO}_{2}$. Additionally, since $\approx 85 \%$ of the dry $\mathrm{S}$ is usually contributed by $\mathrm{SO}_{2}\left(\mathrm{RFSO}_{2}\right.$; Table 3), the behavior of dry $\mathrm{S}$ is likewise dominated by that of dry $\mathrm{SO}_{2}$. As a result, P1-to-P4 changes of both atmospheric $\mathrm{S}$ concentration and dry deposition are similar to those for atmospheric $\mathrm{SO}_{2}$ concentration and dry deposition, and they are similar to each other (Fig. 4). Atmospheric S concentration and dry $\mathrm{S}$ are reduced significantly in each region in each season and over all seasons. Seasonally, the numerically largest significant absolute reductions usually occur in winter when atmospheric $\mathrm{SO}_{2}$ concentration is at its seasonal maximum, and the largest of these occurs in the northeast. Regionally, P1-to-P4 relative reductions are similar for atmospheric S concentration (42 to $47 \%$ ) and dry deposition (48 to $51 \%$ ).

In contrast to atmospheric $\mathrm{SO}_{2}$ concentration (with its summertime minimum), wet $\mathrm{S}$ has a seasonal summer maximum. This coincides with scavenging of $\mathrm{SO}_{4}$ by precipitation when both atmospheric aerosol $\mathrm{SO}_{4}$ concentration and $\mathrm{PR}$ are generally at their seasonal maxima. The numerically largest significant absolute and relative P1-to-P4 reductions in wet $\mathrm{S}$ also generally occur in summer. Changes in wet $\mathrm{S}$ are numerically smaller and less significant in winter due to seasonal minima of wet S and PR and to wintertime P1-to-P4 increases in PR. In general, relative reductions in wet $\mathrm{S}$ are similar to those of aerosol $\mathrm{SO}_{4}$ concentration (e.g., 33 and $32 \%$ in the east).

Except during winter, wet $\mathrm{S}$ generally constitutes more than $50 \%$ of the total S ((D/T) S; Table 3) and exerts a corresponding influence on total S. As noted earlier, P1-to-P4 total $\mathrm{S}$ is reduced significantly in each region in each season and over all seasons (Fig. 4). Since seasonal behaviors of dry $\mathrm{S}$ and wet $\mathrm{S}$ are different but complementary, the seasonal behavior of their sum, total $\mathrm{S}$, shows less seasonal variability than its constituents. Nevertheless, the numerically largest significant P1-to-P4 absolute reductions for total S occur in summer when wet $\mathrm{S}$ is at its seasonal maximum. Relative P1to-P4 reductions are similar across regions, and average $40 \%$ in the east.

\subsection{Oxidized nitrogen species}

Sampling artifacts described in Sect. 2 suggest that sampling artifacts may provide biases in opposing directions for concentrations of $\mathrm{HNO}_{3}$ and aerosol $\mathrm{NO}_{3}$, but these impacts would be minimized during non-summer sampling at nonmarine sites. Regional and seasonal changes in atmospheric $\mathrm{HNO}_{3}$ concentration have more consistent, substantial, and significant reductions for P1-to-P4 (Fig. 4) than for P1-to-P2 or P1-to-P3 (not shown). With recent summertime reductions in $\mathrm{NO}_{\mathrm{x}}$ emissions, the seasonal timing of peak $\mathrm{HNO}_{3}$ concentration has shifted from summer in P1 and P2 to spring in $\mathrm{P} 3$ and $\mathrm{P} 4$. In addition, $\mathrm{P} 1, \mathrm{P} 2$, and $\mathrm{P} 3$ summertime peaks of dry deposition of both $\mathrm{HNO}_{3}$ and $\mathrm{OxN}$ have shifted to spring in $\mathrm{P} 4$. The overall $\mathrm{P} 1$-to-P4 relative reductions in atmospheric $\mathrm{HNO}_{3}$ concentration in the east are $34 \%$ vs. $0 \%$ (NS) for P1-to-P2 and $13 \%$ for P1-to-P3. Regionally, the numerically largest significant $\mathrm{P} 1$-to-P4 reductions occur in the northeast (39\%), with smaller reductions in the south $(32 \%)$ and midwest $(30 \%)$. Numerically, maximum absolute and relative seasonal reductions consistently occur in summer, with the largest of these in the northeast. Summertime P1-to$\mathrm{P} 4$ relative reductions in atmospheric $\mathrm{HNO}_{3}$ concentration are $48 \%$ in northeast, $40 \%$ in south, $38 \%$ in midwest, and $42 \%$ in east. Wintertime P1-to-P4 absolute reductions in atmospheric $\mathrm{HNO}_{3}$ concentration are smaller, 40 to $46 \%$ of those in summer.

Dry $\mathrm{HNO}_{3}$ exhibits P1-to-P4 reductions that are of similar magnitude and consistency with those noted above for atmospheric $\mathrm{HNO}_{3}$ concentration. The only exception is winter in the midwest, where $\mathrm{P} 4 \mathrm{Vd}$ was $9 \%$ higher than in $\mathrm{P} 1$. Regionally, significant reductions occur in the northeast $(41 \%)$, south $(35 \%)$, and midwest (28\%), averaging $35 \%$ in the east. Seasonal behavior of dry $\mathrm{HNO}_{3}$ is similar to that of atmospheric $\mathrm{HNO}_{3}$ concentration, with numerically maximum absolute and relative seasonal reductions generally occurring in summer. Summertime P1-to-P4 relative reductions in dry $\mathrm{HNO}_{3}$ are $50 \%$ in northeast, $41 \%$ in south, $34 \%$ in midwest, and $42 \%$ in east. Wintertime P1-to-P4 absolute reductions in dry $\mathrm{HNO}_{3}$ are smaller, 20 to $28 \%$ of those in summer.

In contrast to P1-to-P3 comparisons, where atmospheric aerosol $\mathrm{NO}_{3}$ concentration and dry deposition generally display significant increases (SS07b), P1-to-P4 comparisons in Fig. 4 show more reductions. Significant P1-to-P4 absolute and relative increases in atmospheric aerosol $\mathrm{NO}_{3}$ concentration and dry $\mathrm{NO}_{3}$ occur only in winter in the east (e.g., $0.20 \mu \mathrm{g} \mathrm{NO}_{3} \mathrm{~m}^{-3}$ and $12 \%$, and $0.03 \mathrm{kgN}$ (ha yr) ${ }^{-1}$ and $31 \%$ ) when atmospheric aerosol $\mathrm{NO}_{3}$ concentration is at its seasonal maximum. The increased magnitude of winter relative increases in dry $\mathrm{NO}_{3}$ over those of airborne concentration are likely due to previously noted significant period-to-period increases in aerosols Vd. Wintertime regional absolute P1-to-P4 increases in concentration and dry deposition are numerically strongest in the midwest (e.g., $0.27 \mu \mathrm{g} \mathrm{NO}_{3} \mathrm{~m}^{-3}$ and $0.05 \mathrm{kgN}$ (ha yr) $)^{-1}$ ). The wintertime 
a

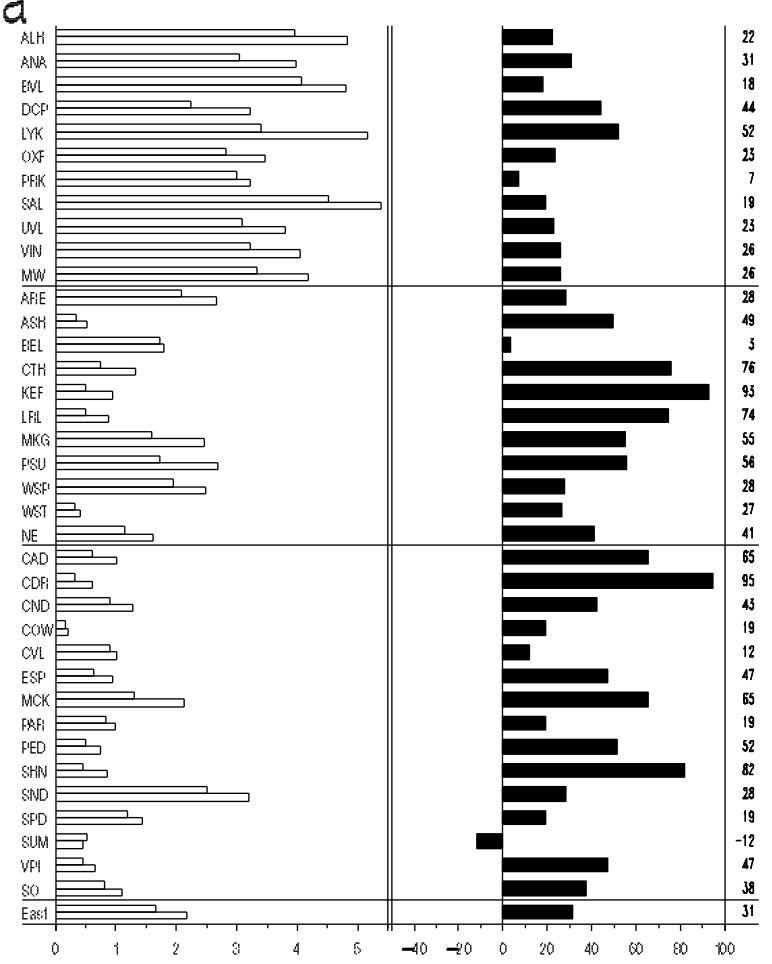

C

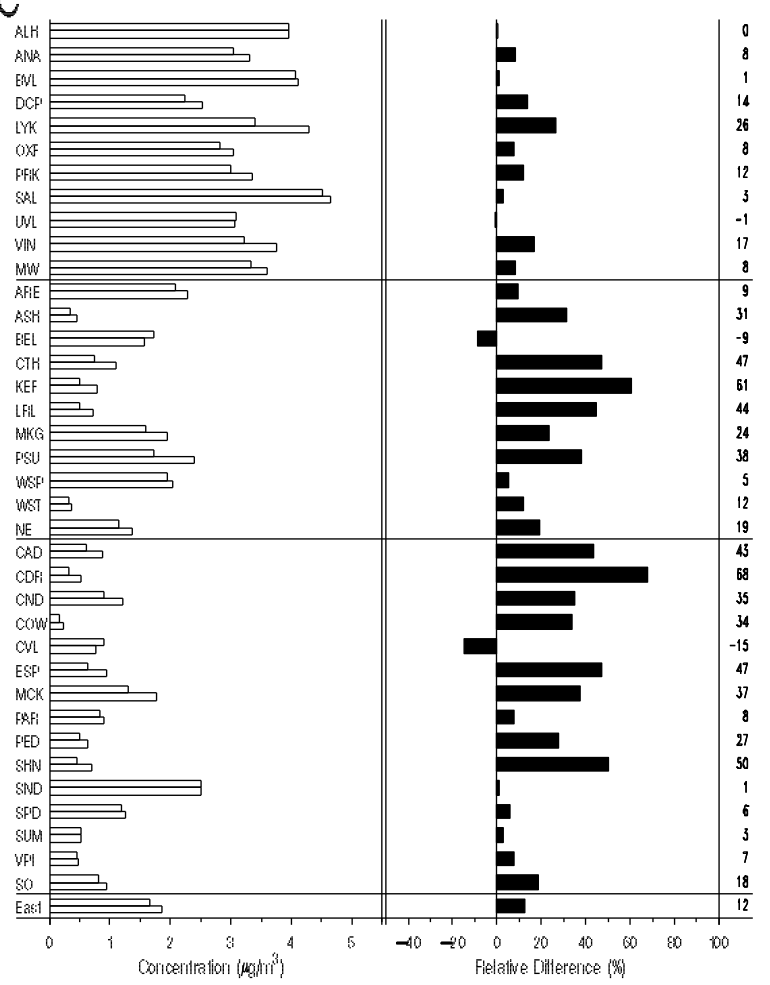

b

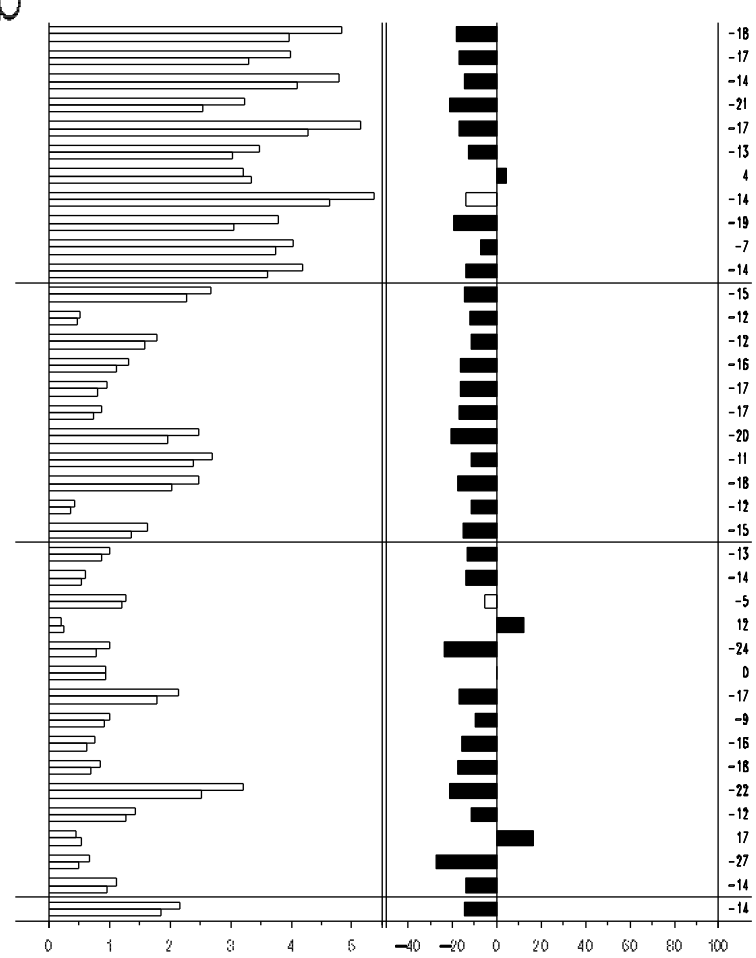

d

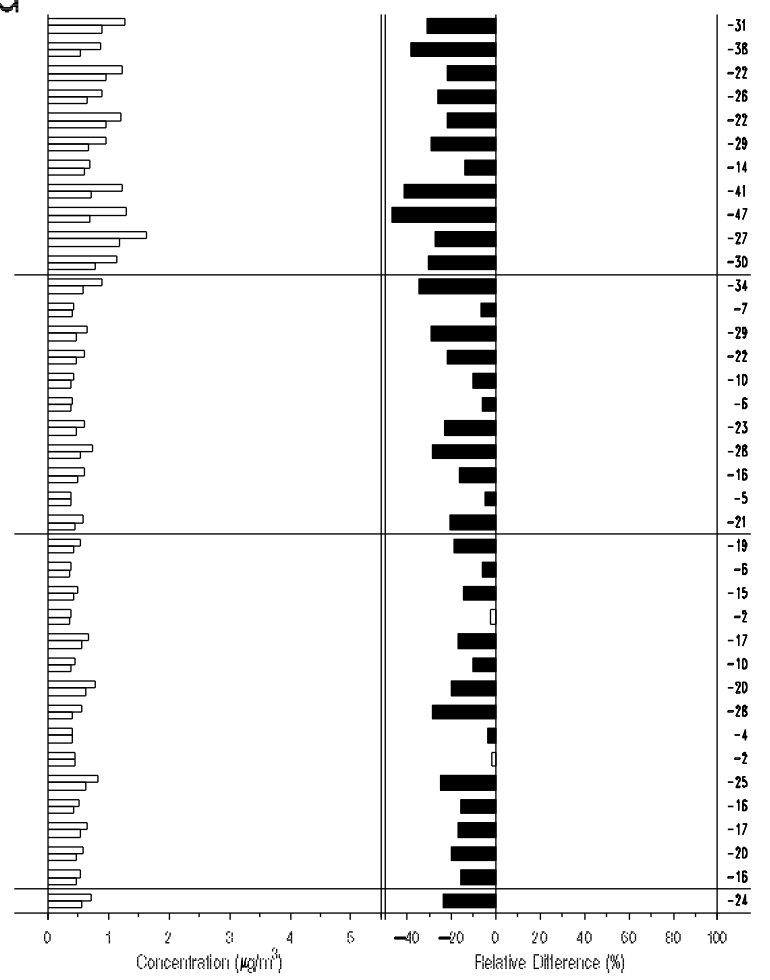

Figure 7. Site- and region-specific seasonal aerosol $\mathrm{NO}_{3}$ concentration $\left(\mu \mathrm{g} \mathrm{m}^{-3}\right.$, left - upper bar: early period; and lower bar: later period) and period-to-period relative changes (\%, right and bottom) for (a) P1-to-P3, W; (b) P3-to-P4, W; (c) P1-to-P4, W; and (d) P1-to-P4, Su. Significance level of period-to-period relative changes indicated by bar fill: black, $p \leq 0.05$; and open, $p>0.05$ (NS). Sites are identified by region in the same order as shown in Table 1. 
P1-to-P4 increases in aerosol $\mathrm{NO}_{3}$ concentration are considerably smaller than the corresponding P1-to-P3 increases (Fig. 7). Significant P1-to-P4 reductions in absolute and relative atmospheric aerosol $\mathrm{NO}_{3}$ concentration occur in many of the remaining seasons, especially summer. Significant P3-to$\mathrm{P} 4$ reductions in relative atmospheric aerosol $\mathrm{NO}_{3}$ concentration occur in all seasons in the east, consistent with recent aggressive reductions of $\mathrm{NO}_{\mathrm{x}}$ emissions.

Atmospheric $\mathrm{OxN}$ concentration is the sum of atmospheric $\mathrm{HNO}_{3}$ and aerosol $\mathrm{NO}_{3}$ concentrations (expressed as mass of nitrogen), and almost $60 \%$ of the OxN is generally present as $\mathrm{HNO}_{3}\left(\mathrm{RHNO}_{3}\right.$; Table 3). As a result, the behavior of atmospheric OxN concentration reflects the influence of both atmospheric $\mathrm{HNO}_{3}$ and aerosol $\mathrm{NO}_{3}$ concentrations. Also, since well over $90 \%$ of the dry OxN is generally contributed by $\mathrm{HNO}_{3}\left(\mathrm{RFHNO}_{3}\right.$; Table 3$)$, the behavior of dry $\mathrm{OxN}$ is strongly influenced by that of dry $\mathrm{HNO}_{3}$. Seasonally in the east, the numerically largest P1-to-P4 absolute and relative reductions in atmospheric OxN concentration and dry deposition (e.g., $0.26 \mu \mathrm{g} \mathrm{N} \mathrm{m}^{-3}$ and $38 \%$, and $1.23 \mathrm{~kg} \mathrm{~N}$ (ha yr) ${ }^{-1}$ and $43 \%$; Fig. 4) occur in summer when atmospheric $\mathrm{HNO}_{3}$ is the dominant OxN species. Summertime relative reductions are numerically more pronounced in the northeast (43 and $50 \%$, respectively) than the south ( 35 and $43 \%$ ) or midwest (36 and $34 \%)$. In contrast, in winter, when atmospheric aerosol $\mathrm{NO}_{3}$ concentration is at its seasonal maximum and significant P1-to-P4 increases of both atmospheric aerosol $\mathrm{NO}_{3}$ concentration and dry deposition occur, reductions in OxN concentration and dry deposition also occur, but they are smaller in magnitude and in some cases not significant. Over all seasons, regional P1-to-P4 relative reductions in atmospheric OxN concentration and dry deposition are numerically larger in the northeast (29 and $40 \%$ ) than the south (24 and $34 \%$ ) or the midwest ( 21 and $27 \%$ ), and average 24 and $34 \%$, respectively, in the east.

Wet OxN and PR in the east are at their seasonal maxima in summer (followed closely by spring). Numerically, the largest significant absolute reductions in wet $\mathrm{OxN}$ occur in summer (or spring). Reduced significance of some wintertime $\mathrm{P} 1$-to-P4 reductions in wet $\mathrm{OxN}$ is associated with numerical increases in wintertime PR. Significant regional $\mathrm{P} 1$-to-P4 reductions in wet $\mathrm{OxN}$ are numerically larger in the northeast $(34 \%)$ than midwest $(28 \%)$ or south $(25 \%)$, and average $29 \%$ in the east.

Considering the dry deposition of a comprehensive suite of $\mathrm{NO}_{\mathrm{y}}$ species, field measurements suggest that 40 to $85 \%$ of total $\mathrm{NO}_{\mathrm{y}}$ deposition at eight rural Canadian sites occurred as wet deposition (Zhang et al., 2009), whereas a modeling study suggests that for the continental US this figure is closer to $31 \%$ for $\mathrm{NO}_{\mathrm{y}}$ and $44 \%$ for OxN (Zhang et al., 2012). In the current study, over $60 \%$ of the total OxN generally is contributed by wet OxN ((D/T) OxN; Table 3); however, this estimate may be biased because it neglects previously noted uncertainties in $\mathrm{Vd}$ for $\mathrm{HNO}_{3}$ and contributions to dry deposition by non-monitored $\mathrm{NO}_{\mathrm{y}}$ species. Nevertheless, wet
OxN appears to exert a moderate-to-strong influence on the behavior of total OxN. Both dry OxN and wet OxN display strong seasonal P1-to-P4 reductions in summer that become weaker in winter (Fig. 4). Seasonally, the numerically largest absolute and relative reductions in total $\mathrm{OxN}$ occur in summer and range from $42 \%$ in the northeast, to $35 \%$ in the south, to $33 \%$ in the midwest, and average $37 \%$ in the east. Regional P1-to-P4 reductions in total OxN are $36 \%$ in the northeast, $29 \%$ in the south, and $28 \%$ in the midwest, and average $31 \%$ in the east. Thus, over all seasons the numerically largest absolute and relative reductions in atmospheric $\mathrm{HNO}_{3}$ concentration and dry deposition, atmospheric OxN concentration and dry deposition, and total OxN generally occur in the northeast, and seasonally they tend to occur there in summer.

\subsection{Reduced nitrogen species}

Since $\mathrm{NH}_{3}$ is not monitored in CASTNET, aerosol $\mathrm{NH}_{4}$ is the reduced nitrogen species considered in the current study. Numerically, the regional maximum for atmospheric aerosol $\mathrm{NH}_{4}$ concentration occurs near emissions sources in the source region and midwest, and the seasonal maximum in the east occurs in summer. As is illustrated in Fig. 4, between $\mathrm{P} 1$ and $\mathrm{P} 4$, atmospheric aerosol $\mathrm{NH}_{4}$ concentration shows significant regional relative reductions that are similar, ranging from $29 \%$ in the midwest, to $27 \%$ in the northeast, to $24 \%$ in the south, and averaging $26 \%$ in the east. Seasonally, the numerically largest absolute and relative reductions of atmospheric aerosol $\mathrm{NH}_{4}$ concentration occur in summer (when atmospheric aerosol $\mathrm{SO}_{4}$ concentration is at its seasonal maximum and is also experiencing its largest absolute and relative reductions). Summertime P1-to-P4 relative reductions in atmospheric aerosol $\mathrm{NH}_{4}$ concentration range from $40 \%$ in the midwest, to $32 \%$ in the northeast, to $26 \%$ in the south, and average $32 \%$ in the east. In contrast, reductions are smaller in winter (e.g., $14 \%$ in the east) when atmospheric aerosol $\mathrm{NH}_{4}$ concentration is small and atmospheric aerosol $\mathrm{SO}_{4}$ concentration is also at its seasonal minimum.

Although generally showing significant reductions, dry deposition of atmospheric aerosol $\mathrm{NH}_{4}$ shows numerically weaker P1-to-P4 changes than its atmospheric concentration. The overall $\mathrm{P} 1$-to-P4 relative reduction for dry $\mathrm{NH}_{4}$ in the east $(22 \%)$ is numerically smaller than the corresponding value for its atmospheric concentration. This pattern of weaker reductions is likely due to the significant P1-to-P4 increases in aerosol Vd, noted earlier. Regional P1-to-P4 relative reductions in dry $\mathrm{NH}_{4}$ are $26 \%$ in the northeast and $21 \%$ in the midwest and south. Seasonally, in winter, when the atmospheric aerosol $\mathrm{NH}_{4}$ concentration is low, changes in its dry deposition show changes which tend to be small and not significant regionally. Mirroring aerosol $\mathrm{NH}_{4}$ concentration, the numerically largest $\mathrm{P} 1$-to-P4 absolute and relative reductions in dry $\mathrm{NH}_{4}$ also occur in summer. They range 
from $34 \%$ in the northeast, to $33 \%$ in the midwest, to $24 \%$ in the south, and average $30 \%$ in the east.

Numerically, wet $\mathrm{NH}_{4}$ is also high near emissions sources in the midwest and source region, and in the east in the spring and summer seasons when PR is also relatively high. At the regional and seasonal scales, wet $\mathrm{NH}_{4}$ shows P1-to-P4 changes that are usually not significant. The only two reductions that are significant occur overall in the midwest $(12 \%)$ and east $(9 \%)$. Two wintertime increases occur in the midwest and the east, but they are not significant.

Since the magnitude of wet $\mathrm{NH}_{4}$ is over seven times larger than dry deposition ((D/T) $\mathrm{NH}_{4}$; Table 3), the behavior of total $\mathrm{NH}_{4}$ is strongly influenced by the behavior of wet $\mathrm{NH}_{4}$. Similar to wet $\mathrm{NH}_{4}$, total $\mathrm{NH}_{4}$ shows mostly P1-to-P4 reductions that are usually not significant, but with non-significant wintertime increases in the midwest and the east. The only significant reduction is overall in the east $(11 \%)$.

\subsection{Oxidized plus reduced nitrogen species}

The sum of monitored oxidized and reduced nitrogen species is represented as $\mathrm{N}$. The behavior of the resulting $\mathrm{N}$ metrics (i.e., atmospheric $\mathrm{N}$ concentration, dry $\mathrm{N}$, wet $\mathrm{N}$, and total $\mathrm{N}$ ) reflects the behavior of the weighted sum of the individual constituents, described previously.

Aerosol $\mathrm{NH}_{4}$ constitutes $\approx 67 \%, \mathrm{HNO}_{3} \approx 19 \%$, and aerosol $\mathrm{NO}_{3} \approx 14 \%$ of the monitored airborne $\mathrm{N}$ concentration $\left(\mathrm{RNNH}_{4}\right.$ and $\mathrm{RNHNO}_{3}$; Table 3). As a result, aerosol $\mathrm{NH}_{4}$ exerts a moderate-to-strong influence on its behavior. Seasonally, the numerically strongest absolute and relative P1-to-P4 reductions of atmospheric $\mathrm{N}$ concentration occur in summer (midwest, 39\%; northeast, 34\%; south, $28 \%$; and east, $33 \%$ ). This seasonality coincides with the summer peaks of atmospheric $\mathrm{N}$ concentration and $\mathrm{P} 1$-to-P4 reductions in the concentration of aerosol $\mathrm{NH}_{4}, \mathrm{HNO}_{3}$, and $\mathrm{OxN}$. Regionally, atmospheric $\mathrm{N}$ concentration shows relative reductions of $26 \%$ in the midwest, $27 \%$ in the northeast, $24 \%$ in the south, and $26 \%$ in the east.

Due to the large difference in deposition velocities, $\approx 75 \%$ of dry $\mathrm{N}$ is contributed by dry $\mathrm{HNO}_{3}$, but only $\approx 21 \%$ by dry $\mathrm{NH}_{4}$ and $\approx 4 \%$ by dry $\mathrm{NO}_{3}\left(\mathrm{RNFHNO}_{3}\right.$ and $\mathrm{RNFNH}_{4}$; Table 3 ). Seasonally, the numerically strongest absolute and relative $\mathrm{P} 1-$ to- $\mathrm{P} 4$ reductions in dry $\mathrm{N}$ mirror those of aerosol $\mathrm{NH}_{4}, \mathrm{HNO}_{3}$, and $\mathrm{OxN}$ concentration and dry deposition (and $\mathrm{N}$ concentration); they occur in summer (northeast, $47 \%$; south, $39 \%$; midwest, $34 \%$; and east, $40 \%$ ). Regional non-significant wintertime $\mathrm{P} 1-$ to-P4 reductions may result in part from corresponding significant regional increases in dry $\mathrm{NO}_{3}$. Regionally, the numerically largest P1-to-P4 reductions occur in the northeast $(38 \%)$, with smaller reductions in the south $(32 \%)$ and midwest $(26 \%)$, and they average $32 \%$ in the east.

Wet $\mathrm{OxN}$ and wet $\mathrm{NH}_{4}$ contribute similarly to wet $\mathrm{N}$, with maxima in spring or summer (Table 3). The numerically largest significant P1-to-P4 absolute reductions in wet
$\mathrm{N}$ also occur in spring or summer, and peak seasonal relative reductions range between 20 and $30 \%$. Non-significant numerical wintertime $\mathrm{P} 1-$ to-P4 reductions in wet $\mathrm{N}$ occur in the northeast and south, with a non-significant increase in the midwest. This behavior is likely associated with previously noted numerical wintertime P1-to-P4 increases in PR. Regional P1-to-P4 relative reductions in wet $\mathrm{N}$ are $24 \%$ in the northeast, $20 \%$ in the midwest, $17 \%$ in the south, and average $20 \%$ in the east.

Since wet $\mathrm{N}$ contributes $\approx 75 \%$ of total N ((D/T) N; Table 3), the behavior of total $\mathrm{N}$ is strongly influenced by the behavior of wet $\mathrm{N}$. The numerically largest significant $\mathrm{P} 1$-to-P4 absolute reductions in total $\mathrm{N}$ generally occur in summer, with corresponding seasonal relative reductions of $31 \%$ in northeast, $25 \%$ in midwest, and $26 \%$ in south. Nonsignificant $\mathrm{P} 1$-to-P4 reductions in total $\mathrm{N}$ occur only in wintertime and are associated with similar behavior of wet $\mathrm{OxN}$ and wet $\mathrm{N}$ and numerical wintertime P1-to-P4 increases in $\mathrm{PR}$. Regionally, the numerically largest $\mathrm{P} 1$-to-P4 reductions occur in the northeast $(28 \%)$, with smaller reductions in the midwest and south $(22 \%)$. The overall P1-to-P4 relative reduction of total $\mathrm{N}$ in the east is $24 \%$.

\subsection{Wet $\mathrm{H}^{+}$}

Regionally, wet $\mathrm{H}^{+}$deposition is generally heaviest in the northeast, and seasonally in the east it is heaviest during summer (Table 3). Seasonally, the numerically largest absolute reductions occur in summer, with relative reductions of similar magnitude in spring and summer, while generally smaller reductions occur in winter (Fig. 4). The numerically largest seasonal relative reductions range from $65 \%$ in the midwest (summer), to $55 \%$ in the northeast (spring), to $50 \%$ in the south (spring). Regionally, P1-to-P4 regional relative reductions of wet $\mathrm{H}^{+}$are numerically larger near sources in the midwest $(56 \%)$ and smaller in the south $(46 \%)$ and northeast $(44 \%)$. The overall $\mathrm{P} 1-$ to-P4 relative reduction in wet $\mathrm{H}^{+}$in the east is $47 \%$.

\subsection{Atmospheric $\mathrm{O}_{3}$ concentration and dry deposition}

In the current study, hourly atmospheric $\mathrm{O}_{3}$ concentrations, averaged to weekly values, are treated as other weekly CASTNET data (i.e., averaged to period-site-season means); however, this does not represent the relevant time period for determining compliance with the US ambient air quality standard. As is illustrated in Fig. 4, mostly nonsignificant $\mathrm{P} 1$-to-P4 seasonal increases in atmospheric $\mathrm{O}_{3}$ concentration occur in winter in the northeast (13\%, NS), midwest and south ( $7 \%$, NS), and east (9\%, significant). Summertime atmospheric $\mathrm{O}_{3}$ concentrations do show substantial and mostly significant absolute and relative reductions in each region: $6.7 \mathrm{ppb}(17 \%)$ in the northeast, $5.3 \mathrm{ppb}$ (14\%) in the south, $2.4 \mathrm{ppb}(6 \%$, NS) in the midwest, and $4.9 \mathrm{ppb}(12 \%)$ in the east. The numerically largest reduc- 
tion in atmospheric $\mathrm{O}_{3}$ concentration occurs in summer and in the northeast, coinciding with aggressive $\mathrm{O}_{3}$-season $\mathrm{NO}_{\mathrm{x}}$ emissions controls during $\mathrm{P} 3$ and $\mathrm{P} 4$ in this region and upwind in the east. Unpublished examination of seasonal $\mathrm{NO}_{\mathrm{x}}$ emissions in the eastern US for the period between 19901992 and 2006-2008 (roughly corresponding to P1-to-P4) suggests that reductions in summertime emissions are approximately $6 \%$ greater than those for the aggregate of the remaining three seasons over the same time period. Regional reductions of atmospheric $\mathrm{O}_{3}$ concentration over all seasons are small and only significant in the south $(5 \%)$ and east $(4 \%)$.

Moderate P1-to-P4 increases in $\mathrm{Vd}$ for $\mathrm{O}_{3}$ act in opposition to corresponding reductions in atmospheric $\mathrm{O}_{3}$ concentration to yield P1-to-P4 changes in dry deposition of $\mathrm{O}_{3}$ that are sometimes significant. Relatively large, significant seasonal reductions in dry $\mathrm{O}_{3}$, ranging from 5 to $15 \%$, occur regionally in summer; however, corresponding increases ranging between 15 and $25 \%$ occur in winter. Regionally, $\mathrm{P} 1$-to-P4 reductions in dry $\mathrm{O}_{3}$ occur in the northeast $(8 \%)$ and south (4\%), and a non-significant increase occurs in the midwest (1\%, NS). The P1-to-P4 relative reduction in dry $\mathrm{O}_{3}$ in the east is $4 \%$.

\section{Discussion}

\subsection{Changes in atmospheric aerosol $\mathrm{SO}_{4}$ and $\mathrm{SO}_{2}$ concentration}

Both P1-to-P4 absolute and relative changes in atmospheric aerosol $\mathrm{SO}_{4}$ concentration, shown in Fig. 4, are numerically substantially less than the corresponding values for atmospheric $\mathrm{SO}_{2}$ concentration, consistent with the findings of Reid et al. (2001), Holland et al. (2004), and SS07b for earlier time periods. For regional groupings of sites and in the east, the year-round ratios of P1-to-P4 relative reductions in atmospheric aerosol $\mathrm{SO}_{4}$ concentration to atmospheric $\mathrm{SO}_{2}$ concentration are similar $\left(\approx 0.7 \%\right.$ decrease in aerosol $\mathrm{SO}_{4}$ for each $1 \%$ decrease in $\mathrm{SO}_{2}$ ). In the east these ratios range numerically between a wintertime low $\left(\approx 0.5 \% \mathrm{SO}_{4}\right.$ per $1 \%$ $\left.\mathrm{SO}_{2}\right)$ and a high $\left(\approx 0.8 \% \mathrm{SO}_{4}\right.$ per $\left.1 \% \mathrm{SO}_{2}\right)$ in summer when aerosol $\mathrm{SO}_{4}$ levels are at their seasonal maxima and $\mathrm{SO}_{2}$ levels are at their seasonal minima. Year-round ratios of P1-to-P4 absolute reductions of atmospheric aerosol $\mathrm{SO}_{4}$ concentration to those of $\mathrm{SO}_{2}$ concentration (both expressed as mass of sulfur) are similar in regions of higher emissions density, the midwest and northeast $\left(\approx 0.2 \mu \mathrm{g} \mathrm{S} \mathrm{m}{ }^{-3}\right.$ decrease in aerosol $\mathrm{SO}_{4}$ for each $1 \mu \mathrm{g} \mathrm{S} \mathrm{m}{ }^{-3}$ decrease in $\left.\mathrm{SO}_{2}\right)$. This ratio is numerically higher in the south $(\approx 0.3)$, and it may be due in part to concurrent reductions of $\mathrm{SO}_{2}$ emissions in areas of higher emissions density with subsequent transport of reduced amounts of oxidized sulfur, especially aerosol $\mathrm{SO}_{4}$, into the south.
$\mathrm{RSO}_{2}$ (Table 3) is the ratio of atmospheric $\mathrm{SO}_{2}$ concentration to total atmospheric $\mathrm{S}$ concentration, and $1-\mathrm{RSO}_{2}$ is the relative amount of atmospheric $\mathrm{S}$ concentration present as aerosol $\mathrm{SO}_{4}$, representing a rough index of the extent of conversion of $\mathrm{SO}_{2}$ to other oxidized sulfur species (i.e., aerosol $\mathrm{SO}_{4}$ ). Significant $\mathrm{P} 1$-to-P4 $\mathrm{RSO}_{2}$ reductions (not shown) of $8 \%$ in the south, $5 \%$ in the northeast, and $7 \%$ in the midwest and east correspond to increases in the conversion index (e.g., $17 \%$ in the east). This suggests that as the P1-to-P4 atmospheric $\mathrm{SO}_{2}$ concentration in the east has decreased, the relative amount of airborne $\mathrm{S}$ present as $\mathrm{SO}_{2}$ has decreased, and both the relative amount present as aerosol $\mathrm{SO}_{4}$ and the apparent extent of conversion of $\mathrm{SO}_{2}$ to aerosol $\mathrm{SO}_{4}$ have increased.

\subsection{Changes in dry and wet deposition}

As noted in Sect. 3.2, wet $\mathrm{S}$ exceeds dry $\mathrm{S}$ in the east, except in winter. The $\mathrm{P} 4$ ratio of dry $\mathrm{S}$ to total $\mathrm{S}$ in the east is 0.37 ((D/T) S; Table 3), down from 0.44 in P1; further, qualitatively similar behavior is noted for the BKG sites (i.e., P4 ratio 0.23 vs. 0.26 in $\mathrm{P} 1$ ) and $\mathrm{HE}$ sites (i.e., $\mathrm{P} 4$ ratio 0.40 vs. 0.50 in P1). In the east, the ratio of dry $\mathrm{S}$ to wet $\mathrm{S}$ (calculated from $(\mathrm{D} / \mathrm{T}) \mathrm{S})$ has diminished by $24 \%$ from P1 $(0.78)$ to $\mathrm{P} 4(0.60)$. This reduction is strongest $(>30 \%)$ in regions of high emissions density (midwest and northeast) in winter, where $\mathrm{SO}_{2}$ concentrations are at their peak. Year-round, in the east and regionally, both P1-to-P4 absolute and relative reductions in dry $\mathrm{S}$ are numerically larger (by $\approx 20$ to $70 \%$; Fig. 4) than corresponding values for wet S. However, in summer when PR is high and atmospheric aerosol $\mathrm{SO}_{4}$ concentration is at its seasonal maximum, the absolute P1to-P4 reductions are larger (by $\approx 25$ to $100 \%$ ) for wet $\mathrm{S}$ than dry S. In other seasons, the roles are different, and in winter, when atmospheric $\mathrm{SO}_{2}$ concentration is at its seasonal maximum and PR is at its seasonal minimum, P1-to-P4 absolute and relative reductions in dry $\mathrm{S}$ are substantially larger (by over a factor of $\approx 2$ ) than the corresponding reductions for wet $\mathrm{S}$. Thus, strong emissions controls of the primary pollu$\operatorname{tant} \mathrm{SO}_{2}$ have reduced dry $\mathrm{S}$ to a greater extent than wet $\mathrm{S}$, permitting the latter to become an increasing relative contributor to total S. This, along with the increased apparent conversion of $\mathrm{SO}_{2}$ to aerosol $\mathrm{SO}_{4}$, noted above, suggests that wet processes are becoming more prominent than dry processes at removing oxidized sulfur from the current atmosphere in the east.

Whereas deposition estimates of both primary and secondary oxidized sulfur species are available for comparison of their dry and wet P1-to-P4 changes, only changes in deposition of monitored secondary oxidized nitrogen species can be compared in the current study. In the east, dry OxN appears to be numerically smaller than wet OxN (Sect. 3.3), and the $\mathrm{P} 4$ ratio of dry OxN to total $\mathrm{OxN}$ is 0.35 ((D/T) OxN; Table 3), down slightly from 0.37 in P1. Qualitatively similar behavior is noted for the BKG sites (i.e., P4 ratio 0.27 
vs. 0.30 in $\mathrm{P} 1$ ) and $\mathrm{HE}$ sites (i.e., $\mathrm{P} 4$ ratio 0.41 vs. 0.51 in $\mathrm{P} 1)$. Absolute $\mathrm{P} 1$-to-P4 reductions in dry $\mathrm{OxN}$ are generally numerically smaller than corresponding values for wet $\mathrm{OxN}$ (Fig. 4). However, with the recent, more aggressive $\mathrm{NO}_{\mathrm{x}}$ emissions controls, the relative P1-to-P4 reductions of dry OxN now exceed those for wet OxN more frequently than in the earlier P1-to-P3 comparison (SS07b). These findings suggest that wet $\mathrm{OxN}$ is becoming an increasing relative contributor to total OxN.

\subsection{CASTNET PM}

Although $\mathrm{PM}_{2.5}$ is not monitored in CASTNET, many of the major inorganic constituents (i.e., aerosol $\mathrm{SO}_{4}, \mathrm{NO}_{3}$, and $\mathrm{NH}_{4}$ ) are monitored. In contrast to aerosol $\mathrm{SO}_{4}$ and $\mathrm{NH}_{4}$, which occur mainly as fine particles, there is evidence suggesting that aerosol $\mathrm{NO}_{3}$ can be multi-modal in the east and is subject to sampling artifacts in opposing directions with the CASTNET sampler, but they are thought to be minimal during cooler seasons at the non-marine, eastern CASTNET sites (see Sect. 2). In the current study, CASTNET PM is taken to be the sum of atmospheric aerosol $\mathrm{SO}_{4}, \mathrm{NO}_{3}$, and $\mathrm{NH}_{4}$ mass concentrations.

Significant P1-to-P4 (Fig. 5) and P3-to-P4 reductions occur in CASTNET PM concentration in each region in each season and over all seasons. This is in contrast to P1-to-P3 behavior where non-significant wintertime changes occurred (SS07b), with both numerical and significant increases in the midwest (Fig. 8). Year-round absolute and relative reductions in the east are $2.3 \mu \mathrm{g} \mathrm{m}^{-3}$ and $28 \%$ (P1-to-P4) and $1.1 \mu \mathrm{g} \mathrm{m}^{-3}$ and $16 \%$ (P3-to-P4). Seasonally, they peak in summer when aerosol $\mathrm{SO}_{4}$ and $\mathrm{NH}_{4}$ concentrations are at their maxima and are also experiencing their largest reductions. Regionally, the largest absolute and relative reductions in CASTNET PM occur in the midwest in summer $\left(4.9 \mathrm{\mu g} \mathrm{m}^{-3}\right.$ and $41 \%-\mathrm{P} 1$-to-P4 and $1.9 \mu \mathrm{g} \mathrm{m}^{-3}$ and $21 \%$ - P3-to-P4).

For the chemically complex $\mathrm{NH}_{3}-\mathrm{HNO}_{3}-\mathrm{H}_{2} \mathrm{SO}_{4}$ water system, conditions can arise where the availability of $\mathrm{NH}_{3}$ limits the formation of aerosol $\mathrm{NH}_{4} \mathrm{NO}_{3}$. Here, subsequent reductions of airborne aerosol $\mathrm{SO}_{4}$, by tying up less $\mathrm{NH}_{3}$ as aerosol $\left(\mathrm{NH}_{4}\right)_{2} \mathrm{SO}_{4}$, may make more $\mathrm{NH}_{3}$ available to react with $\mathrm{HNO}_{3}$, increasing the level of aerosol $\mathrm{NH}_{4} \mathrm{NO}_{3}$. Although atmospheric $\mathrm{NH}_{3}$ concentration is not monitored in CASTNET, when the index, $\mathrm{RNO}_{3}$ (i.e., the ratio of atmospheric aerosol $\mathrm{NO}_{3}$ to $\mathrm{OxN}$ concentrations), is less than 0.7 , then formation of aerosol $\mathrm{NH}_{4} \mathrm{NO}_{3}$ may be $\mathrm{NH}_{3}$-limited (Blanchard et al., 2000). Using this index and focusing on non-summer season results to minimize the impacts of sampling artifacts (see Sect. 2), results in Table 3 suggest that $\mathrm{NH}_{3}$-limited conditions are present in the east but are weakest in winter and year-round in the midwest.

Significant P1-to-P2-to-P3-to-P4 increases in $\mathrm{RNO}_{3}$ occur in the east. Regionally, the numerically largest absolute and relative P1-to-P4 increases occur in the northeast (40\%), with smaller increases in the south $(28 \%)$, midwest $(10 \%)$, and east (22\%). Seasonally, the numerically largest absolute increases occur in winter. The numerically largest regional $\mathrm{P} 1$ winter $\mathrm{RNO}_{3}$ value $(0.70)$ occurs in the midwest, and this value increases significantly by $12 \%$ to 0.78 in $\mathrm{P} 4$. These results suggest that between $\mathrm{P} 1$ and $\mathrm{P} 4, \mathrm{NH}_{3}$ availability has increased in the east and the formation of aerosol $\mathrm{NO}_{3}$ has become less $\mathrm{NH}_{3}$-limited. The least $\mathrm{NH}_{3}$-limited situation appears to occur in winter in the midwest (with its strong sources of $\mathrm{NH}_{3}$ ). In addition, the relative amount of monitored OxN present as $\mathrm{HNO}_{3}, \mathrm{RHNO}_{3}$ (i.e., 1- $\mathrm{RNO}_{3}$ ), diminished significantly in the east between P1 (0.66) and P4 (0.59) (Sect. 3.3).

For a chemical system initially containing $\mathrm{NH}_{3}, \mathrm{HNO}_{3}$, $\mathrm{H}_{2} \mathrm{SO}_{4}$, and water, an index of the extent to which ambient sulfuric acid $\left(\mathrm{H}_{2} \mathrm{SO}_{4}\right)$ has been neutralized by atmospheric $\mathrm{NH}_{3}$ may be defined as the neutralization index (NI). Correcting for the presence aerosol $\mathrm{NH}_{4} \mathrm{NO}_{3}$, it may be computed as $\left(\left[\mathrm{NH}_{4}\right]-\left[\mathrm{NO}_{3}\right]\right) / 2\left[\mathrm{SO}_{4}\right]$ (Blanchard and Hidy, 2005). The value of NI can range between zero (un-neutralized $\left.\mathrm{H}_{2} \mathrm{SO}_{4}\right)$ and unity $(100 \%$ neutralization to $\left.\left(\mathrm{NH}_{4}\right)_{2} \mathrm{SO}_{4}\right)$. As shown in Table 3, NI averages $\approx 0.8$ in the east. Calculation of NI assumes that aerosol $\mathrm{NO}_{3}$ is present as fine aerosol $\mathrm{NH}_{4} \mathrm{NO}_{3}$ and neglects the possible presence other forms of aerosol $\mathrm{NO}_{3}$ (e.g., from large particle sea/road salt or soil/mineral dust). As suggested in Sect. 2, sampling artifacts may provide biases in opposing directions for aerosol $\mathrm{NO}_{3}$. Their impacts are expected to be minimal during cooler season sampling at non-marine sites. Although small (but significant) P1-to-P4 increases in NI occur in the east, large increases occur in summer in the northeast $(15 \%)$ and south $(18 \%)$, while small non-significant decreases occur in winter. These results suggest that between $\mathrm{P} 1$ and $\mathrm{P} 4$ an acidic aerosol is approaching neutralization due to increased relative availability of $\mathrm{NH}_{3}$ associated with reductions of aerosol $\mathrm{SO}_{4}$ concentration. These findings are consistent with those noted in previous paragraphs and with those for P1-to-P3 (SS07b), indicating that conditions in the east are becoming less $\mathrm{NH}_{3}$-limited.

Modeling efforts have indicated that nonlinear changes in $\mathrm{PM}_{2.5}$ concentrations can occur in response to reductions in atmospheric aerosol $\mathrm{SO}_{4}$ concentrations in the east (Ansari and Pandis, 1998; West et al., 1999; Blanchard and Hidy, 2005). Under $\mathrm{NH}_{3}$-limited conditions Blanchard et al. (2000) suggest that reduction of aerosol $\mathrm{SO}_{4}$ concentrations can increase aerosol $\mathrm{NO}_{3}$ concentrations by amounts that exceed the aerosol $\mathrm{SO}_{4}$ reductions on a mass basis, and that reductions of ambient oxidized nitrogen concentrations (that may accompany reductions in $\mathrm{NO}_{\mathrm{x}}$ emissions) may not reduce aerosol $\mathrm{NO}_{3}$ concentrations. These modeling studies suggest that for the $\mathrm{NH}_{3}-\mathrm{HNO}_{3}-\mathrm{H}_{2} \mathrm{SO}_{4}$ water system, constituent concentration and temperature conditions can exist in the east so that when the atmospheric aerosol $\mathrm{SO}_{4}$ concentration is reduced, the corresponding change in $\mathrm{PM}_{2.5}$ concentration can be influenced nonlinearly. At low temperatures, aerosol 
a

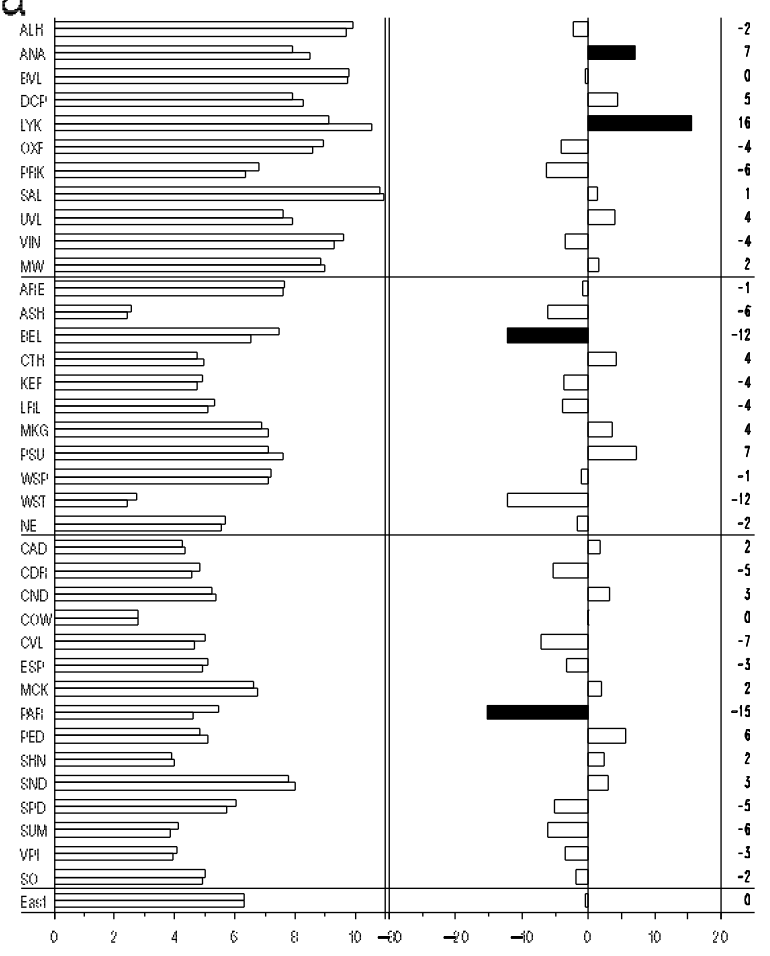

b

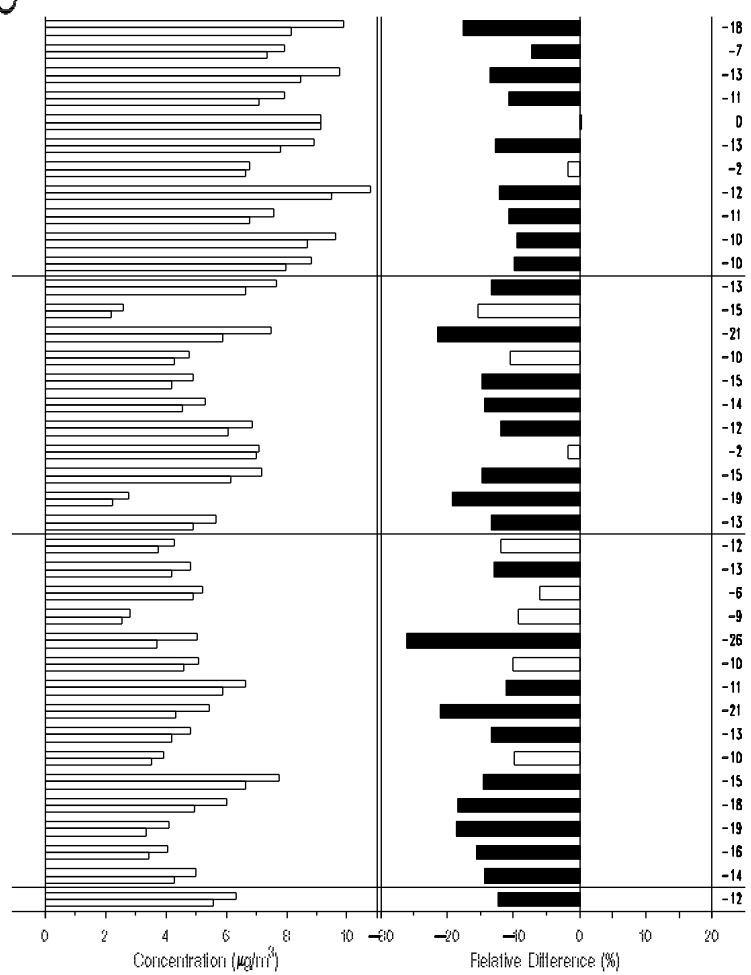

C

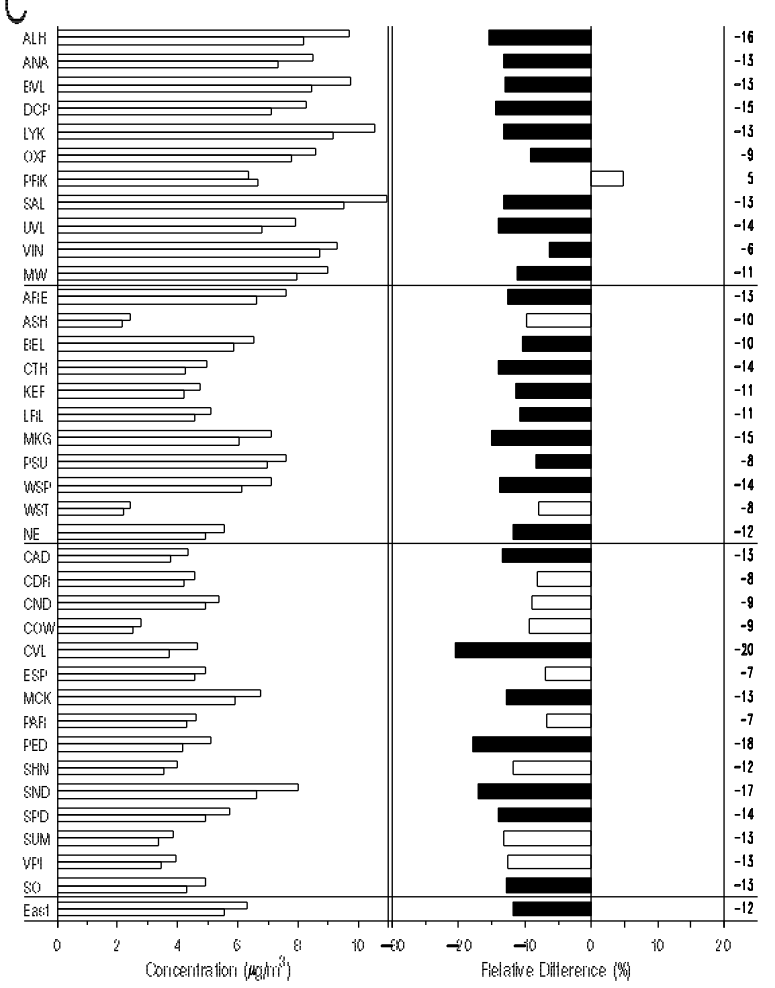

Figure 8. Site- and region-specific wintertime CASTNET PM concentration $\left(\mu \mathrm{g} \mathrm{m}^{-3}\right.$, left - upper bar: early period; and lower bar: later period) and period-to-period relative changes (\%, right and bottom) for (a) P1-to-P3, W; (b) P1-to-P4, W; and (c) P3-to-P4, W. Significance level of period-to-period relative changes indicated by bar fill: black, $p \leq 0.05$; and open, $p>0.05$ (NS). Sites are identified by region in the same order as shown in Table 1. 
$\mathrm{NH}_{4} \mathrm{NO}_{3}$ will likely form when $\mathrm{NH}_{3}$ is made available from a reduction in atmospheric aerosol $\mathrm{SO}_{4}$ concentration, and negative to nonlinear responses ranging from -0.3 to $<1.0$ $\left.\left(\Delta \mu \mathrm{g} \mathrm{PM}_{2.5} / \Delta \mu \mathrm{g} \mathrm{SO}\right)_{4}\right)$ can result (Ansari and Pandis, 1998; West et al., 1999). Using model estimates, West et al. (1999) have predicted that these conditions would exist most commonly in winter in the midwest and would be uncommon in summer in the east. A 2009 field study (Stanier et al., 2012) has focused on wintertime episodes of elevated aerosol $\mathrm{NO}_{3}$ concentrations in the midwest (Wisconsin).

Ratios of P1-to-P4 absolute changes in CASTNET PM concentrations to corresponding changes in aerosol $\mathrm{SO}_{4}-$ $\Delta\left(\mu \mathrm{gCASTNET} \mathrm{PMm} \mathrm{m}^{-3}\right) / \Delta\left(\mu \mathrm{g} \mathrm{SO}_{4} \mathrm{~m}^{-3}\right)$ - have been determined. Year round, these ratios are of similar magnitude, numerically falling between 1.2 and 1.6, both for the east and for each regional grouping of sites. Seasonally in the east, these ratios range between 1.3 and 1.5 in spring, summer, and fall. However, in winter they are numerically close to 1.0 in the east and each region. This represents a change from P1to-P3 behavior, where the wintertime ratio averaged close to 0.0 in the east, and in the midwest was negative and accompanied by a numerical increase in CASTNET PM (SS07b). Wintertime numerical increases in CASTNET PM occurred at 15 sites between P1 and P3, but at only one site between $\mathrm{P} 1$ and P4 and at only one site between P3 and P4 (Fig. 8). This wintertime behavior of CASTNET PM appears to be driven largely by that of aerosol $\mathrm{NO}_{3}$ (Fig. 7). These findings suggest that additional $\mathrm{P} 3$-to-P4 reductions in emissions of $\mathrm{SO}_{2}$, and especially $\mathrm{NO}_{\mathrm{x}}$, have made progress in altering the chemical regime of the wintertime eastern US atmosphere so that future emissions reductions and their resulting reductions in aerosol concentrations may no longer be accompanied by sub-linear changes (or actual increases) in CASTNET PM.

\subsection{Comparison with emissions estimates}

Emissions estimates for $\mathrm{SO}_{2}$ and $\mathrm{NO}_{\mathrm{x}}$ (Xing et al., 2013) have been aggregated to regional levels and have been used to determine current mean estimates of P1, P2, P3 and P4 emissions as well as period-to-period absolute and relative changes in regional $\mathrm{SO}_{2}$ and $\mathrm{NO}_{\mathrm{x}}$ emissions in the east (see Sect. 2.1 for identification of the states composing each geographical region). Although current P1, P2, and P3 emissions estimates have been updated and may be slightly different compared to those reported in SS07b, using the same current version permits period-to-period comparisons to be made using consistent emissions estimates. In addition, subsequent analyses, repeated using emissions estimates (US EPA, National Emissions Inventory, Emissions Factor and Inventory Group, Office of Air Quality Planning and Standards, Research Triangle Park, NC, http://www.epa.gov/ttn/chief/net/ 2008inventory.html, accessed 02/12/13), yielded reasonably consistent findings with no appreciable changes to conclusions.
Continuous emissions monitors used on most EGUs provide reasonably accurate estimates of $\mathrm{SO}_{2}$ and $\mathrm{NO}_{\mathrm{x}}$ emissions from these sources. Although EGUs are the main source of $\mathrm{SO}_{2}$ emissions; EGUs, on-road, and off-road sources contribute substantially to total $\mathrm{NO}_{\mathrm{x}}$ emissions. Uncertainty in on-road and other non-EGU $\mathrm{NO}_{\mathrm{x}}$ emissions make estimates of total $\mathrm{NO}_{\mathrm{x}}$ emissions more uncertain than those for $\mathrm{SO}_{2}$ (Butler et al., 2005; Parrish, 2006; Blanchard et al., 2013).

Estimates of both P1 and P4 emissions density are shown in Table 4 in the same units as deposition. For example, the $\mathrm{P} 1$ emissions density for $\mathrm{SO}_{2}$ is over $60 \%$ higher in the midwest, with its EGU sources in the Ohio Valley, than in the south (i.e., 51 vs. $31 \mathrm{~kg} \mathrm{~S}\left(\right.$ ha yr) ${ }^{-1}$ ). P1-to-P4 relative change results for regional $\mathrm{SO}_{2}$ and $\mathrm{NO}_{\mathrm{x}}$ emissions (Fig. 3) are also shown in Table 4 with corresponding values for selected measures of atmospheric concentration and total deposition from Fig. 4.

\subsubsection{Oxidized sulfur species}

The fairly universal reductions in the S-related concentration and deposition metrics noted previously in Sect. 3.2 largely reflect the year-round $\mathrm{SO}_{2}$ emissions controls that have been continuing since 1995 (Fig. 1). Reductions in $\mathrm{SO}_{2}$ emissions in the east have led to significant reductions in monitored $\mathrm{SO}_{2}$ concentrations, i.e., P1-to-P2 (21 vs. $27 \%$ ), P1to-P3 (35 vs. $33 \%$ ), P1-to-P4 (50 vs. $48 \%$ ), and P3-to-P4 (23 vs. $23 \%$ ). Reductions in $\mathrm{SO}_{2}$ emissions in the east have led to significant, but smaller, reductions in aerosol $\mathrm{SO}_{4}$ concentrations, i.e., P1-to-P2 (21 vs. $12 \%$ ), P1-to-P3 (35 vs. $21 \%$ ), P1-to-P4 (50 vs. $33 \%$ ), and P3-to-P4 (23 vs. $15 \%$ ).

As indicated in Table 4, the P1-to-P4 relative reductions in $\mathrm{SO}_{2}$ emissions in the east (50\%) are in closer numerical agreement with reductions of atmospheric concentration of the monitored primary pollutant, $\mathrm{SO}_{2}(48 \%)$, than the monitored reaction product, aerosol $\mathrm{SO}_{4}(33 \%)$. Ratios of the $\mathrm{P} 1$-to-P4 relative change in monitored concentration to emissions estimates for $\mathrm{SO}_{2}$ in the east are approximately 0.97 for $\mathrm{SO}_{2}$ and 0.66 for aerosol $\mathrm{SO}_{4}$, with similar findings for P3-to-P4 comparisons. Relative P1-to-P4 comparisons also show significant reductions for $\mathrm{SO}_{2}$ emissions $(50 \%)$ and dry S (49\%). These results suggest that at the current scale, the responses of both atmospheric $\mathrm{SO}_{2}$ concentration and dry deposition to changes in $\mathrm{SO}_{2}$ emissions are close to $1: 1$; however, the relationship appears to be less than $1: 1$ for aerosol $\mathrm{SO}_{4}$. In addition, as indicated here and in Sect. 4.1, the relationship between changes in the concentration of the reaction product, aerosol $\mathrm{SO}_{4}$, and that of the primary pollutant, $\mathrm{SO}_{2}$, appears to be less than $1: 1$.

These findings are generally consistent with earlier findings for P1-to-P3 comparisons (SS07b) as well as those of those of others. Butler et al. (2001) reported slightly less than a $1: 1$ relationship between changes in downwind atmospheric $\mathrm{S}$ concentration and changes in $\mathrm{SO}_{2}$ emissions 
Table 4. $\mathrm{P} 1$ and $\mathrm{P} 4$ emissions density and $\mathrm{P} 1$-to-P4 relative changes $(\%)$ in oxidized sulfur and nitrogen emissions, atmospheric concentration, and dry, wet, and total deposition.

\begin{tabular}{|c|c|c|c|c|c|c|c|c|c|}
\hline \multirow[b]{2}{*}{ Region } & \multicolumn{2}{|r|}{ Emissions } & \multicolumn{3}{|c|}{$\begin{array}{l}\text { P1-to-P4 Change in } \\
\text { atmospheric concentration }\end{array}$} & \multicolumn{4}{|c|}{$\begin{array}{l}\text { P1-to-P4 Change in } \\
\text { deposition }\end{array}$} \\
\hline & $\rho_{1}^{\mathrm{a}}\left(\rho_{4}\right)$ & P1-to-P4 Change & $\mathrm{SO}_{2}$ & $\mathrm{SO}_{4}$ & S & Dry $S$ & Wet S & Total S & Wet $\mathrm{H}^{+}$ \\
\hline $\mathrm{E}$ & 38 (19) & -50 & -48 & -33 & -45 & -49 & -32 & -40 & -47 \\
\hline MW & $51(23)$ & -55 & -51 & -33 & -47 & -48 & -36 & -42 & -56 \\
\hline $\mathrm{NE}$ & 36 (19) & -47 & -48 & -34 & -45 & -51 & -30 & -40 & -44 \\
\hline \multirow[t]{2}{*}{ SO } & 31 (16) & -47 & -47 & -32 & -42 & -49 & -31 & -39 & -46 \\
\hline & $\rho_{1}^{\mathrm{b}}\left(\rho_{4}\right)$ & P1-to-P4 Change & $\mathrm{HNO}_{3}$ & $\mathrm{NO}_{3}$ & $\mathrm{OxN}$ as $\mathrm{N}$ & Dry OxN & Wet OxN & Total OxN & Wet $\mathrm{H}^{+}$ \\
\hline $\mathrm{E}$ & $19(11)$ & -42 & -34 & -8 & -24 & -34 & -29 & -31 & -47 \\
\hline MW & $21(12)$ & -43 & -30 & -13 & -21 & -27 & -28 & -28 & -56 \\
\hline $\mathrm{NE}$ & $23(12)$ & -46 & -39 & $-1^{\mathrm{c}}$ & -29 & -40 & -34 & -36 & -44 \\
\hline SO & $17(10)$ & -39 & -32 & $-2^{c}$ & -24 & -34 & -25 & -29 & -46 \\
\hline
\end{tabular}

${ }^{\text {a }} \mathrm{SO}_{2}$ emissions density in units of $\mathrm{kgS}$ (ha yr) ${ }^{-1}$

${ }^{\mathrm{b}} \mathrm{NO}_{\mathrm{x}}$ emissions density in units of $\mathrm{kgN}(\text { ha } \mathrm{yr})^{-1}$

c $p>0.05$, NS.

from specific upwind source regions. Blanchard et al. (2013) found southeastern US trends of $\mathrm{SO}_{2}$ ambient concentrations and emissions to be decreasing with a qualitative $1: 1$ proportionality, while the relationship for trends of ambient aerosol $\mathrm{SO}_{4}$ concentrations and $\mathrm{SO}_{2}$ emissions were decreasing but at less than $1: 1$ proportionality. Hand et al. (2012) reported linear relationships between aerosol $\mathrm{SO}_{4}$ concentrations and $\mathrm{SO}_{2}$ emissions that depended on location in the US, with aerosol $\mathrm{SO}_{4}$ concentration showing the weakest response to $\mathrm{SO}_{2}$ emissions in a region in the eastern US roughly included in the currently defined source region, midwest, and northeast.

Atmospheric pollutants are transported and transformed by physical and chemical processes between their release and deposition. For example, gaseous $\mathrm{SO}_{2}$ may be transformed into aerosol $\mathrm{SO}_{4}$ by several pathways, including gasphase oxidation by the hydroxyl radical and, after dissolution in water droplets, via oxidation by hydrogen peroxide and $\mathrm{O}_{3}$ (McHenry and Dennis, 1994). As $\mathrm{SO}_{4}$-containing aqueous particles undergo atmospheric processing (i.e., evaporation and dissolution), there is a close linkage between the secondary pollutant's atmospheric concentration and wet removal (i.e., they have similar atmospheric timescales). Although $\mathrm{SO}_{4}$ is then subject to both dry and wet deposition, the latter dominates (e.g., east $\mathrm{P} 4$ wet $\mathrm{S}$ is $5.2 \mathrm{~kg} \mathrm{~S}$ (ha yr) ${ }^{-1}$ vs. $0.4 \mathrm{~kg} \mathrm{~S}(\text { ha yr) })^{-1}$ for dry $\mathrm{SO}_{4}$; Table 3 ). This may explain the relatively good agreement between P1-to-P4 (33\%) and $\mathrm{P} 3$-to-P4 $(15 \%)$ reductions in the atmospheric concentration of secondary pollutant, aerosol $\mathrm{SO}_{4}$, and corresponding changes (32 and $13 \%$ ) in wet S.

Since atmospheric $\mathrm{S}$ concentration is comprised of $\mathrm{SO}_{2}$ and aerosol $\mathrm{SO}_{4}$, reductions of $\mathrm{SO}_{2}$ emissions may be expected to yield responses of atmospheric $\mathrm{S}$ concentration and total $\mathrm{S}$ that fall between corresponding values for $\mathrm{SO}_{2}$ and aerosol $\mathrm{SO}_{4}$. This is confirmed in Table 4 where reductions in $\mathrm{SO}_{2}$ emissions in the east correspond to significant reductions of both atmospheric $\mathrm{S}$ concentration (i.e., P1-to-P4, 50 vs. $45 \%$ ) and total S (i.e., P1-to-P4, 50 vs. $40 \%$ ).

For a pollutant where all primary and secondary species are monitored (e.g., S), neglecting other sources and sinks, emissions density may be compared with total deposition to construct rough budgets for oxidized sulfur, revealing insights about the relative amounts of the species deposited and exported. Model predictions for North America (Wojcik and Chang, 1997) suggest approximately $50 \%$ of S emissions are deposited. In the current study, P1, P2, P3 and $\mathrm{P} 4 \mathrm{~S}$ emissions densities in the east are $38,30,25$, and $19 \mathrm{~kg} \mathrm{~S}$ (ha yr) ${ }^{-1}$, while corresponding total $\mathrm{S}$ values are 15 , 12,11 and $8.7 \mathrm{~kg} \mathrm{~S}$ (ha yr) ${ }^{-1}$. Between P1 and P4 the relative amount of deposited $\mathrm{S}$ emissions increased by $\approx 8 \%$ from 39 to $47 \%$, and the net exported S emissions decreased by a corresponding amount from $61 \%$ to $53 \%$. Thus, the strong P1to-P4 $\mathrm{SO}_{2}$ emissions controls are accompanied by increased apparent conversion of $\mathrm{SO}_{2}$ to aerosol $\mathrm{SO}_{4}$ (Sect. 4.1), possible increased relative contribution of wet vs. dry deposition (Sect. 4.2), increased fraction of deposited S emissions, and decreased fraction of exported $\mathrm{S}$ emissions.

\subsubsection{Oxidized nitrogen species}

Changes in pollutant emissions generally lead to more direct changes in the atmospheric concentration of primary rather than secondary pollutant species, where emissions as well as variable meteorological and atmospheric chemical processes are influential. In contrast to oxidized sulfur species, where the behaviors of both primary and secondary pollutants are 
monitored, the atmospheric concentration and deposition of only a few of the secondary oxidized nitrogen species (i.e., $\mathrm{HNO}_{3}$, aerosol $\mathrm{NO}_{3}$ and aqueous $\mathrm{NO}_{3}$ ion) are monitored in the current study. This likely propagates more uncertainty into comparisons with primary $\mathrm{NO}_{\mathrm{x}}$ emissions than is present in earlier comparisons with $\mathrm{SO}_{2}$ emissions.

Recent aggressive $\mathrm{NO}_{\mathrm{x}}$ emissions controls in the east have led to significant reductions in concentrations of the important secondary oxidized nitrogen pollutant, $\mathrm{HNO}_{3}$, i.e., $\mathrm{P} 1$ to-P2 (6\% vs. NS), P1-to-P3 (22 vs. 13\%), P1-to-P4 (42 vs. $34 \%$ ), and P3-to-P4 (25 vs. $24 \%$ ). The nonlinear behavior of aerosol $\mathrm{NO}_{3}$ concentration noted in Sect. 4.3 is apparent in many comparisons of reductions in $\mathrm{NO}_{\mathrm{x}}$ emissions with aerosol $\mathrm{NO}_{3}$ behavior but appears to be diminishing with time (i.e., P1-to-P2 (6 vs. $-5 \%)$, P1-to-P3 ( 22 vs. $-11 \%$ ), P1-to-P4 (42 vs. $8 \%$ ), and P3-to-P4 (25 vs. $17 \%$ ), where the minus sign indicates increase).

The numerically largest $\mathrm{P} 1$-to-P4 relative reductions in estimated $\mathrm{NO}_{\mathrm{x}}$ emissions (46\%), year-round atmospheric concentrations of $\mathrm{HNO}_{3}(39 \%)$ and OxN (29\%), dry OxN $(40 \%)$, wet $\mathrm{OxN}(34 \%)$, total OxN (36\%), and summertime total OxN (42\%) occur in the northeast, the region with the largest P1 emissions density (Table 4). As noted in Sect. 3.3, numerical maximum absolute and relative seasonal reductions in atmospheric $\mathrm{HNO}_{3}$ concentrations also occur in summer, with largest reductions in the northeast. These changes are likely reflecting contributions from year-round control of $\mathrm{NO}_{\mathrm{x}}$ emissions by Phases I and II of the CAAA and continuing mobile source controls. However, the timing (e.g., changes most apparent in summer with peak concentrations moving from summer to spring) and the location (i.e., strongest reductions in northeast) suggest that major contributors are summertime $\mathrm{NO}_{\mathrm{x}}$ emissions control activities instituted under OTC, SIP Call, BTP and CAIR that focused on EGU sources in the eastern states.

As noted above and in SS07b, the $22 \%$ reduction in the east of P1-to-P3 $\mathrm{NO}_{\mathrm{x}}$ emissions is associated with a $13 \%$ reduction in $\mathrm{HNO}_{3}$ concentration, an $8 \%$ reduction in dry $\mathrm{OxN}$, a $12 \%$ reduction in wet $\mathrm{OxN}$, an $11 \%$ reduction in total $\mathrm{OxN}$, but an $11 \%$ increase in aerosol $\mathrm{NO}_{3}$ concentration. Here, the ratio of P1-to-P3 relative changes of monitored $\mathrm{HNO}_{3}$ concentration to those of estimated $\mathrm{NO}_{\mathrm{x}}$ emissions is 0.6 , qualitatively consistent with the value of 0.7 found by Butler et al. (2005) for a similar time period (1991-2001). Recent aggressive $\mathrm{NO}_{\mathrm{x}}$ emissions controls, however, appear to have altered these relationships. As indicated in Table 4, the $42 \%$ P1-to-P4 reduction of $\mathrm{NO}_{\mathrm{x}}$ emissions in the east corresponds to $34 \%$ reductions in $\mathrm{HNO}_{3}$ concentration and dry $\mathrm{OxN}$, a $29 \%$ reduction in wet $\mathrm{OxN}$, a $31 \%$ reduction in total $\mathrm{OxN}$, and an $8 \%$ reduction in aerosol $\mathrm{NO}_{3}$ concentration. Here, the ratio of P1-to-P4 relative changes of monitored $\mathrm{HNO}_{3}$ concentration and dry $\mathrm{OxN}$ to those of estimated $\mathrm{NO}_{\mathrm{x}}$ emissions is 0.8 , but the corresponding ratio for aerosol $\mathrm{NO}_{3}$ concentration is 0.2 . These findings are consistent with the recent observations in the southeastern US of decreasing
1999-2010 trends of $\mathrm{NO}_{\mathrm{y}}$ concentration that exceed those for aerosol $\mathrm{NO}_{3}$ concentration and are qualitatively proportional to decreasing $\mathrm{NO}_{\mathrm{x}}$ emissions trends (Blanchard et al., 2013).

In the east, the $42 \%$ P1-to- $\mathrm{P} 4$ reduction of $\mathrm{NO}_{\mathrm{x}}$ emissions also corresponds to significant reductions of deposition, i.e., dry $\mathrm{HNO}_{3}(35 \%)$, dry OxN (34\%), wet OxN (29\%) and total OxN (31\%). The relatively good agreement between the P1-to-P4 changes in the atmospheric concentration of $\mathrm{HNO}_{3}$, dry $\mathrm{HNO}_{3}$, and dry OxN can be explained by the high $\mathrm{RFHNO}_{3}$ (Sect. 3.3; Table 3). The favorable comparison with wet $\mathrm{OxN}$ may be related to the high relative abundance and high water solubility of $\mathrm{HNO}_{3}$ in comparison to other $\mathrm{NO}_{\mathrm{y}}$ species in the atmosphere. It could also be related to a possible high correlation of atmospheric concentration of $\mathrm{HNO}_{3}$ with those of other airborne $\mathrm{NO}_{\mathrm{x}}$ reaction products that are removed from the atmosphere and measured as aqueous $\mathrm{NO}_{3}$ ion in precipitation.

Model predictions for the continental US (Zhang et al., 2012) suggest approximately $63 \%$ of $2006-2008 \mathrm{NO}_{\mathrm{x}}$ emissions are deposited as $\mathrm{NO}_{\mathrm{y}}$ and $45 \%$ as OxN. In the current study, none of the primary $\mathrm{NO}_{\mathrm{x}}$ species and only two of the secondary species $\left(\mathrm{HNO}_{3}\right.$ and aerosol $\left.\mathrm{NO}_{3}\right)$ are monitored. Nevertheless, neglecting other sources and sinks, it is possible to compare $\mathrm{NO}_{\mathrm{x}}$ emissions density with total OxN deposition to determine the fraction of $\mathrm{NO}_{\mathrm{x}}$ emissions deposited as OxN. Using the approach described in Sect. 4.4.1 for oxidized sulfur, approximately $30 \%$ of the $\mathrm{NO}_{\mathrm{x}}$ emissions appear to be deposited as $\mathrm{OxN}$ during the study period. Although there are hints of a numerically small P1to-P4 increase in the relative amount of $\mathrm{NO}_{\mathrm{x}}$ emissions deposited as $\mathrm{OxN}$, the large relative uncertainty associated with $\mathrm{NO}_{\mathrm{x}}$ emissions estimates (Sect. 4.4) precludes a conclusion in this instance. Nevertheless, the strong P1-to-P4 reduction of $\mathrm{NO}_{\mathrm{x}}$ emissions (42\%) is accompanied by reduced atmospheric concentration of $\mathrm{HNO}_{3}$ and $\mathrm{OxN}$ as well as $\mathrm{RHNO}_{3}$ (Sects. 3.3 and 4.3), and by increased relative contribution of wet OxN vs. dry OxN (Sect. 4.2).

\subsubsection{Wet $\mathrm{H}^{+}$}

In the east the $\mathrm{P} 1$-to- $\mathrm{P} 2$ reductions in estimated emissions are $21 \%$ for $\mathrm{SO}_{2}$ and $6 \%$ for $\mathrm{NO}_{\mathrm{x}}$. Concurrent changes in deposition include reductions of $17 \%$ for wet S, $3 \%$ (NS) for both wet $\mathrm{OxN}$ and wet $\mathrm{NH}_{4}$, and $24 \%$ for wet $\mathrm{H}^{+}$(not shown). In the absence of sizeable $\mathrm{P} 1$-to-P2 changes in $\mathrm{NO}_{\mathrm{x}}$ emissions, it appears that $\mathrm{P} 1$-to- $\mathrm{P} 2$ reductions in $\mathrm{SO}_{2}$ emissions played a major role in the resulting reductions of both wet $\mathrm{S}$ and wet $\mathrm{H}^{+}$in the east. P1-to-P3 reductions in estimated $\mathrm{SO}_{2}$ and $\mathrm{NO}_{\mathrm{x}}$ emissions are 35 and $22 \%$, with corresponding reductions of $22 \%$ in wet $\mathrm{S}, 12 \%$ in wet OxN, no change in wet $\mathrm{NH}_{4}$, and $34 \%$ in wet $\mathrm{H}^{+}$(SS07b). For P1-to-P4, reductions in estimated $\mathrm{SO}_{2}$ and $\mathrm{NO}_{\mathrm{x}}$ emissions are 50 and $42 \%$, and corresponding reductions are $32 \%$ for wet $\mathrm{S}, 29 \%$ for wet $\mathrm{OxN}, 9 \%$ for wet $\mathrm{NH}_{4}$, and $47 \%$ for wet $\mathrm{H}^{+}$(Fig. 4). Respective P3-to-P4 reductions in estimated $\mathrm{SO}_{2}$ and $\mathrm{NO}_{\mathrm{x}}$ 
emissions are 23 and $25 \%$, with corresponding reductions of $13 \%$ in wet $\mathrm{S}, 19 \%$ in wet $\mathrm{OxN}, 9 \%$ in wet $\mathrm{NH}_{4}$, and $20 \%$ in wet $\mathrm{H}^{+}$.

Changes in wet $\mathrm{NH}_{4}$ between $\mathrm{P} 1$ and $\mathrm{P} 3$ are generally small, variable, and non-significant. Although significant P1-to-P4 reductions in wet $\mathrm{NH}_{4}$ are also relatively small $(\approx 9 \%)$, they occur mainly between $\mathrm{P} 3$ and $\mathrm{P} 4$. In the east between $\mathrm{P} 1$ and $\mathrm{P} 4$, with the continuing reduction in $\mathrm{SO}_{2}$ and $\mathrm{NO}_{\mathrm{x}}$ emissions, the fraction of wet $\mathrm{S}$ plus wet $\mathrm{OxN}$ that could be neutralized on an equivalent basis by wet $\mathrm{NH}_{4}$ increased by approximately $8 \%$ from $25 \%$ in P1, to $33 \%$ in P4.

Neglecting the relatively small temporal impacts of wet $\mathrm{NH}_{4}$ (above) and those of other wet cations, examination of the behavior of wet $\mathrm{S}$ plus wet $\mathrm{OxN}$ (on an equivalent basis) permits rough estimation of the relative contributions of reductions in $\mathrm{SO}_{2}$ and $\mathrm{NO}_{\mathrm{x}}$ emissions to corresponding reductions in wet $\mathrm{H}^{+}$. Year-round in the east, P1-to-P3 reductions in wet $\mathrm{S}$ contribute most $(\approx 80 \%)$ of the relative reduction of wet $\mathrm{S}$ plus wet $\mathrm{OxN}$ associated with reduced wet $\mathrm{H}^{+}$. Between $\mathrm{P} 1$ and $\mathrm{P} 4$, this relative contribution diminishes (to $\approx 70 \%$ ), and between $\mathrm{P} 3$ and $\mathrm{P} 4$ the relative contribution of wet $\mathrm{S}(\approx 55 \%)$ approaches that of wet $\mathrm{OxN}$. This finding illustrates the impact of recent aggressive $\mathrm{NO}_{\mathrm{x}}$ emissions controls along with continuing $\mathrm{SO}_{2}$ controls on wet $\mathrm{H}^{+}$and the increasing relative contribution of reductions in $\mathrm{NO}_{\mathrm{x}}$ emissions to the significant and substantial reduction of wet $\mathrm{H}^{+}$ in the eastern US.

\section{Summary, conclusions, and recommendations}

Data collected in the eastern US between 1990 and 2009 at 34 CASTNET dry monitoring sites and paired NADP wet monitoring sites are examined. A major objective is to evaluate the monitored air quality impacts occurring between 1990 and 2009 that are associated with concurrent legislatively mandated changes in emissions. Four 5-year periods (P1, P2, P3, and P4) are considered. Period-to-period changes in selected pollutant metrics are examined, focusing on P1-to-P4 changes. Data are composed from reported weekly measurements into period-site-season means. These means, computed for atmospheric concentration, Vd, PR, and dry, wet, and total deposition, are used to examine differences between 5-year periods for seasons, sites, and predefined regional groupings of sites. The approach to data analysis uses the mean squared error derived from analysis of variance of mean estimates for each pollutant metric to examine differences in the monitoring data and metrics derived from them.

Estimated $\mathrm{SO}_{2}$ emissions for $\mathrm{P} 4$ are $50 \%$ lower than for $\mathrm{P} 1$. This is accompanied in each regional site grouping in each season and over all seasons by significant reductions in atmospheric $\mathrm{SO}_{2}$ concentration and dry deposition, atmospheric aerosol $\mathrm{SO}_{4}$ concentration, atmospheric $\mathrm{S}$ concentration and dry deposition, wet $\mathrm{S}$ (except in winter), and total
S. For atmospheric $\mathrm{SO}_{2}$ concentration and dry deposition, the numerically largest significant seasonal absolute reductions usually occur in winter when atmospheric $\mathrm{SO}_{2}$ concentration is at its seasonal maximum. Analogous behavior occurs in summer for atmospheric aerosol $\mathrm{SO}_{4}$ concentration, dry $\mathrm{SO}_{4}$, and wet $\mathrm{S}$, when atmospheric aerosol $\mathrm{SO}_{4}$ concentration is at its seasonal maximum. In the east, significant reductions of $48 \%, 33 \%$, and $45 \%$ for atmospheric concentration of $\mathrm{SO}_{2}$, aerosol $\mathrm{SO}_{4}$ and atmospheric $\mathrm{S}$, and of $49 \%, 32 \%$, and $40 \%$ for dry, wet, and total deposition of atmospheric $\mathrm{S}$ are associated with the $50 \%$ P1-to-P4 reduction in estimated $\mathrm{SO}_{2}$ emissions. Consistent with earlier findings (SS07b), results suggest that at the current scale, the responses of both atmospheric $\mathrm{SO}_{2}$ concentration and dry deposition to changes in $\mathrm{SO}_{2}$ emissions are close to $1: 1$ and the relationship between changes in the concentration of the secondary reaction product, aerosol $\mathrm{SO}_{4}$, and that of the primary pollutant, $\mathrm{SO}_{2}$, appears to be less than $1: 1$. The strong $\mathrm{P} 1$ to- $\mathrm{P} 4$ reduction of $\mathrm{SO}_{2}$ emissions (50\%) is accompanied by increased apparent conversion of $\mathrm{SO}_{2}$ to aerosol $\mathrm{SO}_{4}$, possible increased relative contribution of wet vs. dry deposition as a sink for $\mathrm{S}$, increased fraction of deposited $\mathrm{S}$ emissions, and decreased fraction of exported $\mathrm{S}$ emissions.

In the east, significant P1-to-P4 reductions of $34 \%, 8 \%$, and $24 \%$ for atmospheric concentration of $\mathrm{HNO}_{3}$, aerosol $\mathrm{NO}_{3}$ and $\mathrm{OxN}$, and of $34 \%, 29 \%$, and $31 \%$ for dry, wet, and total deposition of OxN are associated with the $42 \%$ reduction in estimated $\mathrm{NO}_{\mathrm{x}}$ emissions. Atmospheric aerosol $\mathrm{NO}_{3}$ concentration in the east displays a significant P1-toP3 increase (11\%), with the numerically strongest seasonal relative increase (31\%) in winter (SS07b). Recent aggressive reductions of $\mathrm{NO}_{\mathrm{X}}$ emissions have been accompanied by significant P1-to-P4 and P3-to-P4 reductions of 8 and $17 \%$ in atmospheric aerosol $\mathrm{NO}_{3}$ concentration over all seasons. In addition, the smaller P1-to-P4 wintertime increase in atmospheric aerosol $\mathrm{NO}_{3}$ concentration (i.e., $12 \%$ vs. $31 \%$ for P1-to-P3) and the P3-to-P4 wintertime reduction of $14 \%$ suggest that recent continuing $\mathrm{NO}_{\mathrm{x}}$ emissions controls are having a desirable impact. Over all seasons the numerically largest absolute and relative reductions in atmospheric $\mathrm{HNO}_{3}$ concentration and dry deposition, atmospheric $\mathrm{OxN}$ concentration and dry deposition, and total OxN generally occur in the northeast (the geographical region with the highest P1 $\mathrm{NO}_{\mathrm{x}}$ emissions density), and seasonally they tend to occur there in summer. In the east, coincident timing and location suggest that aggressive summertime $\mathrm{NO}_{\mathrm{x}}$ emissions reductions by EGU sources contributed substantially to this observed behavior. The strong P1-to-P4 reduction of $\mathrm{NO}_{\mathrm{x}}$ emissions $(42 \%)$ is accompanied by reduced atmospheric concentration of $\mathrm{HNO}_{3}$ relative to $\mathrm{OxN}$, and increased contribution of wet OxN relative to dry OxN.

Estimated annual 1990 to $2009 \mathrm{NH}_{3}$ emissions are relatively constant, ranging between 3.5 and $3.9 \mathrm{Tg} \mathrm{yr}^{-1}$ (Xing et al., 2013). However, this is not confirmed through monitoring in the current study, because only the atmospheric con- 
centration of the secondary reduced nitrogen species, aerosol $\mathrm{NH}_{4}$, is monitored. In the east, both atmospheric aerosol $\mathrm{NH}_{4}$ concentration and dry deposition show significant P1-to-P4 reductions (i.e., 26 and $22 \%$ ). The numerically largest absolute and relative reductions of atmospheric aerosol $\mathrm{NH}_{4}$ concentration and dry deposition tend to occur regionally in the midwest and northeast and seasonally in the summer (when atmospheric aerosol $\mathrm{SO}_{4}$ concentration is at its seasonal maximum and is also experiencing its largest reductions). Although showing a significant (9\%) P1-to-P4 reduction over all in the east, wet $\mathrm{NH}_{4}$ shows changes at the regional and seasonal scales that are usually not significant. Since the magnitude of wet $\mathrm{NH}_{4}$ is over seven times larger than dry deposition, the resulting reduction in total $\mathrm{NH}_{4}$ in the east (11\%) is similar to that of wet $\mathrm{NH}_{4}$.

The atmospheric concentration of $\mathrm{N}$, the sum of monitored oxidized and reduced nitrogen species, is dominated in the east by aerosol $\mathrm{NH}_{4}(\approx 67 \%)$ with smaller contributions from $\mathrm{HNO}_{3}(\approx 19 \%)$ and aerosol $\mathrm{NO}_{3}(\approx 14 \%)$. However, a high $\mathrm{Vd}$ permits $\mathrm{HNO}_{3}$ to dominate dry deposition of $\mathrm{N}$ $(\approx 75 \%)$. Both the atmospheric concentration and dry deposition of $\mathrm{N}$ show significant overall P1-to-P4 reductions (26 and $32 \%$ ) which tend to be seasonally higher in summer. Wet deposition of $\mathrm{OxN}$ and $\mathrm{NH}_{4}$ are fairly comparable, and their sum (wet $\mathrm{N}$ ) dominates total $\mathrm{N}(\approx 75 \%)$. The overall P1-to$\mathrm{P} 4$ relative reductions of wet $\mathrm{N}$ and total $\mathrm{N}$ in the east are 20 and $24 \%$, with the strongest reductions in summer and in the northeast.

In the east, a $47 \%$ reduction in wet deposition of $\mathrm{H}^{+}$ion is associated with $\mathrm{P} 1$-to-P4 reductions of 50 and $42 \%$ in $\mathrm{SO}_{2}$ and $\mathrm{NO}_{\mathrm{x}}$ emissions. The relative contributions of reduced $\mathrm{NO}_{\mathrm{x}}$ vs. $\mathrm{SO}_{2}$ emissions have increased over time, until currently (P3-to-P4), they are almost equal. Thus, recent aggressive reductions of $\mathrm{NO}_{\mathrm{x}}$ emissions along with continuing reductions of $\mathrm{SO}_{2}$ emissions appear to contribute similarly to the significant and substantial reduction of wet deposition of $\mathrm{H}^{+}$ion in the eastern US.

Although both atmospheric $\mathrm{O}_{3}$ concentration and dry deposition in the east show significant summertime P1-to-P4 reductions of $12 \%$ each, significant wintertime respective increases of 9 and $19 \%$ lead to overall significant reductions of $4 \%$ each. The numerically largest seasonal and regional reductions of both atmospheric $\mathrm{O}_{3}$ concentration and dry deposition (17 and $15 \%$ ) occur in summer in the northeast, coinciding with aggressive $\mathrm{O}_{3}$-season $\mathrm{NO}_{\mathrm{x}}$ emissions controls during $\mathrm{P} 3$ and $\mathrm{P} 4$ in the region and upwind in the east.

Although conditions in the east permitting the formation of aerosol $\mathrm{NO}_{3}$ may be limited by the availability of $\mathrm{NH}_{3}$, this appears to be diminishing over time. Increasing NI between P1 and P4 suggests that an acidic aerosol exists in the east, but is approaching neutralization due to increased relative availability of $\mathrm{NH}_{3}$ associated with reductions of aerosol $\mathrm{SO}_{4}$ concentration. Widespread wintertime numerical increases in the atmospheric concentrations of both aerosol $\mathrm{NO}_{3}$ and CASTNET PM are present between P1 and P3, despite reductions in estimated emissions of both $\mathrm{SO}_{2}$ and $\mathrm{NO}_{\mathrm{x}}$ (SS07b). Currently, this behavior appears to be largely reversed, and this reversal is associated with continuing reductions of $\mathrm{SO}_{2}$ and $\mathrm{NO}_{\mathrm{x}}$ emissions between $\mathrm{P} 3$ and $\mathrm{P} 4$. Our findings suggest that additional $\mathrm{P} 3$-to-P4 reductions in emissions of $\mathrm{SO}_{2}$, and especially $\mathrm{NO}_{\mathrm{x}}$, have made progress in altering the chemical regime of the wintertime eastern US atmosphere so that future emissions reductions and their resulting reductions in aerosol concentrations may no longer be accompanied by sub-linear changes (or actual increases) in CASTNET PM.

Monitoring data for several atmospheric species not collected by CASTNET, including $\mathrm{NH}_{3}, \mathrm{NO}, \mathrm{NO}_{2}$, PAN, other oxidized organic nitrogen species, and $\mathrm{NO}_{\mathrm{y}}$, as well as aerosol size distributions would have facilitated data analysis and interpretation. It is recommended that addition of these determinations be considered in future monitoring network upgrades. Uncertainties in estimates of many species' deposition velocities and emissions (especially $\mathrm{NO}_{\mathrm{x}}$ ) argue strongly for their continued improvement.

Acknowledgements. The US EPA, through its Office of Research and Development, funded and partially performed the research described here under contract GS-35F-4381G, BPA0775, Task Order 1521, TDD 2-5, to CSC Corporation. This manuscript has been subjected to Agency review and approved for publication.

Edited by: J. Thornton

\section{References}

Ansari, S. and Pandis, S. N.: Response of inorganic PM to precursor concentrations, Environ. Sci. Technol., 32, 2706-2714, 1998.

Baumgardner, R. E., Jr., Lavery, T. F., Rogers, C. M., and Isil, S. S.: Estimates of the atmospheric deposition of sulfur and nitrogen species: Clean Air Status and Trends Network, 1990-2000, Environ. Sci. Technol., 36, 2614-2629, 2002.

Blanchard, C. L. and Hidy, G. M.: Effects of $\mathrm{SO}_{2}$ and $\mathrm{NO}_{\mathrm{x}}$ emission reductions on $\mathrm{PM}_{2.5}$ mass concentrations in the southeastern United States, J. Air Waste Manage., 55, 265-272, 2005.

Blanchard, C. L., Roth, P. M., Tanenbaum, S. J., Ziman, S. D., and Seinfeld, J. H.: The use of ambient measurements to identify which precursor species limit aerosol nitrate formation, J. Air Waste Manage., 50, 2073-2084, 2000.

Blanchard, C. L., Hidy, G. M., Tanenbaum, S., Edgerton, E. S., and Hartsell, B. E.: The southeastern aerosol research and characterization (SEARCH) study: temporal trends in gas and PM concentrations and composition 1999-2010, J. Air Waste Manage., 63, 247-259, 2013.

Bowker, G. E., Schwede, D. B., Lear, G. G., Warren-Hicks, W. J., and Finkelstein, P. L.: Quality assurance decisions with air models: a case study of imputation of missing input data using EPA's multi-layer model, Water Air Soil Pollut., 222, 391-402, doi:10.1007/s11270-011-0832-7, 2011. 
Brook, J. R., Di- Giovanni, F., Cakmak, S., and Meyers, T. P.: Estimation of dry deposition velocity using inferential models and site-specific meteorology - uncertainty due to siting of meteorological towers, Atmos. Environ., 31, 3911-3919, 1997.

Butler, T. J., Likens, G. E., and Stunder, B. J. B.: Regional-scale impacts of Phase I of the Clean Air Act Amendments in the USA: the relation between emissions and concentration, both wet and dry, Atmos. Environ., 35, 1015-1028, 2001.

Butler, T. J., Likens, G. E., Vermeylen, F. M., and Stunder, B. J. B.: The impact of changing nitrogen oxide emissions on wet and dry nitrogen deposition in the northeastern USA, Atmos. Environ., 39, 4851-4862, 2005.

Clarke, J. F., Edgerton, E. S., and Martin, B. E.: Dry deposition calculations for the Clean Air Status and Trends Network, Atmos. Environ., 31, 3667-3678, 1997.

Finkelstein, P. L., Ellestad, T. G., Clarke, J. F., Meyers, T. P., Schwede, D. B., Hebert, E. O., and Neal, J. A.: Ozone and sulfur dioxide dry deposition to forests: observations and model evaluation, J. Geophys. Res., 105, 15365-15377, 2000.

Hand, J. L., Schichtel, B. A., Malm, W. C., and Pitchford, M. L.: Particulate sulfate ion concentration and $\mathrm{SO}_{2}$ emission trends in the United States from the early 1990s through 2010, Atmos. Chem. Phys., 12, 10353-10365, doi:10.5194/acp-1210353-2012, 2012.

Hicks, B. B.: Dry deposition to forests - on the use of data from clearings, Agric. For. Meteorol., 136, 214-221, 2006.

Hicks, B. B., Hosker, R. P., Jr., Meyers, T. P., and Womack, J. D.: Dry deposition inferential measurement techniques - I. Design and tests of a prototype meteorological and chemical system for determining dry deposition, Atmos. Environ., 25A, 2345-2359, 1991.

Holland, D. M., Caragea, P., and Smith, R. L.: Regional trends in rural sulfur concentrations, Atmos. Environ., 38, 1673-1684, 2004.

Lee, T., Yu, X.-Y., Ayers, B., Kreidenweis, S. M., Malm, W. C., and Collett Jr., J. L.: Observations of fine and coarse particle nitrate at several rural locations in the United States, Atmos. Environ. 42, 2720-2732, doi:10.1016/j.atmosenv.2007.05.016, 2008.

Lefer, B. L. and Talbot, R. W.: Summertime measurements of aerosol nitrate and ammonium at a northeastern US site, J. Geophys. Res., 106, 20365-20378, 2001.

Lovett, G. M. and Lindberg, S. E.: Atmospheric deposition and canopy interactions of nitrogen in forests, Can. J. For. Res., 23, 1603-1616, 1993.

Lu, X., Mc Elroy, M. B., Wu, G., and Nielsen, C. P.: Accelerated reduction in $\mathrm{SO}_{2}$ emissions from the U.S. power sector triggered by changing prices of natural gas, Environ. Sci. Technol., 46, 7882-7889, doi:10.1021/es301023c, 2012.

McHenry, J. N. and Dennis, R. L.: The relative importance of oxidation pathways and clouds to atmospheric ambient sulfate production as predicted by the regional acid deposition model, J. Appl. Meteorol., 33, 890-905, 1994.

Meyers, T. P., Finkelstein, P., Clarke, J., Ellestad, T. G., and Sims, P. F.: A multilayer model for inferring dry deposition using standard meteorological measurements, J. Geophys. Res., 103, 22645-22661, 1998.

Nilles, M. A., Gordon, J. D., and Schroder, L. J.: The precision of wet atmospheric deposition data from National Atmospheric Deposition Program/National Trends Network sites de- termined with collocated samplers, Atmos. Environ., 28, 11211128, 1994.

Parrish, D. D.: Critical evaluation of US on-road vehicle emission inventories, Atmos. Environ., 40, 2288-2300, 2006.

Reid, N., Misra, P. K., Bloxam, R., Yap, D., Rao, S. T., Civerolo, K., Brankov, E., and Vet, R. J.: Do we understand trends in atmospheric sulfur species?, J. Air Waste Manage., 51, 1562-1567, 2001.

Schwede, D., Zhang, L., Vet, R., and Lear, G.: An intercomparison of the deposition models used in CASTNET and CAPMoN networks, Atmos. Environ., 45, 1337-1346, 2011.

Sickles II, J. E. and Shadwick, D. S.: Precision of atmospheric dry deposition data from the Clean Air Status and Trends Network, Atmos. Environ., 36, 5671-5686, 2002.

Sickles II, J. E. and Shadwick, D. S.: Seasonal and regional air quality and atmospheric deposition in the eastern United States, J. Geophys. Res., 112, D17302, doi:10.1029/2006JD008356, 2007a.

Sickles II, J. E. and Shadwick, D. S.: Changes in air quality and atmospheric deposition in the eastern United States: 1990-2004, J. Geophys. Res., 112, D17301, doi:10.1029/2006JD007843, 2007b.

Sickles II, J. E. and Shadwick, D. S.: Comparison of particulate sulfate and nitrate at collocated CASTNET and IMPROVE sites in the eastern US, Atmos. Environ., 42, 2062-2073, 2008.

Sickles II, J. E., Shadwick, D. S., Kilaru, J. V., and Grimm, J. W.: Errors in representing regional acid deposition with spatially sparse monitoring: case studies of the eastern US using model predictions, Atmos. Environ., 43, 2855-2861, 2009.

Sirois, A., Vet, R., and Lamb, D.: A comparison of the precipitation chemistry measurements obtained by the CAPMoN and NADP/NTN networks, Environ. Monit. Assess., 62, 273-303, 2000.

Stanier, C., Singh, A., Adamski, W., Baek, J., Caughey, M., Carmichael, G., Edgerton, E., Kenski, D., Koerber, M., Oleson, J., Rohlf, T., Lee, S. R., Riemer, N., Shaw, S., Sousan, S., and Spak, S. N.: Overview of the LADCO winter nitrate study: hourly ammonia, nitric acid and $\mathrm{PM}_{2.5}$ composition at an urban and rural site pair during $\mathrm{PM}_{2.5}$ episodes in the US Great Lakes region, Atmos. Chem. Phys., 12, 11037-11056, doi:10.5194/acp12-11037-2012, 2012.

Wesely, M. L., Cook, D. R., and Hart, R. L.: Measurements and parameterization of particulate sulfur dry deposition over grass, J. Geophys. Res., 90, 2131-2143, 1985.

West, J. J., Ansari, S., and Pandis, S. N.: Marginal PM 2.5 : nonlinear aerosol mass response to sulfate reductions in the eastern United States, J. Air Waste Manage., 49, 1415-1424, 1999.

Wetherbee, G. A., Shaw, M. J., Latysh, N. E., Lehmann, C. M. B., and Rothert, J. E.: Comparison of precipitation chemistry measurements obtained by the Canadian Air and Precipitation Monitoring Network and National Atmospheric Deposition Program for the period 1995-2004, Environ. Monit. Assess., 164, 111132, doi:10.1007/s10661-009-0879-8, 2010.

Wojcik, G. S. and Chang, J. S.: A re-evaluation of sulfur budgets, lifetimes, and scavenging ratios of eastern North America, J. Atmos. Chem., 26,109-145, 1997.

Wolff, G. T.: On the nature of nitrate in coarse continental aerosols, Atmos. Environ., 18, 977-981, 1984. 
Xing, J., Pleim, J., Mathur, R., Pouliot, G., Hogrefe, C., Gan, C.-M., and Wei, C.: Historical gaseous and primary aerosol emissions in the United States from 1990 to 2010, Atmos. Chem. Phys., 13, 7531-7549, doi:10.5194/acp-13-7531-2013, 2013.

Zhang, L., Vet, R., Wiebe, A., Mihele, C., Sukloff, B., Chan, E., Moran, M. D., and Iqbal, S.: Characterization of the sizesegregated water-soluble inorganic ions at eight Canadian rural sites, Atmos. Chem. Phys., 8, 7133-7151, doi:10.5194/acp-87133-2008, 2008.
Zhang, L., Vet, R., O’Brien, J. M., Mihele, C., Liang, Z., and Wiebe, A.: Dry deposition of individual nitrogen species at eight Canadian rural sites, J. Geophys. Res., 114, D02301, doi:10.1029/2008JD010640, 2009.

Zhang, L., Jacob, D. J., Knipping, E. M., Kumar, N., Munger, J. W., Carouge, C. C., van Donkelaar, A., Wang, Y. X., and Chen, D.: Nitrogen deposition to the United States: distribution, sources, and processes, Atmos. Chem. Phys., 12, 4539-4554, doi:10.5194/acp-12-4539-2012, 2012. 